\title{
Republic of Kazakhstan: Statistical Appendix
}

This Statistical Appendix for the Republic of Kazakhstan was prepared by a staff team of the International Monetary Fund as background documentation for the periodic consultation with the member country. It is based on the information available at the time it was completed on July 6, 2004. The views expressed in this document are those of the staff team and do not necessarily reflect the views of the government of the Republic of Kazakhstan or the Executive Board of the IMF.

The policy of publication of staff reports and other documents by the IMF allows for the deletion of market-sensitive information.

To assist the IMF in evaluating the publication policy, reader comments are invited and may be sent by e-mail to publicationpolicy@imf.org.

Copies of this report are available to the public from

International Monetary Fund • Publication Services

700 19th Street, N.W. • Washington, D.C. 20431

Telephone: (202) $6237430 \bullet$ Telefax: (202) 6237201

E-mail: publications@imf.org • Internet: http://www.imf.org

Price: $\$ 15.00$ a copy

\section{International Monetary Fund \\ Washington, D.C.}





\title{
INTERNATIONAL MONETARY FUND
}

\author{
REPUBLIC OF KAZAKHSTAN
}

\section{Statistical Appendix}

Prepared by a staff team consisting of Julian Berengaut, Katrin Elborgh-Woytek, Turgut Kisinbay, Peter Lohmus (all MCD), and Yongzheng Yang (PDR)

Approved by Middle East and Central Asia Department

July 6, 2004

Contents

Page

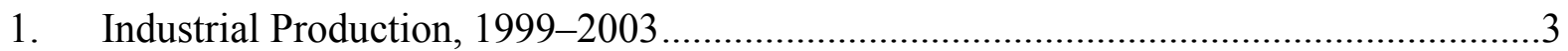

2. Selected Industrial and Agricultural Output Indicators, 1999-2003 ..............................

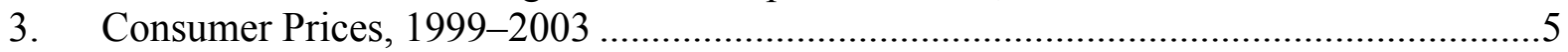

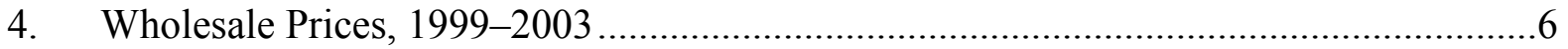

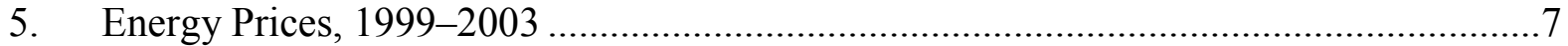

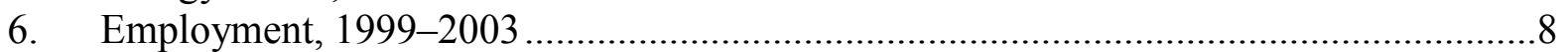

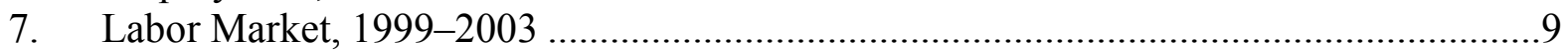

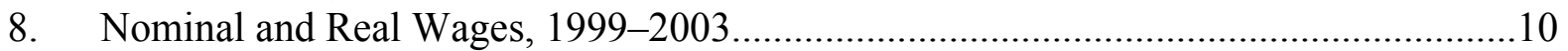

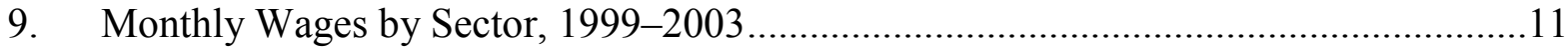

10. Index of Investment in Constant Prices, 1999-2003 ..................................................12

11. Sectoral Composition of Capital Investment in Current Prices, 1999-2003 ...................13

12. Savings Investment Balance, 1999-2003 …………..................................................14

13. Summary Accounts of the National Bank of Kazakhstan, 1999-2003 .........................15

14. Monetary Survey, 1999-2003 ……………….....................................................16

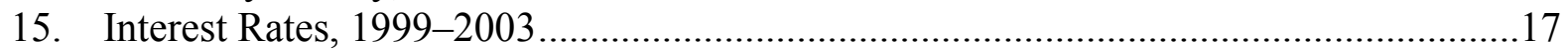

16. Stock Exchange (KASE) Weighted Average Exchange Rates, 1999-2003 ....................18

17. Number of Commercial Banks and Branches, 1999-2003 ..........................................19

18a. General Government Fiscal Operations (in billions of tenge), 1999-2003 ....................20

18b. General Government Fiscal Operations (in percent of GDP), 1999-2003 .....................21

19a. Central Government Fiscal Operations (in billions of tenge), 1999-2003 .....................22

19b. Central Government Fiscal Operations (in percent of GDP), 1999-2003 ......................23

20a. Local Government Fiscal Operations (in billions of tenge), 1999-2003 ........................24

20b. Local Government Fiscal Operations (in percent of GDP), 1999-2003 .......................25

21. General Covernment Expenditure by Functional Classification, 1999-2003 ................26

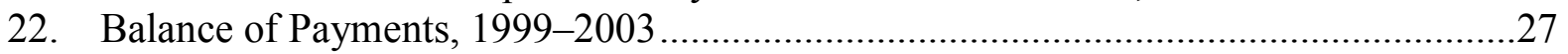

23. Export Volumes, Prices, and Values, 1999-2003 ......................................................28

24. Import Volumes, Prices, and Values, 1999-2003 …………………...........................29 


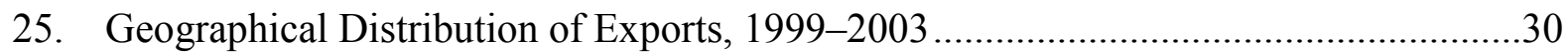

26. Geographical Distribution of Imports, 1999-2003 ....................................................31

27. Geographical Distribution of Exports of Energy Products to the Baltics, Russia, and Other States of the Former Soviet Union, 1999-2003 ….........................................32

28. Breakdown of Foreign Direct Investment by Country of Origin, 1999-2003 _..............33

29. Breakdown of Foreign Direct Investment by Industry, 1999-2003 …........................34

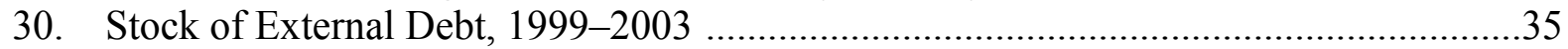


Table 1. Kazakhstan: Industrial Production, 1999-2003 1/

\begin{tabular}{|c|c|c|c|c|c|}
\hline & 1999 & 2000 & 2001 & 2002 & $\begin{array}{r}2003 \\
\text { Preliminary }\end{array}$ \\
\hline & \multicolumn{5}{|c|}{ (In billions of tenge) } \\
\hline Gross output & 1,143 & 1,798 & 2,000 & 2,337 & 2,794 \\
\hline Manufacturing & 578 & 836 & 937 & 1,011 & 1,208 \\
\hline Processing of agricultural products & 181 & 239 & 277 & 251 & 319 \\
\hline Textile and clothing industry & 18 & 36 & 40 & 40 & 49 \\
\hline Manufacturing of coke, refined petroleum products and nuclear fuel & 55 & 70 & 86 & 92 & 128 \\
\hline of which: manufacturing of refined petroleum products & 46 & 60 & 78 & 85 & 123 \\
\hline Chemical industry & 13 & 18 & 24 & 31 & 38 \\
\hline Production of rubber and plastic products & 2 & 4 & 7 & 9 & 12 \\
\hline Manufacture of other non-metal mineral products & 9 & 14 & 24 & 31 & 43 \\
\hline Metallurgy and metal working & 256 & 377 & 374 & 414 & 482 \\
\hline of which: metallurgy & 250 & 366 & 358 & 397 & 459 \\
\hline Machine building & 26 & 46 & 67 & 72 & 89 \\
\hline Other & 17 & 32 & 38 & 71 & 48 \\
\hline Mining & 413 & 800 & 887 & 1,120 & 1,360 \\
\hline Mining of coal and lignite & 22 & 27 & 34 & 33 & 40 \\
\hline Extraction of crude petroleum and condensate gas & 333 & 683 & 721 & 923 & 1,133 \\
\hline Extraction of natural gas & 7 & 9 & 13 & 9 & 13 \\
\hline Mining of metal ores & 37 & 55 & 66 & 82 & 99 \\
\hline Other mining & 15 & 26 & 52 & 73 & 75 \\
\hline \multirow[t]{2}{*}{ Production and distribution of electricity, gas, and water } & 152 & 162 & 177 & 205 & 226 \\
\hline & \multicolumn{5}{|c|}{ (In percent of total) } \\
\hline \multicolumn{6}{|l|}{ Total } \\
\hline Manufacturing & 50.6 & 46.5 & 46.9 & 43.3 & 43.2 \\
\hline Processing of agricultural products & 15.8 & 13.3 & 13.9 & 10.7 & 11.4 \\
\hline Textile and clothing industry & 1.6 & 2.0 & 2.0 & 1.7 & 1.8 \\
\hline Manufacturing of coke, refined petroleum products and nuclear fuel & 4.8 & 3.9 & 4.3 & 3.9 & 4.6 \\
\hline of which: manufacturing of refined petroleum products & 4.0 & 3.3 & 3.9 & 3.6 & 4.4 \\
\hline Chemical industry & 1.2 & 1.0 & 1.2 & 1.3 & 1.4 \\
\hline Production of rubber and plastic products & 0.2 & 0.2 & 0.3 & 0.4 & 0.4 \\
\hline Manufacture of other non-metal mineral products & 0.8 & 0.8 & 1.2 & 1.3 & 1.5 \\
\hline Metallurgy and metal working & 22.4 & 20.9 & 18.7 & 17.7 & 17.3 \\
\hline of which: metallurgy & 21.8 & 20.3 & 17.9 & 17.0 & 16.4 \\
\hline Machine building & 2.2 & 2.5 & 3.4 & 3.1 & 3.2 \\
\hline Other & 1.5 & 1.8 & 1.9 & 3.0 & 1.7 \\
\hline Mining & 36.1 & 44.5 & 44.3 & 47.9 & 48.7 \\
\hline Mining of coal and lignite & 1.9 & 1.5 & 1.7 & 1.4 & 1.4 \\
\hline Extraction of crude petroleum and condensate gas & 29.1 & 38.0 & 36.1 & 39.5 & 40.6 \\
\hline Extraction of natural gas & 0.6 & 0.5 & 0.6 & 0.4 & 0.5 \\
\hline Mining of metal ores & 3.2 & 3.0 & 3.3 & 3.5 & 3.5 \\
\hline Other mining & 1.3 & 1.5 & 2.6 & 3.1 & 2.7 \\
\hline Production and distribution of electricity, gas, and water & 13.3 & 9.0 & 8.8 & 8.8 & 8.1 \\
\hline
\end{tabular}

Source: National Statistical Agency.

1/ The values do not include intrafactory turnover. 
Table 2. Kazakhstan: Selected Industrial and Agricultural Output Indicators, 1999-2003

\begin{tabular}{|c|c|c|c|c|c|}
\hline & 1999 & 2000 & 2001 & 2002 & $\begin{array}{l}2003 \\
\text { iminary }\end{array}$ \\
\hline \multicolumn{6}{|l|}{ Industry } \\
\hline \multicolumn{6}{|l|}{ Production volumes } \\
\hline Crude oil (in thousands of metric tons) $1 /$ & 30,130 & 35,317 & 40,091 & 47,271 & 51,390 \\
\hline Coal (in thousands of metric tons) & 58,378 & 74,872 & 79,135 & 73,731 & 84,717 \\
\hline Natural gas (in millions of cubic meters) & 9,946 & 11,542 & 11,610 & 14,109 & 16,221 \\
\hline Iron ore (in thousands of metric tons) & 9,617 & 16,157 & 15,886 & 17,675 & 19,365 \\
\hline Electricity (in millions of kwh) & 47,497 & 51,635 & 55,384 & 58,331 & 63,667 \\
\hline & \multicolumn{5}{|c|}{ (Percentage change over previous year) } \\
\hline \multicolumn{6}{|l|}{ Production volume growth } \\
\hline Crude oil (in thousands of metric tons) 1/ & 16.1 & 17.2 & 13.5 & 17.9 & 8.7 \\
\hline Coal (in thousands of metric tons) & -16.3 & 28.3 & 5.7 & -6.8 & 14.9 \\
\hline Natural gas (in millions of cubic meters) & 25.1 & 16.0 & 0.6 & 21.5 & 15.0 \\
\hline Iron ore (in thousands of metric tons) & 3.0 & 68.0 & -1.7 & 11.3 & 9.6 \\
\hline Electricity (in millions of kwh) & -3.4 & 8.7 & 7.3 & 5.3 & 9.1 \\
\hline \multicolumn{6}{|l|}{ Agriculture } \\
\hline \multicolumn{6}{|l|}{ Production volumes } \\
\hline Meat (in thousands of metric tons) & 1,182 & 1,140 & 1,155 & 1,191 & 1,241 \\
\hline Cereals (in thousands of metric tons) & 14,264 & 11,565 & 15,897 & 15,960 & 14,777 \\
\hline of which: Wheat & 11,242 & 9,073 & 12,707 & 12,700 & 11,537 \\
\hline & \multicolumn{5}{|c|}{ (Percentage change over previous year) } \\
\hline \multicolumn{6}{|l|}{ Production volume growth } \\
\hline Processed meat (in thousands of tons) & -13.5 & -14.4 & -3.8 & -9.4 & 19.4 \\
\hline Milk products (in thousands of tons) & -18.9 & 22.2 & -1.0 & 12.9 & 14.7 \\
\hline Cereals & 123.0 & -19.0 & 37.5 & 0.4 & -7.4 \\
\hline of which: Wheat & 136.9 & -19.3 & 40.1 & -0.1 & -9.2 \\
\hline Potatoes & 34.2 & 0.2 & 29.1 & 3.8 & 1.7 \\
\hline Tobacco & -8.3 & 102.0 & -8.8 & 7.5 & 0.4 \\
\hline Vegetables & 19.3 & 19.9 & 15.4 & 4.2 & 4.4 \\
\hline Meat & -2.5 & -3.6 & 1.3 & 3.1 & 4.2 \\
\hline Milk & 5.1 & 5.5 & 5.2 & 4.8 & 5.2 \\
\hline Eggs & 8.4 & 11.9 & 9.6 & 13.3 & 8.5 \\
\hline Wool & -11.7 & 2.9 & 3.1 & 5.1 & 8.5 \\
\hline & \multicolumn{5}{|c|}{ (In billions of tenge) } \\
\hline \multicolumn{6}{|l|}{ Production value } \\
\hline $\begin{array}{c}\text { Plant growing } \\
\text { of which: }\end{array}$ & 177.7 & 223.5 & 325.8 & 325.3 & 348.6 \\
\hline Cereals & 85.8 & 111.5 & 187.4 & 165.9 & 175.5 \\
\hline of which: Wheat & 67.9 & 89.0 & 143.9 & 123.0 & 139.2 \\
\hline Potatoes & 26.3 & 31.2 & 36.0 & 35.6 & 41.9 \\
\hline Vegetables & 25.2 & 26.9 & 41.6 & 43.2 & 36.7 \\
\hline \multirow[t]{2}{*}{ Animal husbandry } & 157.3 & 178.5 & 207.9 & 232.1 & 258.1 \\
\hline & \multicolumn{5}{|c|}{ (In percent of total production) } \\
\hline \multicolumn{6}{|l|}{ Share of agricultural production by private farms } \\
\hline Meat & 91.4 & 93.7 & 93.9 & 94.0 & 94.0 \\
\hline Milk & 94.8 & 95.0 & 95.4 & 95.5 & 95.6 \\
\hline Eggs & 47.6 & 49.8 & 50.0 & 46.8 & 46.3 \\
\hline Wool & 87.3 & 89.1 & 91.1 & 91.6 & 92.7 \\
\hline Potatoes & 94.9 & 95.8 & 96.0 & 96.9 & 96.7 \\
\hline Vegetables & 88.6 & 94.0 & 93.6 & 96.2 & 96.5 \\
\hline
\end{tabular}

Source: National Statistical Agency.

$1 /$ Includes gas condensate. 
Table 3. Kazakhstan: Consumer Prices, 1999-2003

\begin{tabular}{|c|c|c|c|c|c|c|c|c|c|c|c|c|c|}
\hline & & Jan. & Feb. & Mar. & Apr. & May & Jun. & Jul. & Aug. & Sep. & Oct. & Nov. & Dec. \\
\hline & & \multicolumn{12}{|c|}{ (Percentage change over previous month) } \\
\hline \multirow[t]{9}{*}{1999} & Total & 0.9 & -0.2 & -0.2 & 4.6 & 1.4 & 4.8 & 1.1 & -0.3 & 0.7 & 0.7 & 1.7 & 1.7 \\
\hline & Food & 1.0 & -0.3 & -0.3 & 5.7 & 1.7 & 6.6 & 0.6 & -1.2 & 0.3 & 0.6 & 2.1 & 2.7 \\
\hline & Clothing and footwear & 0.2 & 0.1 & 0.0 & 3.5 & 0.6 & 2.0 & 0.6 & 0.6 & 1.5 & 1.5 & 1.3 & 0.9 \\
\hline & Rent, water, and power & 2.1 & -0.1 & -0.1 & 0.3 & 1.2 & 0.7 & 1.6 & 0.4 & -0.2 & 0.0 & 1.0 & 0.3 \\
\hline & Household goods & 0.0 & -0.1 & -0.3 & 9.9 & 1.7 & 3.7 & 0.9 & 0.5 & 0.6 & 1.8 & 0.7 & 0.6 \\
\hline & Medical care & -0.5 & -1.2 & -0.9 & 6.4 & -0.2 & 0.9 & 0.4 & 0.2 & 0.6 & 1.1 & 0.7 & 0.5 \\
\hline & Transport and communication & 0.0 & -0.8 & -0.5 & 3.5 & 2.4 & 8.6 & 4.3 & 1.8 & 3.0 & 0.0 & 2.0 & 0.3 \\
\hline & Recreation, education, and culture & 0.3 & 1.2 & 0.4 & 6.6 & 0.7 & 2.8 & 1.1 & 0.9 & 1.8 & 1.2 & 0.7 & 0.7 \\
\hline & Personal care & 0.1 & 0.3 & 0.4 & 11.7 & 2.5 & 3.1 & 1.7 & 0.6 & 0.6 & 1.4 & 1.0 & 0.7 \\
\hline \multirow[t]{9}{*}{2000} & Total & 2.6 & 0.1 & 0.0 & 0.4 & 0.7 & 0.7 & 0.4 & 0.2 & 0.5 & 1.2 & 1.5 & 1.3 \\
\hline & Food & 3.5 & 0.2 & -0.1 & 0.3 & 1.1 & 1.0 & 0.1 & 0.0 & 0.2 & 1.6 & 2.1 & 2.0 \\
\hline & Clothing and footwear & 0.6 & 0.4 & 0.3 & 0.4 & 0.5 & 0.4 & 0.6 & 0.4 & 0.5 & 1.0 & 1.0 & 0.7 \\
\hline & Rent, water, and power & 3.8 & -0.6 & 0.1 & 0.1 & 0.1 & 0.1 & 0.2 & 0.2 & 1.7 & 1.1 & 0.7 & 0.2 \\
\hline & Household goods & 0.4 & 0.1 & -0.3 & -0.1 & 0.5 & 0.3 & 0.2 & 0.2 & 0.2 & 0.2 & 0.5 & 0.6 \\
\hline & Medical care & 0.7 & 0.5 & 0.9 & 0.7 & 0.2 & 0.4 & 0.3 & 0.1 & 0.2 & 0.1 & 0.4 & 0.2 \\
\hline & Transport and communication & -0.1 & -0.1 & -0.9 & 1.4 & 0.6 & 0.7 & 2.5 & 1.1 & 0.2 & 0.7 & 0.9 & 0.3 \\
\hline & Recreation, education, and culture & 0.7 & 0.3 & 0.4 & 0.2 & 0.1 & 0.1 & 0.4 & 0.2 & 0.3 & 0.4 & 0.4 & 0.7 \\
\hline & Personal care & 0.7 & 0.4 & 0.1 & 0.2 & 0.4 & 0.7 & 0.3 & 0.3 & 0.2 & 0.8 & 0.4 & 0.2 \\
\hline \multirow[t]{9}{*}{2001} & Total & 1.1 & 0.7 & 0.7 & 0.7 & 0.4 & 0.1 & -0.1 & 0.0 & 0.2 & 0.7 & 0.9 & 1.0 \\
\hline & Food & 1.2 & 1.4 & 1.3 & 1.2 & 0.7 & 0.0 & -0.4 & -0.6 & 0.1 & 0.6 & 1.3 & 1.7 \\
\hline & Clothing and footwear & 0.4 & 0.4 & 0.6 & 0.5 & 0.5 & 0.4 & 0.3 & 0.3 & 0.6 & 1.1 & 1.2 & 0.8 \\
\hline & Rent, water, and power & 2.0 & -0.6 & 0.1 & 0.1 & 0.1 & 0.0 & -0.4 & 0.8 & 0.1 & 1.0 & 0.3 & 0.3 \\
\hline & Household goods & 0.4 & 0.6 & 0.3 & 0.3 & 0.4 & 0.2 & 0.4 & 0.2 & 0.3 & 0.2 & 0.9 & 0.5 \\
\hline & Medical care & 0.6 & 0.3 & 0.1 & -0.1 & -0.1 & 0.2 & 0.2 & 0.3 & -0.2 & 0.2 & 0.1 & 0.2 \\
\hline & Transport and communication & 0.0 & -0.6 & -0.6 & -0.3 & -0.3 & 0.3 & 1.0 & 1.0 & 0.5 & 0.2 & -0.5 & -1.3 \\
\hline & Recreation, education, and culture & 0.3 & 0.5 & 0.4 & 0.3 & 0.1 & 0.1 & 0.3 & 0.2 & 0.3 & 0.4 & 0.5 & 0.4 \\
\hline & Personal care & 0.4 & 0.3 & 0.3 & 0.5 & 0.2 & 0.4 & 0.4 & 0.3 & 0.4 & 0.5 & 0.6 & 0.5 \\
\hline \multirow[t]{12}{*}{2002} & Total & 0.7 & 0.3 & 0.2 & 0.5 & 0.9 & 0.5 & 0.6 & -0.3 & 0.1 & 0.6 & 1.0 & 1.4 \\
\hline & Food & 1.1 & 0.5 & 0.2 & 0.4 & 0.7 & 0.2 & 0.6 & -0.6 & -0.1 & 0.3 & 1.2 & 2.3 \\
\hline & Clothing and footwear & 0.3 & 0.3 & 0.5 & 0.6 & 0.6 & 0.4 & 0.3 & 0.5 & 0.7 & 1.2 & 1.1 & 1.0 \\
\hline & Rent, water, and power & 1.1 & 0.6 & 0.2 & 0.9 & 0.0 & 0.3 & 1.1 & -0.1 & 0.2 & 1.0 & 0.6 & 0.3 \\
\hline & Household goods & 0.2 & 0.5 & 0.4 & 0.4 & 0.3 & 0.4 & 0.4 & 0.2 & 0.3 & 0.4 & 0.5 & 0.4 \\
\hline & Medical care & 0.2 & 1.0 & 0.4 & 0.6 & 0.4 & 0.5 & 0.6 & 0.5 & 0.2 & 0.6 & 0.5 & 0.4 \\
\hline & Transport & -2.2 & -2.8 & -2.0 & 0.1 & 7.8 & 3.7 & 0.0 & -0.5 & -1.1 & 0.7 & 0.6 & 0.7 \\
\hline & Communication & 0.1 & 0.1 & 0.1 & 1.0 & 0.0 & 0.0 & 0.0 & 0.0 & 0.0 & 0.0 & 0.0 & 0.0 \\
\hline & Rest, recreation, and culture & 0.3 & 0.7 & 0.4 & 0.4 & 0.3 & 0.1 & 0.2 & 0.3 & 0.5 & 0.5 & 0.5 & 0.6 \\
\hline & Education & 0.2 & 0.6 & 0.8 & 0.2 & 0.2 & 0.1 & 0.3 & 0.1 & 3.0 & 1.2 & 1.4 & 0.3 \\
\hline & Restaurants and hotels & 0.3 & 0.7 & 0.6 & 0.3 & 0.6 & 0.1 & 0.1 & 0.2 & 0.3 & 0.3 & 0.6 & 0.8 \\
\hline & Other goods and services & 0.5 & 0.6 & 0.4 & 0.5 & 0.4 & 0.5 & 0.4 & 0.4 & 0.5 & 0.4 & 0.3 & 0.3 \\
\hline \multirow[t]{13}{*}{2003} & Total & 1.0 & 0.5 & 0.3 & 0.4 & 0.1 & 0.1 & 0.0 & 0.2 & 0.4 & 1.3 & 1.6 & 0.9 \\
\hline & Food & 1.4 & 0.8 & 0.2 & 0.4 & 0.1 & 0.0 & -0.5 & -0.5 & 0.0 & 1.7 & 1.9 & 1.5 \\
\hline & Clothing and footwear & 0.7 & 0.6 & 0.5 & 0.5 & 0.6 & 0.4 & 0.3 & 0.4 & 0.5 & 0.6 & 0.7 & 0.7 \\
\hline & Rent, water, and power & 0.6 & 0.1 & 0.2 & 0.9 & 0.0 & 0.0 & 0.0 & 0.2 & 0.1 & 1.8 & 0.7 & 0.3 \\
\hline & Household goods & 0.4 & 0.5 & 0.4 & 0.4 & 0.2 & 0.4 & 0.2 & 0.3 & 0.3 & 0.3 & 0.3 & 0.4 \\
\hline & Medical care & 0.5 & 0.6 & 0.3 & 0.1 & 0.3 & 0.6 & 0.1 & 0.1 & 0.1 & 0.0 & 0.2 & 0.4 \\
\hline & Transport & 0.0 & -1.0 & 0.3 & -0.7 & -1.4 & -0.2 & 2.5 & 4.7 & 2.9 & 1.0 & 4.3 & -0.3 \\
\hline & Communication & 0.2 & 0.0 & 0.0 & 0.9 & 0.0 & 0.0 & 0.0 & 0.0 & 0.0 & 0.1 & 0.0 & 0.0 \\
\hline & Rest, recreation, and culture & 0.7 & 0.5 & 0.4 & 0.2 & 0.1 & 0.1 & 0.1 & 0.2 & 0.3 & 0.3 & 0.4 & 0.4 \\
\hline & Education & 0.4 & 0.2 & 0.3 & 0.2 & 0.1 & 0.2 & 0.2 & 0.2 & 4.6 & 1.5 & 0.6 & 0.2 \\
\hline & Restaurants and hotels & 0.6 & 0.5 & 0.6 & 0.2 & 0.3 & 0.3 & 0.4 & 0.5 & 0.2 & 0.5 & 1.3 & 0.7 \\
\hline & Other goods and services & 0.7 & 0.4 & 0.4 & 0.1 & 0.4 & 0.4 & 0.4 & 0.5 & 0.4 & 0.2 & 0.5 & 0.3 \\
\hline & & \multicolumn{12}{|c|}{ (Percentage change over previous year) } \\
\hline \multicolumn{14}{|c|}{ Memorandum items: } \\
\hline & Total 1999 & 1.0 & -0.3 & -1.2 & 2.8 & 3.9 & 9.8 & 11.2 & 11.9 & 12.8 & 14.3 & 16.3 & 17.8 \\
\hline & Total 2000 & 19.8 & 20.2 & 20.4 & 15.6 & 14.7 & 10.2 & 9.5 & 10.0 & 9.8 & 10.4 & 10.2 & 9.8 \\
\hline & Total 2001 & 8.2 & 8.8 & 9.6 & 10.0 & 9.7 & 9.0 & 8.5 & 8.3 & 8.0 & 7.4 & 6.8 & 6.4 \\
\hline & Total 2002 & 6.0 & 5.7 & 5.0 & 4.8 & 5.4 & 5.8 & 6.5 & 6.2 & 6.1 & 6.0 & 6.1 & 6.6 \\
\hline & Total 2003 & 6.9 & 7.0 & 7.2 & 7.0 & 6.1 & 5.7 & 5.1 & 5.6 & 5.9 & 6.7 & 7.3 & 6.8 \\
\hline
\end{tabular}

Source: National Statistical Agency. 
Table 4. Kazakhstan: Wholesale Prices, 1999-2003

\begin{tabular}{|c|c|c|c|c|c|c|c|c|c|c|c|c|c|}
\hline & & Jan. & Feb. & Mar. & Apr. & May & Jun. & Jul. & Aug. & Sep. & Oct. & Nov. & Dec. \\
\hline & & \multicolumn{12}{|c|}{ (Percentage change over previous month) } \\
\hline \multirow{12}{*}{1999} & Total & & & & & & & & & & & & \\
\hline & Mining and extraction industry & -1.7 & -2.9 & 1.2 & 9.7 & 10.9 & 13.0 & 2.2 & 8.1 & 6.9 & 13.5 & 4.1 & 5.4 \\
\hline & Extraction of crude oil and natural gas & -3.2 & -6.8 & 3.1 & 17.9 & 11.7 & 21.4 & 2.9 & 11.8 & 11.6 & 19.6 & 6.1 & 8.5 \\
\hline & Processing industry & -0.9 & -1.1 & -1.2 & 9.4 & 9.2 & 7.1 & 4.3 & 2.6 & 3.2 & 7.5 & 3.7 & 4.1 \\
\hline & Processing of agricultural products & -0.3 & -1.0 & 0.2 & 3.9 & 3.1 & 3.7 & 4.5 & -0.1 & 2.6 & 1.0 & 1.0 & 0.3 \\
\hline & Textile and sewing industry & -0.1 & -0.3 & -2.3 & 1.4 & 0.6 & 0.5 & 0.3 & 0.6 & 2.3 & 0.0 & 2.9 & 0.0 \\
\hline & Coal production, oil refinery & -0.7 & 0.1 & -9.5 & 9.4 & 3.3 & 0.9 & 7.1 & 10.3 & 12.9 & 29.0 & 12.7 & 10.5 \\
\hline & Chemical industry & 0.2 & 0.3 & -1.0 & 6.4 & 3.4 & 0.3 & 4.3 & 0.2 & -2.4 & -1.6 & -1.5 & -0.1 \\
\hline & Manufacturing of rubber and plastic products & 0.0 & 0.0 & 0.0 & 0.5 & 0.1 & 0.2 & 0.0 & 0.0 & 0.1 & 0.1 & 0.0 & 0.0 \\
\hline & Metallurgical industry and metal working & -1.9 & -2.3 & 1.3 & 16.0 & 18.1 & 13.0 & 4.1 & 2.3 & 1.3 & 5.2 & 1.8 & 3.7 \\
\hline & Manufacturing of machinery and equipment & 0.3 & 0.9 & 0.6 & 4.3 & -0.1 & 1.4 & 0.0 & -0.2 & 0.1 & 0.2 & 0.5 & 0.4 \\
\hline & Electricity, gas, and water supply & -0.1 & 0.2 & 0.3 & 0.0 & 0.9 & 0.6 & 1.3 & 0.3 & -0.1 & 0.1 & 0.1 & 0.0 \\
\hline \multirow[t]{12}{*}{2000} & Total & 0.9 & 2.0 & 2.2 & -1.2 & -2.2 & 3.1 & 1.7 & 1.8 & 3.9 & 2.2 & 0.9 & 2.7 \\
\hline & Mining and extraction industry & 1.7 & 5.5 & 5.8 & -2.8 & -9.0 & 11.2 & 3.8 & 1.1 & 10.0 & 4.8 & 2.3 & 5.1 \\
\hline & Extraction of crude oil and natural gas & 1.7 & 6.2 & 7.8 & -3.4 & -11.1 & 14.8 & 4.8 & 1.2 & 12.9 & 5.6 & 2.6 & 5.7 \\
\hline & Processing industry & 1.0 & 0.4 & 0.5 & -0.6 & 1.7 & -0.8 & 0.8 & 3.0 & 1.1 & 0.7 & 0.0 & 1.6 \\
\hline & Processing of agricultural products & 0.1 & 0.0 & -0.1 & -0.1 & 3.6 & 1.4 & 1.0 & -0.5 & 0.8 & 1.0 & 0.7 & 1.1 \\
\hline & Textile and sewing industry & 0.5 & 0.2 & 0.2 & 0.2 & 0.8 & 0.3 & 0.1 & 0.7 & 0.4 & 0.0 & 0.5 & 1.0 \\
\hline & Coal production, oil refinery & -1.8 & -6.2 & -2.9 & 0.0 & 2.5 & 0.1 & 1.7 & 20.8 & 3.0 & 5.1 & 5.6 & 4.6 \\
\hline & Chemical industry & 3.1 & 1.4 & -0.9 & 1.9 & 3.7 & 0.4 & -0.7 & -2.4 & 0.2 & 0.0 & 0.7 & -0.7 \\
\hline & Manufacturing of rubber and plastic products & 0.0 & 4.9 & 6.1 & 0.0 & -4.6 & 0.0 & 0.3 & 0.1 & 0.0 & 0.0 & 0.1 & 0.2 \\
\hline & Metallurgical industry and metal working & 2.3 & 2.4 & 1.8 & -1.4 & 0.9 & -2.5 & 0.7 & 1.7 & 0.9 & -0.6 & -2.4 & 1.5 \\
\hline & Manufacturing of machinery and equipment & 0.8 & 0.2 & -0.8 & 1.0 & -0.2 & -0.4 & 0.0 & -0.3 & 0.5 & -0.2 & 1.9 & 0.1 \\
\hline & Electricity, gas, and water supply & -0.5 & 0.2 & 0.0 & 0.4 & 0.0 & 0.0 & 0.3 & 0.1 & 0.0 & 1.0 & 0.1 & 0.0 \\
\hline \multirow[t]{12}{*}{2001} & Total & -8.5 & 2.0 & 1.3 & -3.7 & 0.0 & 1.1 & -0.3 & -2.0 & 0.5 & 0.1 & -1.6 & -3.4 \\
\hline & Mining and extraction industry & -18.0 & 5.4 & 3.0 & -7.8 & 2.4 & 3.5 & -1.9 & -2.8 & 1.9 & -1.0 & -3.3 & -5.9 \\
\hline & Extraction of crude oil and natural gas & -20.4 & 5.9 & 3.5 & -9.0 & 2.5 & 4.2 & -2.2 & -3.4 & 1.8 & -1.7 & -4.1 & -7.1 \\
\hline & Processing industry & -1.2 & -0.3 & 0.0 & -1.0 & -2.0 & -0.7 & 0.4 & -1.9 & -0.6 & 0.9 & -0.8 & -2.2 \\
\hline & Processing of agricultural products & 0.4 & 0.8 & 1.1 & 0.3 & 0.5 & 1.0 & 0.5 & -0.1 & -0.2 & 0.0 & 0.6 & -0.1 \\
\hline & Textile and sewing industry & 0.3 & 0.2 & 0.2 & 0.2 & 0.0 & 0.4 & 0.3 & 0.0 & 0.2 & 1.7 & 0.3 & 0.1 \\
\hline & Coal production, oil refinery & -1.8 & -3.6 & -4.3 & -4.5 & -2.5 & -0.2 & 8.1 & 0.2 & 1.1 & 2.8 & -0.8 & -16.2 \\
\hline & Chemical industry & 0.6 & -0.8 & 1.2 & 2.0 & -0.8 & -1.2 & 1.3 & 1.5 & 1.2 & 0.3 & -0.3 & 0.1 \\
\hline & Manufacturing of rubber and plastic products & 0.1 & 0.3 & 0.1 & 0.1 & 0.1 & 1.3 & 0.4 & 0.1 & 0.2 & 0.1 & 0.1 & 0.3 \\
\hline & Metallurgical industry and metal working & -1.9 & 0.1 & 0.6 & -0.9 & -3.2 & -1.7 & -1.7 & -4.0 & -1.6 & 0.8 & -1.7 & 0.7 \\
\hline & Manufacturing of machinery and equipment & -0.1 & 0.6 & 0.7 & -0.8 & 0.9 & 0.4 & 1.3 & 0.1 & -0.3 & 0.1 & 0.0 & -0.1 \\
\hline & Electricity, gas, and water supply & 1.0 & -0.1 & 0.0 & 0.6 & 0.0 & 0.0 & 2.7 & 0.0 & 0.1 & 0.5 & 0.9 & 0.3 \\
\hline \multirow[t]{12}{*}{2002} & Total & -4.6 & 0.6 & 2.1 & 3.9 & 3.5 & 0.2 & 2.5 & 1.7 & 2.1 & 2.4 & -1.6 & -1.1 \\
\hline & Mining and extraction industry & -9.2 & 1.1 & 2.2 & 8.2 & 6.5 & 0.1 & 3.7 & 3.3 & 4.8 & 4.8 & -2.8 & -3.0 \\
\hline & Extraction of crude oil and natural gas & -11.0 & 1.0 & 2.3 & 9.1 & 7.3 & 0.4 & 4.1 & 3.6 & 5.9 & 5.6 & -3.2 & -3.5 \\
\hline & Processing industry & -1.7 & 0.3 & 2.5 & 0.5 & 1.1 & 0.4 & 1.5 & 0.3 & -0.4 & 0.0 & -0.5 & 1.0 \\
\hline & Processing of agricultural products & 0.3 & 0.4 & -0.3 & -0.1 & -0.5 & -0.5 & -0.3 & -0.2 & -0.1 & -0.3 & -0.3 & 0.1 \\
\hline & Textile and sewing industry & 0.9 & 2.1 & 2.1 & 0.0 & 0.1 & -0.1 & 0.0 & 0.0 & 0.4 & 0.4 & 2.1 & 0.0 \\
\hline & Coal production, oil refinery & -3.4 & -5.7 & 0.0 & -0.3 & -0.2 & 0.8 & -0.1 & 2.1 & 0.6 & 0.4 & -0.2 & -0.1 \\
\hline & Chemical industry & 0.8 & 0.6 & -1.0 & 1.2 & 1.6 & -1.0 & 0.4 & 0.5 & 0.4 & -0.3 & -0.2 & 0.3 \\
\hline & Manufacturing of rubber and plastic products & 0.2 & 0.7 & 0.7 & 0.7 & 0.1 & 0.5 & 0.0 & -0.2 & 0.2 & 0.0 & -0.1 & 2.2 \\
\hline & Metallurgical industry and metal working & -2.5 & 1.8 & 4.6 & 1.1 & 2.2 & 0.7 & 2.8 & 0.1 & -0.9 & 0.0 & -0.8 & 1.8 \\
\hline & Manufacturing of machinery and equipment & 0.3 & 0.5 & 0.2 & -1.4 & 0.3 & 0.1 & -0.1 & 0.0 & -0.2 & 0.0 & 0.1 & -0.3 \\
\hline & Electricity, gas, and water supply & 2.2 & 0.4 & 0.3 & 0.6 & 0.4 & 0.1 & 1.5 & 0.0 & 0.0 & 0.4 & 0.1 & 0.0 \\
\hline \multirow[t]{13}{*}{2003} & Total & & & & & & & & & & & & \\
\hline & Mining and extraction industry & 5.6 & 2.8 & 3.0 & -6.7 & -5.5 & -4.6 & 2.9 & 2.8 & 2.9 & -1.2 & 2.5 & -0.8 \\
\hline & Extraction of crude oil and natural gas & 7.3 & 3.2 & 3.6 & -8.6 & -6.9 & -5.9 & 3.6 & 3.3 & 3.3 & -1.9 & 2.8 & -0.9 \\
\hline & Processing industry & 2.1 & 1.4 & 0.3 & 1.9 & -1.0 & -1.5 & 0.8 & 0.7 & 0.5 & 1.7 & 1.5 & 1.3 \\
\hline & Processing of agricultural products & 0.1 & -0.5 & -0.1 & -0.1 & 0.4 & 0.5 & 0.5 & 0.3 & 0.7 & 1.4 & 1.5 & 1.3 \\
\hline & Textile and sewing industry & 0.1 & 0.1 & 0.2 & 0.0 & 0.2 & 0.0 & 0.0 & 0.2 & 0.1 & 0.4 & 16.6 & 0.0 \\
\hline & Coal production, oil refinery & 1.5 & 2.2 & -0.3 & -0.8 & -1.5 & 0.0 & -0.4 & 0.5 & -0.2 & -0.6 & 1.0 & -0.2 \\
\hline & Chemical industry & 0.2 & -0.4 & -0.6 & -0.4 & 0.3 & 1.2 & -0.2 & -0.1 & 0.4 & 0.4 & 0.6 & -0.3 \\
\hline & Manufacturing of rubber and plastic products & 0.2 & 0.0 & 0.6 & 1.1 & 0.0 & 0.0 & 0.0 & -0.8 & -0.2 & 0.2 & 0.4 & 0.2 \\
\hline & Metallurgical industry and metal working & 4.1 & 2.8 & 0.7 & 4.0 & -2.2 & -3.5 & 1.5 & 1.2 & 0.9 & 2.9 & 1.1 & 2.0 \\
\hline & Manufacturing of machinery and equipment & 0.0 & -0.3 & 0.0 & 1.9 & 0.0 & 0.0 & -0.2 & 0.0 & -1.6 & 0.4 & 1.0 & 1.4 \\
\hline & Electricity, gas, and water supply & 0.9 & 0.3 & 0.0 & 0.7 & 0.0 & -0.3 & -0.9 & 0.1 & 0.0 & 1.8 & 0.4 & 0.0 \\
\hline & & \multicolumn{12}{|c|}{ (Percentage change over previous year) } \\
\hline \multicolumn{14}{|c|}{ Memorandum items: } \\
\hline & Total 1999 & -6.5 & -8.5 & -8.4 & -1.3 & 7.2 & 16.4 & 20.9 & 26.9 & 32.8 & 44.5 & 51.2 & 57.2 \\
\hline & Total 2000 & 60.2 & 65.5 & 69.3 & 56.0 & 41.4 & 35.5 & 33.7 & 31.0 & 31.1 & 23.7 & 20.9 & 19.4 \\
\hline & Total 2001 & 8.3 & 8.3 & 7.3 & 4.6 & 6.9 & 4.9 & 2.8 & -1.1 & -4.3 & -6.4 & -8.7 & -14.1 \\
\hline & Total 2002 & -10.4 & -11.6 & -10.9 & -3.9 & -0.6 & -1.5 & 1.3 & 5.1 & 6.8 & 9.3 & 9.3 & 11.9 \\
\hline & Total 2003 & 21.5 & 23.1 & 22.5 & 15.1 & 8.0 & 4.8 & 3.8 & 3.7 & 3.1 & 1.1 & 4.6 & 5.9 \\
\hline
\end{tabular}

Source: National Statistical Agency. 
Table 5. Kazakhstan: Energy Prices, 1999-2003 1/

\begin{tabular}{|c|c|c|c|c|c|c|c|c|c|c|c|c|}
\hline & Jan. & Feb. & Mar. & Apr. & May & Jun. & Jul. & Aug. & Sep. & Oct. & Nov. & Dec. \\
\hline \multicolumn{13}{|l|}{1999} \\
\hline Crude oil & 3,756 & 3,538 & 3,697 & 4,770 & 5,629 & 7,020 & 7,691 & 8,845 & 10,163 & 12,409 & 13,290 & 14,553 \\
\hline Natural gas & 818 & 821 & 828 & 649 & 656 & 660 & 677 & 676 & 683 & 691 & 726 & 751 \\
\hline Electricity & 2,380 & 2,380 & 2,390 & 2,390 & 2,400 & 2,410 & 2,430 & 2,430 & 2,430 & 2,440 & 2,440 & 2,440 \\
\hline Coal & 468 & 489 & 484 & 485 & 518 & 517 & 516 & 530 & 518 & 512 & 512 & 514 \\
\hline Gasoline & 12,584 & 12,648 & 10,000 & 12,221 & 12,840 & 12,840 & 16,602 & 19,098 & 22,949 & 27,385 & 29,583 & 31,186 \\
\hline Diesel & 9,398 & 9,492 & 8,932 & 10,049 & 10,310 & 10,516 & 9,948 & 11,089 & 11,921 & 14,639 & 16,293 & 20,497 \\
\hline Mazuth & 3,086 & 3,095 & 3,080 & 3,182 & 3,235 & 3,235 & 2,984 & 3,293 & 4,102 & 6,254 & 7,512 & 7,579 \\
\hline Heating (Gcal) & 1,138 & 1,138 & 1,139 & 1,134 & 1,138 & 1,142 & 1,160 & 1,163 & 1,163 & 1,161 & 1,159 & 1,159 \\
\hline Liquid petroleum gas (mt) & 4,117 & 3,592 & 3,494 & 3,891 & 3,945 & 4,502 & 3,928 & 3,134 & 3,335 & 3,409 & 4,688 & 4,796 \\
\hline \multicolumn{13}{|l|}{2000} \\
\hline Crude oil & 14,862 & 15,268 & 16,327 & 15,729 & 13,642 & 15,941 & 16,763 & 16,552 & 18,778 & 19,715 & 20,289 & 20,401 \\
\hline Natural gas & 620 & 653 & 799 & 646 & 667 & 698 & 701 & 777 & 779 & 835 & 909 & 957 \\
\hline Electricity & 2,460 & 2,400 & 2,400 & 2,410 & 2,410 & 2,410 & 2,410 & 2,410 & 2,400 & 2,410 & 2,410 & 2,410 \\
\hline Coal & 579 & 576 & 563 & 552 & 550 & 542 & 537 & 535 & 526 & 525 & 525 & 516 \\
\hline Gasoline & 30,643 & 26,900 & 24,451 & 24,429 & 26,305 & 26,322 & 26,403 & 32,214 & 31,427 & 31,790 & 33,790 & 34,505 \\
\hline Diesel & 19,716 & 18,473 & 18,683 & 18,621 & 19,590 & 19,601 & 20,344 & 24,671 & 26,846 & 28,276 & 30,057 & 31,767 \\
\hline Mazuth & 7,978 & 7,843 & 7,914 & 7,913 & 7,067 & 7,071 & 7,075 & 9,399 & 9,790 & 10,841 & 10,597 & 11,109 \\
\hline Heating (Gcal) & 1,234 & 1,235 & 1,234 & 1,236 & 1,236 & 1,236 & 1,238 & 1,238 & 1,238 & 1,272 & 1,271 & 1,271 \\
\hline Liquid petroleum gas (mt) & 7,199 & 7,769 & 7,404 & 7,294 & 7,224 & 7,230 & 7,236 & 9,075 & 10,919 & 10,918 & 12,163 & 12,515 \\
\hline \multicolumn{13}{|l|}{2001} \\
\hline Crude oil & 15,179 & 16,150 & 16,727 & 15,171 & 15,603 & 16,221 & 15,795 & 15,229 & 15,501 & 15,206 & 14,546 & 13,567 \\
\hline Natural gas & 1,111 & 1,157 & 1,200 & 1,214 & 1,208 & 1,207 & 1,207 & 1,213 & 1,212 & 1,209 & 1,331 & 1,265 \\
\hline Electricity & 2,630 & 2,630 & 2,630 & 2,640 & 2,640 & 2,640 & 2,640 & 2,640 & 2,640 & 2,642 & 2,654 & 2,660 \\
\hline Coal & 425 & 440 & 460 & 458 & 475 & 477 & 456 & 464 & 496 & 544 & 562 & 594 \\
\hline Gasoline & 35,400 & 33,782 & 31,419 & 27,755 & 29,515 & 30,508 & 33,742 & 33,837 & 34,915 & 34,979 & 35,127 & 27,937 \\
\hline Diesel & 32,057 & 31,080 & 30,313 & 29,124 & 24,946 & 23,363 & 26,276 & 26,333 & 26,494 & 28,487 & 27,088 & 21,402 \\
\hline Mazuth & 9,890 & 8,634 & 7,674 & 7,671 & 7,146 & 7,186 & 8,754 & 8,776 & 8,882 & 9,838 & 9,867 & 5,450 \\
\hline Heating (Gcal) & 1,423 & 1,423 & 1,423 & 1,421 & 1,421 & 1,421 & 1,427 & 1,427 & 1,427 & 1,427 & 1,427 & 1,427 \\
\hline Liquid petroleum gas (mt) & 13,301 & 13,319 & 12,121 & 12,129 & 12,175 & 13,425 & 15,176 & 15,222 & 15,279 & 15,310 & 15,367 & 14,215 \\
\hline \multicolumn{13}{|l|}{2002} \\
\hline Crude oil & 11,910 & 12,012 & 12,446 & 13,763 & 14,642 & 14,510 & 15,145 & 15,761 & 16,606 & 17,554 & 16,818 & 15,791 \\
\hline Natural gas & 1,288 & 1,440 & 1,441 & 1,443 & 1,444 & 1,445 & 1,447 & 1,449 & 1,358 & 1,356 & 1,336 & 1,341 \\
\hline Electricity & 2,774 & 2,787 & 2,802 & 2,831 & 2,861 & 2,861 & 2,897 & 2,900 & 2,900 & 2,900 & 2,903 & 2,903 \\
\hline Coal & 603 & 599 & 592 & 590 & 592 & 592 & 591 & 590 & 586 & 588 & 584 & 579 \\
\hline Gasoline & 26,720 & 23,437 & 22,294 & 22,200 & 22,062 & 22,209 & 22,050 & 22,991 & 23,361 & 23,589 & 23,523 & 23,147 \\
\hline Diesel & 19,786 & 18,362 & 18,141 & 18,044 & 17,853 & 18,534 & 18,534 & 18,463 & 18,557 & 18,186 & 18,128 & 18,012 \\
\hline Mazuth & 5,447 & 5,499 & 5,417 & 5,270 & 5,355 & 5,312 & 5,312 & 5,875 & 5,875 & 6,054 & 6,054 & 6,054 \\
\hline Heating (Gcal) & 1,354 & 1,352 & 1,359 & 1,361 & 1,360 & 1,361 & 1,361 & 1,361 & 1,361 & 1,376 & 1,376 & 1,376 \\
\hline Liquid petroleum gas (mt) & 13,038 & 13,182 & 13,182 & 13,182 & 13,184 & 13,039 & 13,039 & 13,196 & 13,196 & 13,196 & 13,196 & 13,196 \\
\hline \multicolumn{13}{|l|}{2003} \\
\hline Crude oil & 17,232 & 17,880 & 17,821 & 16,236 & 15,287 & 14,319 & 14,866 & 15,258 & 15,715 & 15,452 & 15,766 & 15,605 \\
\hline Natural gas & 1,504 & 1,581 & 1,569 & 1,576 & 1,573 & 1,558 & 1,546 & 1,547 & 1,580 & 1,581 & 1,564 & 1,568 \\
\hline Electricity & 2,902 & 2,905 & 2,905 & 2,915 & 2,921 & 2,918 & 2,895 & 2,897 & 2,897 & 3,002 & 3,014 & 2,997 \\
\hline Coal & 578 & 574 & 561 & 570 & 571 & 562 & 563 & 562 & 570 & 577 & 576 & 592 \\
\hline Gasoline & 23,311 & 23,440 & 23,254 & 22,938 & 22,696 & 22,667 & 22,466 & 22,418 & 22,419 & 22,256 & 23,039 & 23,037 \\
\hline Diesel & 18,319 & 18,841 & 18,770 & 18,553 & 18,553 & 18,420 & 18,286 & 18,547 & 18,599 & 18,316 & 18,245 & 18,245 \\
\hline Mazuth & 5,989 & 5,989 & 5,989 & 5,989 & 5,989 & 5,989 & 5,989 & 5,989 & 5,989 & 5,989 & 5,989 & 5,989 \\
\hline Heating (Gcal) & 1,369 & 1,564 & 1,559 & 1,654 & 1,403 & 1,404 & 1,355 & 1,357 & 1,279 & 1,369 & 1,359 & 1,359 \\
\hline Liquid petroleum gas $(\mathrm{mt})$ & 13,200 & 13,200 & 13,200 & 13,200 & 13,200 & 13,200 & 13,200 & 13,200 & 13,200 & 13,200 & 13,200 & 13,200 \\
\hline
\end{tabular}

Source: National Statistical Agency.

1/ Producers' ex-factory prices. Average prices for all customers. 
Table 6. Kazakhstan: Employment, 1999-2003 1/

\begin{tabular}{|c|c|c|c|c|c|}
\hline & 1999 & 2000 & 2001 & $\begin{array}{r}2002 \\
P\end{array}$ & $\begin{array}{l}2003 \\
\text { iminary }\end{array}$ \\
\hline & \multicolumn{5}{|c|}{ (Thousands) } \\
\hline Total & 6,105 & 6,201 & 6,699 & 6,709 & 6,968 \\
\hline Agriculture and forestry & 1,335 & 1,941 & 2,366 & 2,367 & 2,494 \\
\hline Fishing & 7 & 8 & 13 & 14 & 17 \\
\hline $\begin{array}{l}\text { Total industry } \\
\text { of which: }\end{array}$ & \multicolumn{4}{|c|}{ of which: } & 847 \\
\hline Mining & 129 & 137 & 167 & 167 & 187 \\
\hline Manufacturing & 628 & 573 & 514 & 504 & 492 \\
\hline Electricity, gas, and water: production and distribution & 148 & 146 & 150 & 153 & 169 \\
\hline Construction & 211 & 226 & 264 & 268 & 339 \\
\hline Trade, repair of cars and household goods & 1,398 & 971 & 1,006 & 1,007 & 990 \\
\hline Hotels and restaurants & 70 & 61 & 54 & 57 & 71 \\
\hline Transport and communication & 576 & 550 & 506 & 504 & 506 \\
\hline Financial sector & 36 & 40 & 46 & 50 & 55 \\
\hline Real estate & 211 & 226 & 214 & 203 & 164 \\
\hline Public administration & 344 & 314 & 281 & 280 & 325 \\
\hline Education & 513 & 531 & 576 & 589 & 629 \\
\hline Health and social services & 320 & 292 & 287 & 293 & 296 \\
\hline Community, social, and personal service activities & 172 & 181 & 183 & 186 & 193 \\
\hline \multirow[t]{2}{*}{ Services to households } & 8 & 6 & 71 & 67 & 43 \\
\hline & \multicolumn{5}{|c|}{ (In percent of total) } \\
\hline Total & 100.0 & 100.0 & 100.0 & 100.0 & 100.0 \\
\hline Agriculture and forestry & 21.9 & 31.3 & 35.3 & 35.3 & 35.8 \\
\hline Fishing & 0.1 & 0.1 & 0.2 & 0.2 & 0.2 \\
\hline Total industry & 14.8 & 13.8 & 12.4 & 12.3 & 12.2 \\
\hline \multicolumn{6}{|l|}{ of which: } \\
\hline Mining & 2.1 & 2.2 & 2.5 & 2.5 & 2.7 \\
\hline Manufacturing & 10.3 & 9.2 & 7.7 & 7.5 & 7.0 \\
\hline Electricity, gas, and water: production and distribution & 2.4 & 2.4 & 2.2 & 2.3 & 2.4 \\
\hline Construction & 3.4 & 3.6 & 3.9 & 4.0 & 4.9 \\
\hline Trade, repair of cars and household goods & 22.9 & 15.7 & 15.0 & 15.0 & 14.2 \\
\hline Hotels and restaurants & 1.1 & 1.0 & 0.8 & 0.8 & 1.0 \\
\hline Transport and communication & 9.4 & 8.9 & 7.6 & 7.5 & 7.3 \\
\hline Financial sector & 0.6 & 0.6 & 0.7 & 0.7 & 0.8 \\
\hline Real estate & 3.5 & 3.6 & 3.2 & 3.0 & 2.4 \\
\hline Public administration & 5.6 & 5.1 & 4.2 & 4.2 & 4.7 \\
\hline Education & 8.4 & 8.6 & 8.6 & 8.8 & 9.0 \\
\hline Health and social services & 5.2 & 4.7 & 4.3 & 4.4 & 4.2 \\
\hline Community, social, and personal service activities & 2.8 & 2.9 & 2.7 & 2.8 & 2.8 \\
\hline Services to households & 0.1 & 0.1 & 1.1 & 1.0 & 0.6 \\
\hline
\end{tabular}

Source: National Statistical Agency.

1/ Includes self-employment. 


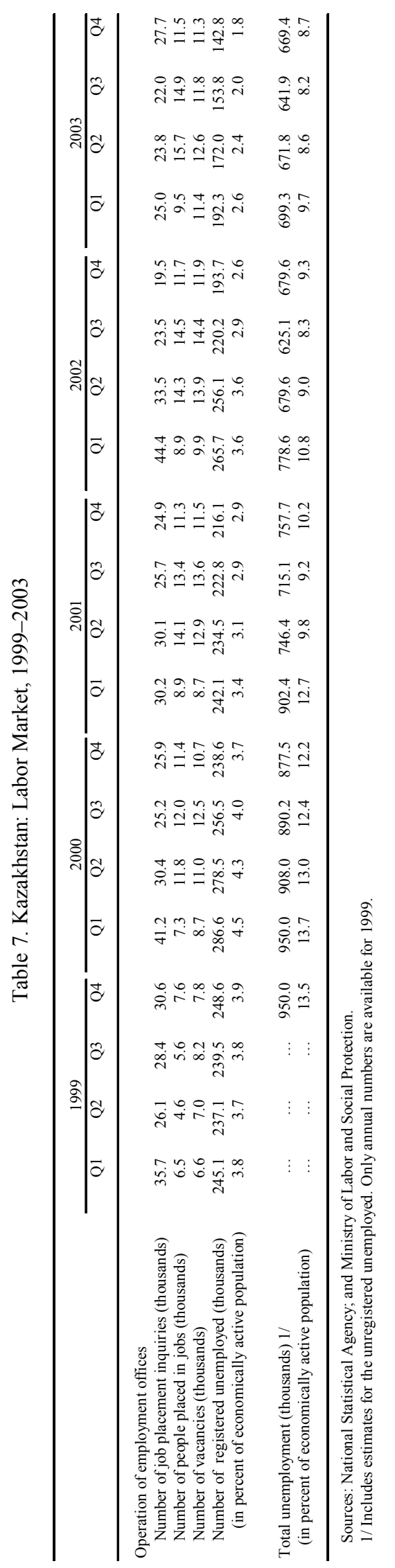




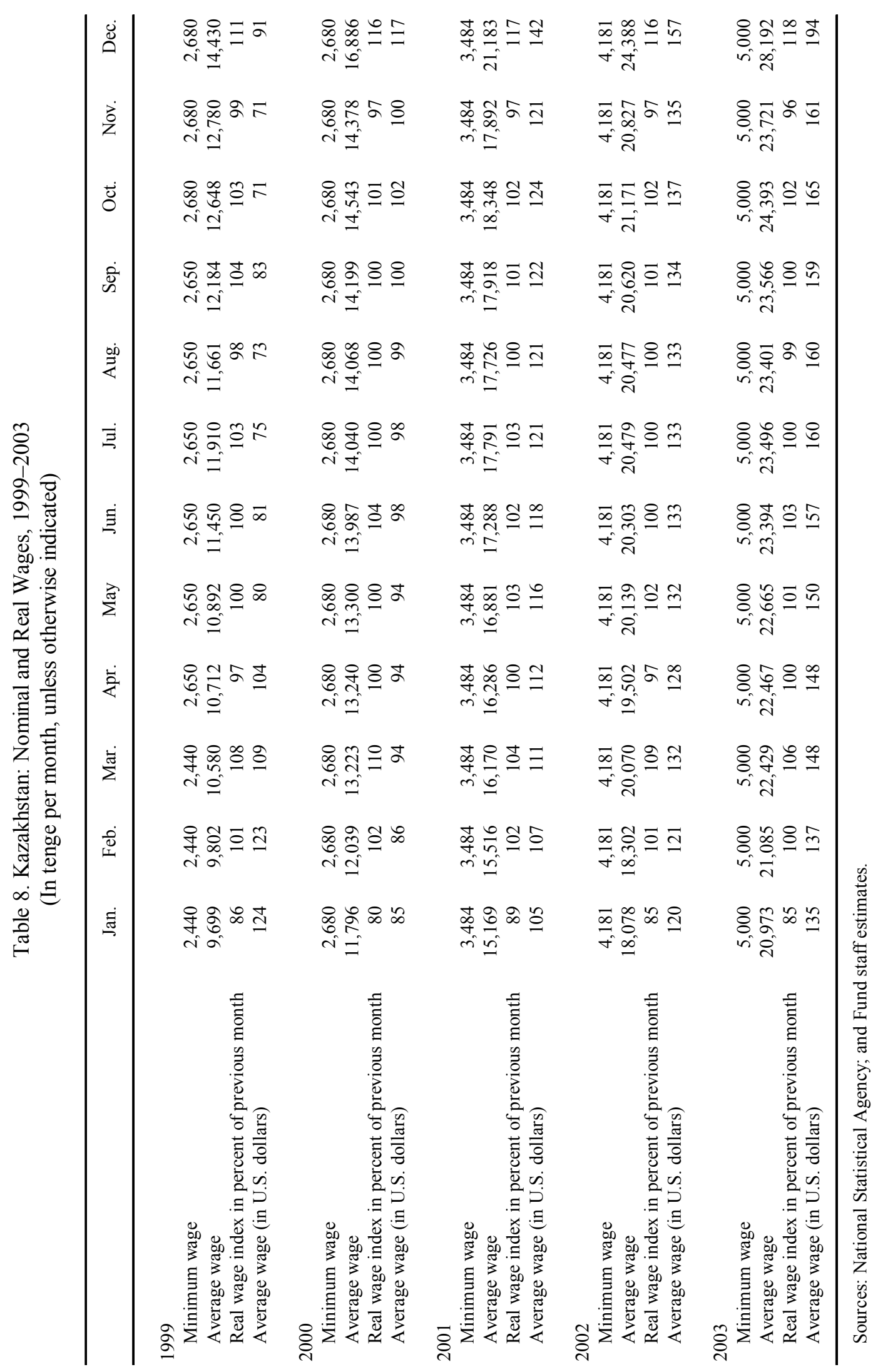


Table 9. Kazakhstan: Monthly Wages by Sector, 1999-2003 1/ (In tenge)

\begin{tabular}{|c|c|c|c|c|c|}
\hline & 1999 & 2000 & 2001 & 2002 & 2003 \\
\hline Total average & 11,864 & 14,374 & 17,303 & 20,323 & 23,221 \\
\hline Agriculture and forestry & 4,600 & 5,657 & 6,851 & 8,163 & 9,650 \\
\hline Fishing & 5,917 & 6,812 & 7,562 & 8,685 & 9,847 \\
\hline Total industry & 16,370 & 20,647 & 23,812 & 25,280 & 29,699 \\
\hline $\begin{array}{l}\text { of which: } \\
\text { Mining }\end{array}$ & \multicolumn{4}{|c|}{ of which: } & 45707 \\
\hline Manufacturing & 13,821 & 17,717 & $\begin{array}{l}50,025 \\
19,982\end{array}$ & 22,130 & 24,918 \\
\hline Electricity, gas and water: production and distribution & 15,651 & 17,290 & 20,026 & 21,594 & 23,550 \\
\hline Construction & 15,905 & 21,017 & 26,805 & 32,453 & 33,951 \\
\hline Trade, car repair, and household goods & 10,766 & 12,961 & 15,366 & 18,931 & 23,077 \\
\hline Hotels and restaurants & 13,736 & 15,979 & 21,511 & 32,743 & 39,840 \\
\hline Transport and communication & 14,696 & 18,788 & 24,412 & 28,969 & 34,421 \\
\hline Financial sector & 33,392 & 36,140 & 41,686 & 50,460 & 56,592 \\
\hline Real estate & 12,338 & 16,672 & 22,132 & 29,329 & 36,851 \\
\hline Public administration & 11,308 & 11,758 & 14,970 & 16,930 & 18,066 \\
\hline Education & 8,149 & 8,512 & 9,937 & 12,863 & 14,432 \\
\hline Health and social services & 6,821 & 7,267 & 8,288 & 10,863 & 12,198 \\
\hline Other municipal, social, and personal services & 10,097 & 12,857 & 16,873 & 21,078 & 24,383 \\
\hline
\end{tabular}

Source: National Statistical Agency.

1/ Data are not comparable with monthly wages in Table 8, since only the annual data are revised when new information becomes available. 


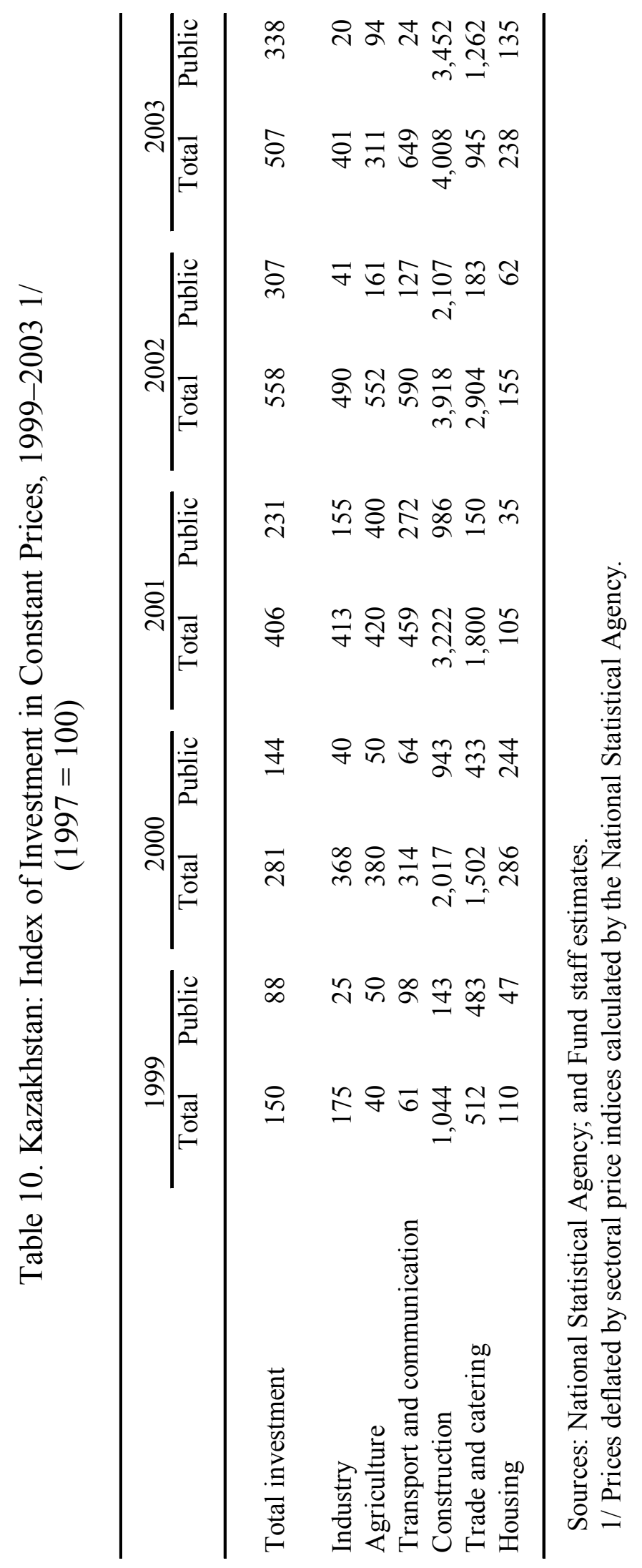




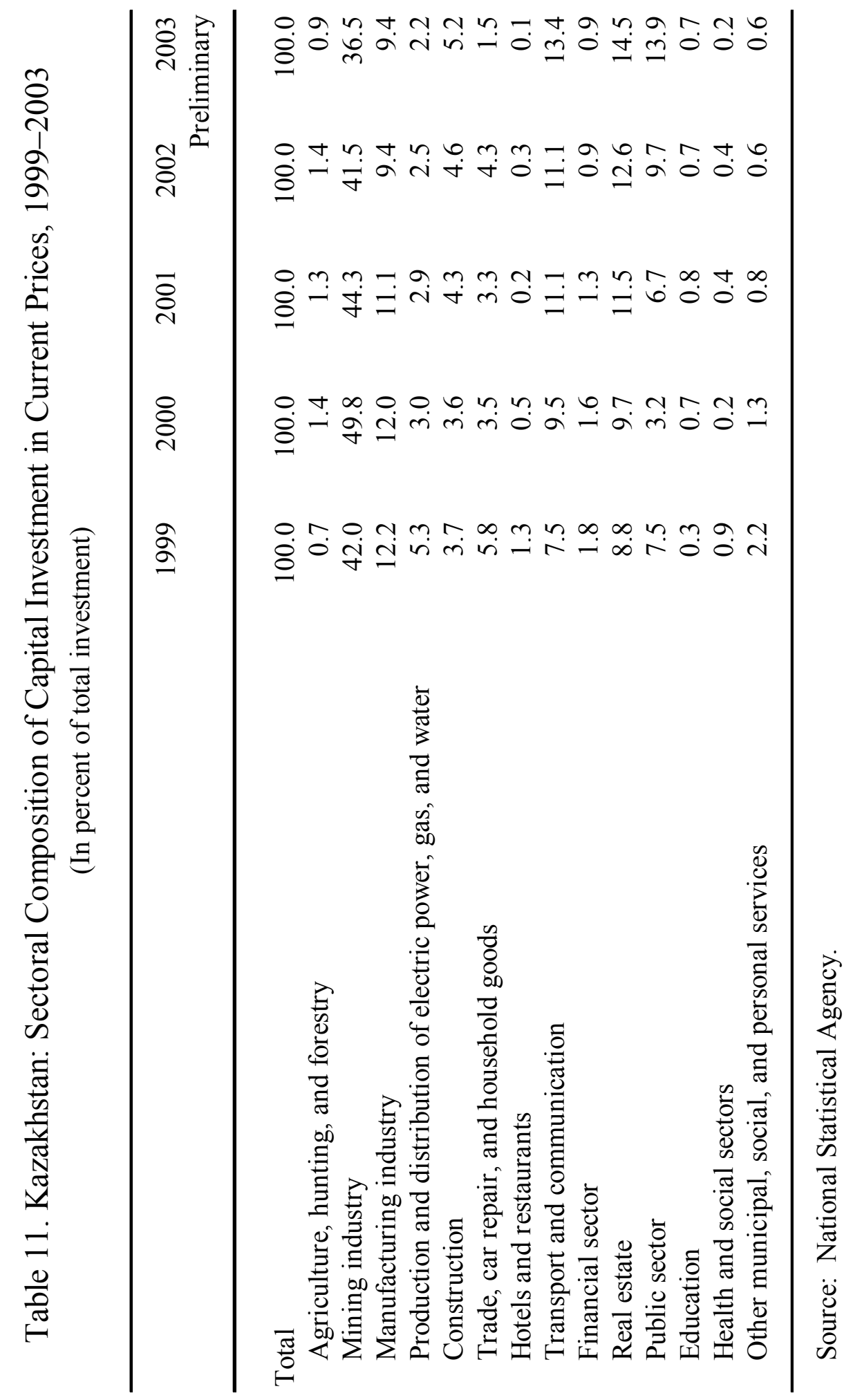


Table 12. Kazakhstan: Savings Investment Balance, 1999-2003 1/

\begin{tabular}{|c|c|c|c|c|c|}
\hline & 1999 & 2000 & 2001 & \multicolumn{2}{|c|}{ Preliminary } \\
\hline & \multicolumn{5}{|c|}{ (In percent of GDP by final use) } \\
\hline Consumption & 80.7 & 73.3 & 73.3 & 71.3 & 67.4 \\
\hline Net exports & 2.3 & 8.6 & -0.9 & 0.9 & 6.2 \\
\hline Investment & 17.1 & 18.1 & 27.6 & 27.8 & 26.4 \\
\hline Public & 1.7 & 2.0 & 3.1 & 3.4 & 4.6 \\
\hline Private & 13.8 & 15.3 & 21.3 & 21.1 & 19.0 \\
\hline Change in inventories & 1.5 & 0.8 & 3.3 & 3.3 & 2.7 \\
\hline Savings & 17.1 & 18.1 & 27.6 & 27.8 & 26.4 \\
\hline Domestic savings & 16.9 & 21.3 & 23.6 & 24.3 & 26.2 \\
\hline Public & -2.7 & 1.5 & 6.5 & 5.4 & 9.0 \\
\hline Private & 19.5 & 19.8 & 17.0 & 19.0 & 17.1 \\
\hline Foreign savings & 0.1 & -3.2 & 4.0 & 3.5 & 0.2 \\
\hline
\end{tabular}

Sources: Kazakhstani authorities; and Fund staff estimates.

1/ Data for 2000-02 include adjustments to reflect revisions of the balance of payments by the National Bank and the staff. 2003 values are staff estimates based on preliminary data for GDP by production, balance of payments, and investment from enterprise surveys. 


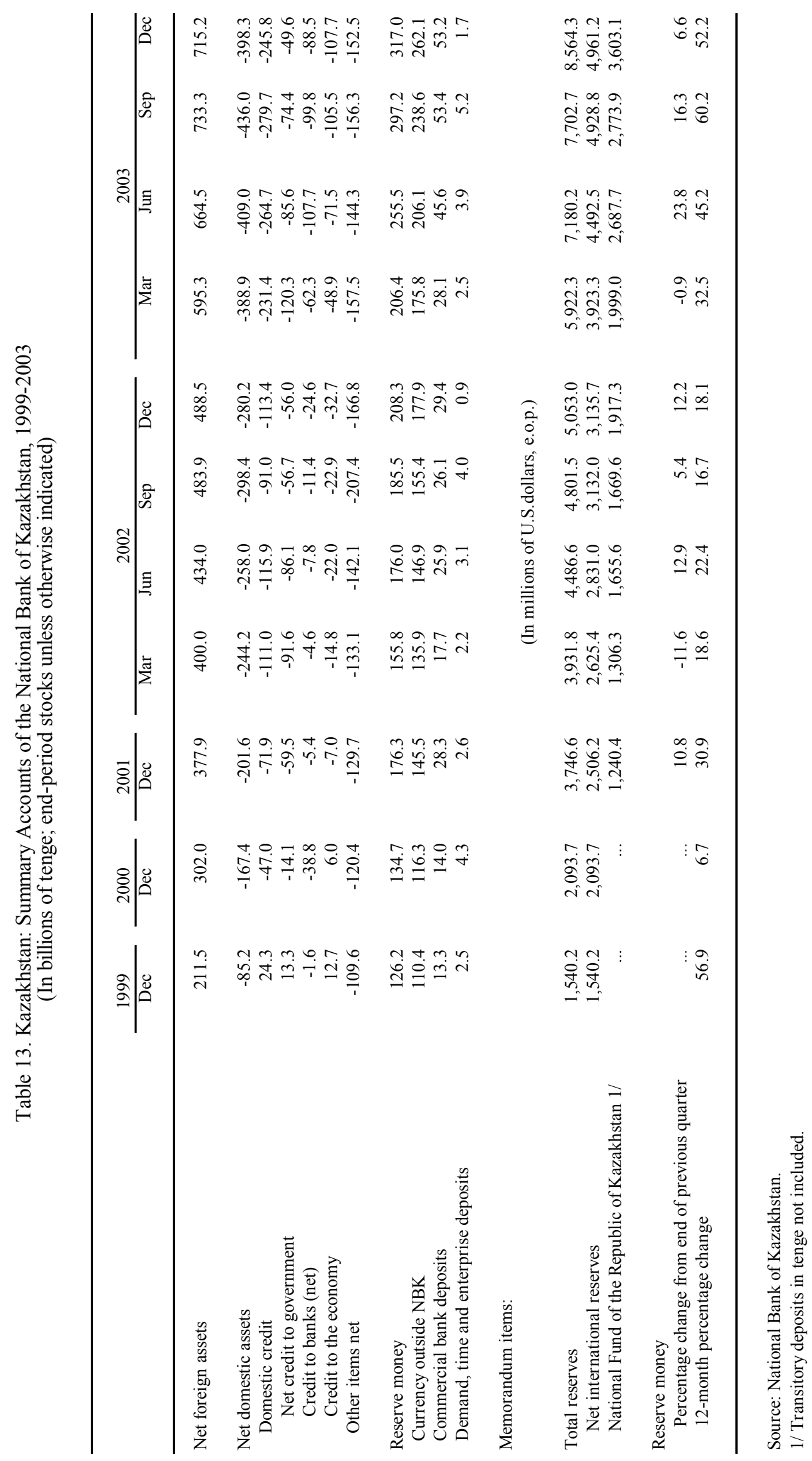




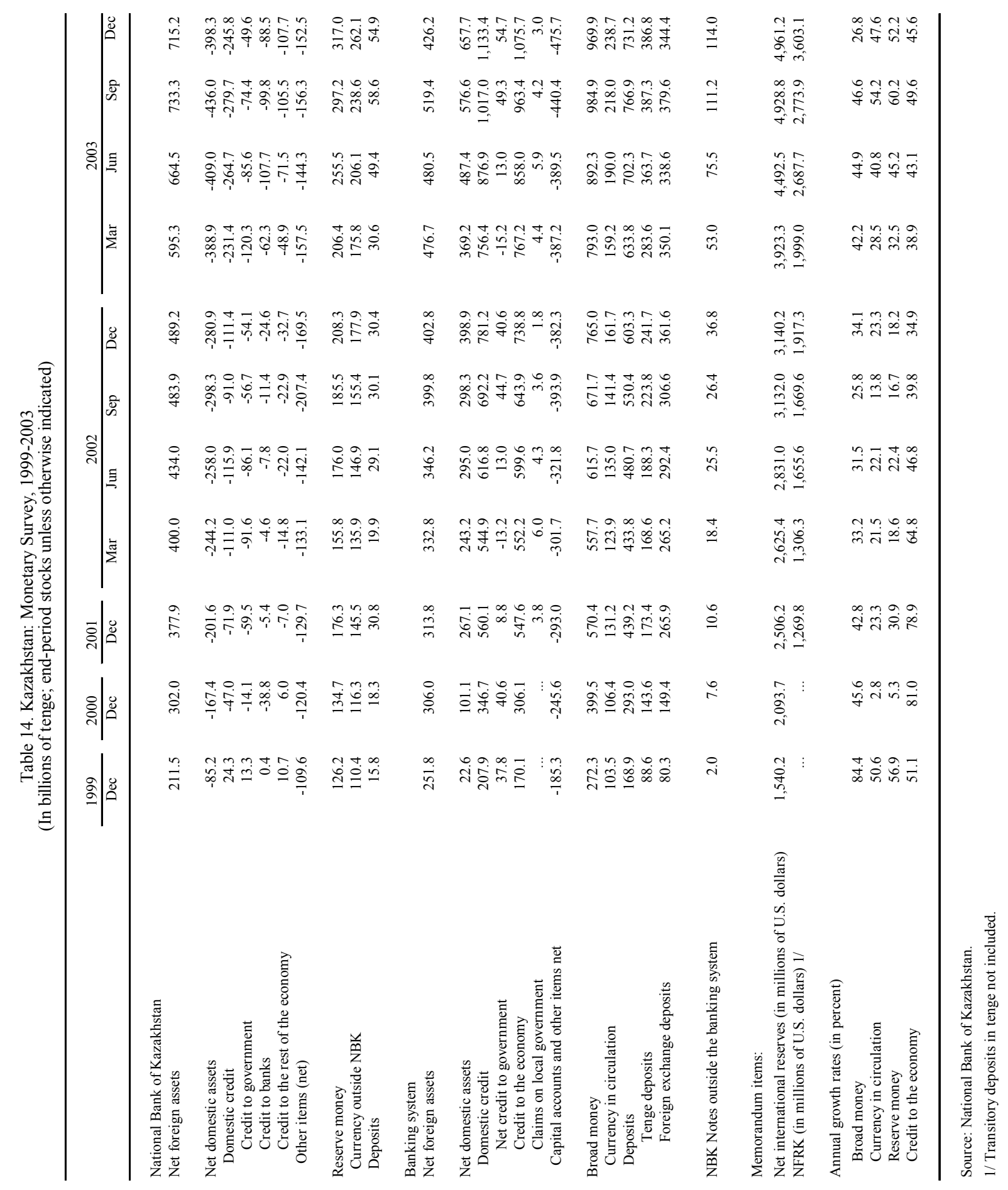


Table 15. Kazakhstan: Interest Rates, 1999-2003

(In percent; end-of-period)

\begin{tabular}{|c|c|c|c|c|c|c|c|}
\hline & & \multirow{2}{*}{$\begin{array}{c}\text { Inflation } \\
\text { 12-Month Average }\end{array}$} & \multirow{2}{*}{$\begin{array}{c}\text { NBK Refinance } \\
\text { Rate }\end{array}$} & \multirow{2}{*}{$\begin{array}{l}\text { Yield on 3-Month } \\
\text { Treasury Bills 1/ }\end{array}$} & \multirow{2}{*}{$\begin{array}{l}\text { Commercial Bank Short- } \\
\text { Term Lending Rates 2/ }\end{array}$} & \multicolumn{2}{|c|}{ Commercial Bank Time Deposit Rates 2} \\
\hline & & & & & & Households & Legal Entities \\
\hline \multirow[t]{12}{*}{1999} & January & 1.0 & 25.0 & 26.3 & 18.3 & 17.2 & 10.7 \\
\hline & February & -0.3 & 25.0 & 26.3 & 19.8 & 17.4 & 13.8 \\
\hline & March & -1.2 & 25.0 & 26.3 & 22.5 & 18.8 & 15.2 \\
\hline & April & 2.8 & 25.0 & $\ldots$ & 24.7 & 13.3 & 12.0 \\
\hline & May & 3.9 & 25.0 & $\ldots$ & 24.2 & 13.1 & 9.1 \\
\hline & June & 9.8 & 25.0 & $\ldots$ & 25.1 & 14.1 & 9.7 \\
\hline & July & 11.2 & 22.0 & 21.6 & 25.6 & 16.2 & 8.1 \\
\hline & August & 11.9 & 20.0 & 21.6 & 24.9 & 16.5 & 5.6 \\
\hline & September & 12.8 & 20.0 & $\ldots$ & 26.7 & 25.6 & 8.7 \\
\hline & October & 14.3 & 20.0 & $\ldots$ & 28.1 & 18.7 & 9.4 \\
\hline & November & 16.3 & 18.0 & 16.6 & 23.3 & 20.2 & 7.9 \\
\hline & December & 17.8 & 18.0 & 16.6 & 21.4 & 13.4 & 7.9 \\
\hline \multirow[t]{12}{*}{2000} & January & 19.8 & 18.0 & 16.7 & 19.7 & 16.5 & 9.4 \\
\hline & February & 20.2 & 18.0 & 16.4 & 21.9 & 16.7 & 10.1 \\
\hline & March & 20.4 & 16.0 & 16.0 & 22.3 & 10.5 & 6.7 \\
\hline & April & 15.6 & 16.0 & 15.6 & 22.0 & 17.6 & 6.4 \\
\hline & May & 14.7 & 16.0 & 14.6 & 20.7 & 20.0 & 7.5 \\
\hline & June & 10.2 & 14.0 & 13.1 & 20.3 & 16.5 & 7.8 \\
\hline & July & 9.5 & 14.0 & 12.6 & 20.2 & 18.6 & 4.1 \\
\hline & August & 10.0 & 14.0 & 9.9 & 19.4 & 18.0 & 4.4 \\
\hline & September & 9.8 & 14.0 & 9.5 & 20.2 & 16.2 & 5.0 \\
\hline & October & 10.4 & 14.0 & 7.6 & 20.6 & 15.5 & 6.2 \\
\hline & November & 10.2 & 14.0 & 7.5 & 18.2 & 15.9 & 6.4 \\
\hline & December & 9.8 & 14.0 & 6.8 & 19.9 & 15.6 & 6.1 \\
\hline \multirow[t]{12}{*}{2001} & January & 8.2 & 14.0 & 6.7 & 18.8 & 16.4 & 3.7 \\
\hline & February & 8.8 & 12.5 & 6.6 & 18.6 & 14.7 & 7.6 \\
\hline & March & 9.6 & 12.5 & 5.6 & 20.2 & 15.7 & 7.7 \\
\hline & April & 9.2 & 12.5 & 5.4 & 19.8 & 14.6 & 6.5 \\
\hline & May & 9.3 & 12.5 & 5.2 & 20.2 & 14.7 & 6.2 \\
\hline & June & 9.2 & 12.0 & 5.0 & 18.1 & 13.9 & 6.4 \\
\hline & July & 9.1 & 12.0 & 4.9 & 17.0 & 14.4 & 4.5 \\
\hline & August & 9.0 & 12.0 & 4.8 & 18.2 & 14.4 & 5.8 \\
\hline & September & 8.9 & 11.0 & 5.1 & 18.8 & 13.7 & 5.7 \\
\hline & October & 8.7 & 11.0 & 5.4 & 18.1 & 13.8 & 5.1 \\
\hline & November & 8.5 & 9.0 & $\ldots$ & 17.1 & 13.6 & 5.5 \\
\hline & December & 8.4 & 9.0 & $\ldots$ & 16.4 & 12.8 & 5.6 \\
\hline \multirow[t]{12}{*}{2002} & January & 6.0 & 9.0 & 5.3 & 14.5 & 13.1 & 5.3 \\
\hline & February & 5.8 & 9.0 & $\ldots$ & 16.1 & 12.5 & 5.2 \\
\hline & March & 5.6 & 8.0 & $\ldots$ & 16.2 & 11.2 & 5.1 \\
\hline & April & 5.4 & 8.0 & 5.3 & 15.7 & 11.5 & 5.0 \\
\hline & May & 5.4 & 8.0 & 5.3 & 16.1 & 11.3 & 4.8 \\
\hline & June & 5.5 & 8.0 & $\ldots$ & 15.3 & 11.1 & 4.7 \\
\hline & July & 5.6 & 8.0 & $\ldots$ & 15.6 & 11.3 & 5.1 \\
\hline & August & 5.7 & 8.0 & $\ldots$ & 21.3 & 10.2 & 4.9 \\
\hline & September & 5.7 & 8.0 & $\ldots$ & 15.7 & 9.4 & 5.5 \\
\hline & October & 5.8 & 8.0 & $\ldots$ & 15.6 & 10.8 & 5.3 \\
\hline & November & 5.8 & 7.5 & $\ldots$ & 17.1 & 12.0 & 5.3 \\
\hline & December & 5.9 & 7.5 & $\ldots$ & 14.5 & 11.0 & 5.4 \\
\hline \multirow[t]{12}{*}{2003} & January & 6.9 & 7.5 & $\ldots$ & 14.6 & 11.2 & 5.2 \\
\hline & February & 7.0 & 7.5 & $\ldots$ & 16.0 & 11.3 & 5.3 \\
\hline & March & 7.0 & 7.5 & $\ldots$ & 15.9 & 10.9 & 5.0 \\
\hline & April & 7.0 & 7.5 & $\ldots$ & 17.6 & 9.5 & 5.4 \\
\hline & May & 6.9 & 7.5 & $\ldots$ & 16.6 & 11.2 & 4.4 \\
\hline & June & 6.7 & 7.5 & $\ldots$ & 15.7 & 11.1 & 3.5 \\
\hline & July & 6.4 & 7.0 & $\ldots$ & 17.2 & 10.9 & 3.8 \\
\hline & August & 6.3 & 7.0 & $\ldots$ & 16.6 & 9.9 & 3.8 \\
\hline & September & 6.3 & 7.0 & 6.0 & 16.9 & 10.5 & 3.7 \\
\hline & October & 6.3 & 7.0 & $\ldots$ & 15.5 & 10.0 & 2.8 \\
\hline & November & 6.4 & 7.0 & $\ldots$ & 15.7 & 10.0 & 3.0 \\
\hline & December & 6.4 & 7.0 & $\ldots$ & 15.5 & 10.9 & 3.5 \\
\hline
\end{tabular}

Source: National Bank of Kazakhstan.

$1 /$ The absence of entry indicates that T-bills were not issued.

2/ Rates on new credits and deposits in tenge. 
Table 16. Kazakhstan: Stock Exchange (KASE) Weighted Average Exchange Rates, 1999-2003 1/ (End-of-period)

\begin{tabular}{|c|c|c|c|c|c|c|c|}
\hline & & \multicolumn{2}{|c|}{ Tenge per U.S. dollar } & \multicolumn{2}{|c|}{ Tenge per Euro 2/ } & \multicolumn{2}{|c|}{ Tenge per Russian ruble } \\
\hline & & Period Average & End-of-Period & Period Average & End-of-Period & Period Average & End-of-Period \\
\hline \multirow[t]{12}{*}{1999} & January & 84.57 & 85.12 & $\ldots$ & $\ldots$ & $\ldots$ & $\ldots$ \\
\hline & February & 85.71 & 86.45 & $\cdots$ & $\cdots$ & $\cdots$ & $\cdots$ \\
\hline & March & 87.42 & 88.10 & $\ldots$ & $\ldots$ & $\ldots$ & $\ldots$ \\
\hline & April & 113.80 & 114.80 & 62.16 & 62.10 & $\ldots$ & $\ldots$ \\
\hline & May & 119.14 & 129.03 & 67.44 & 69.31 & $\ldots$ & $\ldots$ \\
\hline & June & 131.88 & 132.31 & 70.08 & 70.30 & $\cdots$ & $\cdots$ \\
\hline & July & 132.45 & 131.91 & 69.96 & 72.40 & $\ldots$ & $\ldots$ \\
\hline & August & 131.81 & 132.26 & 71.61 & 71.08 & $\ldots$ & $\cdots$ \\
\hline & September & 135.78 & 140.11 & 73.16 & 76.40 & $\ldots$ & $\ldots$ \\
\hline & October & 141.21 & 140.22 & 77.43 & 75.65 & $\ldots$ & $\ldots$ \\
\hline & November & 139.16 & 137.90 & 73.64 & 71.41 & $\ldots$ & $\ldots$ \\
\hline & December & 138.19 & 138.25 & 71.77 & 71.50 & $\ldots$ & $\ldots$ \\
\hline \multirow[t]{12}{*}{2000} & January & 139.06 & 139.38 & 72.15 & 70.78 & $\ldots$ & $\ldots$ \\
\hline & February & 139.90 & 140.44 & 70.77 & 71.20 & $\ldots$ & $\ldots$ \\
\hline & March & 141.42 & 141.95 & 70.24 & 70.30 & $\ldots$ & $\ldots$ \\
\hline & April & 142.21 & 142.01 & 69.47 & 66.78 & $\ldots$ & $\ldots$ \\
\hline & May & 142.29 & 142.30 & 66.63 & 65.90 & $\ldots$ & $\ldots$ \\
\hline & June & 142.65 & 142.86 & 68.70 & 68.70 & $\ldots$ & $\ldots$ \\
\hline & July & 142.79 & 142.71 & 69.32 & 69.50 & $\ldots$ & $\ldots$ \\
\hline & August & 142.60 & 142.52 & 65.92 & 65.75 & $\ldots$ & $\ldots$ \\
\hline & September & 142.69 & 142.58 & 63.28 & 62.44 & $\ldots$ & $\ldots$ \\
\hline & October & 142.57 & 142.58 & 63.81 & 62.50 & $\ldots$ & $\ldots$ \\
\hline & November & 144.01 & 144.15 & $\ldots$ & $\ldots$ & $\ldots$ & $\ldots$ \\
\hline & December & 144.98 & 145.40 & 65.40 & 65.30 & $\ldots$ & $\cdots$ \\
\hline \multirow[t]{12}{*}{2001} & January & 145.38 & 145.11 & $\ldots$ & $\ldots$ & $\ldots$ & $\ldots$ \\
\hline & February & 145.33 & 145.28 & $\ldots$ & $\ldots$ & 5.07 & 5.07 \\
\hline & March & 145.48 & 145.42 & 68.25 & 68.25 & 5.08 & 5.06 \\
\hline & April & 145.54 & 145.77 & $\ldots$ & $\ldots$ & 5.04 & 5.05 \\
\hline & May & 146.13 & 146.47 & 65.86 & 65.86 & 5.05 & 5.03 \\
\hline & June & 146.59 & 146.80 & $\ldots$ & $\ldots$ & 5.04 & 5.04 \\
\hline & July & 146.76 & 147.07 & $\ldots$ & $\ldots$ & 5.02 & 5.02 \\
\hline & August & 147.17 & 147.30 & $\ldots$ & $\ldots$ & 5.03 & 5.03 \\
\hline & September & 147.70 & 147.80 & $\ldots$ & $\ldots$ & 5.01 & 5.02 \\
\hline & October & 148.03 & 148.13 & 132.85 & 132.85 & 5.01 & 5.01 \\
\hline & November & 148.56 & 148.95 & 130.80 & 130.80 & 5.00 & 4.97 \\
\hline & December & 150.32 & 150.94 & $\ldots$ & $\ldots$ & 5.00 & 5.00 \\
\hline \multirow[t]{12}{*}{2002} & January & 151.62 & 151.87 & $\ldots$ & $\ldots$ & 4.96 & 4.95 \\
\hline & February & 152.02 & 152.12 & $\ldots$ & $\ldots$ & 4.92 & 4.92 \\
\hline & March & 152.22 & 152.44 & 133.03 & 133.55 & 4.90 & 4.90 \\
\hline & April & 152.75 & 152.99 & $\ldots$ & $\ldots$ & 4.90 & 4.91 \\
\hline & May & 152.96 & 153.18 & $\ldots$ & $\ldots$ & 4.91 & 4.91 \\
\hline & June & 153.13 & 153.27 & $\ldots$ & $\ldots$ & 4.87 & 4.86 \\
\hline & July & 153.90 & 154.26 & $\ldots$ & $\ldots$ & 4.88 & 4.88 \\
\hline & August & 154.31 & 154.53 & $\ldots$ & $\ldots$ & 4.89 & 4.89 \\
\hline & September & 154.52 & 154.72 & $\ldots$ & $\ldots$ & 4.88 & 4.89 \\
\hline & October & 154.41 & 154.47 & $\ldots$ & $\ldots$ & 4.87 & 4.89 \\
\hline & November & 154.38 & 154.88 & $\ldots$ & $\ldots$ & 4.86 & 4.86 \\
\hline & December & 155.68 & 155.85 & $\ldots$ & $\ldots$ & 4.89 & 4.90 \\
\hline \multirow[t]{12}{*}{2003} & January & 155.34 & 154.83 & $\ldots$ & $\ldots$ & $\ldots$ & $\ldots$ \\
\hline & February & 153.34 & 151.66 & 166.31 & 164.50 & 4.85 & 4.80 \\
\hline & March & 151.52 & 152.10 & 161.55 & 161.55 & 4.81 & 4.81 \\
\hline & April & 151.98 & 151.76 & 163.70 & 163.70 & $\ldots$ & $\ldots$ \\
\hline & May & 150.98 & 150.41 & $\ldots$ & $\ldots$ & 4.90 & 4.90 \\
\hline & June & 149.01 & 147.68 & 170.80 & 170.80 & 4.90 & 4.88 \\
\hline & July & 146.96 & 146.76 & $\ldots$ & $\ldots$ & $\ldots$ & $\ldots$ \\
\hline & August & 146.72 & 147.47 & $\ldots$ & $\ldots$ & $\ldots$ & $\ldots$ \\
\hline & September & 148.00 & 148.97 & 166.51 & 166.63 & $\ldots$ & $\ldots$ \\
\hline & October & 147.82 & 147.77 & 174.51 & 175.22 & $\ldots$ & $\ldots$ \\
\hline & November & 146.99 & 146.63 & 174.86 & 174.86 & $\ldots$ & $\ldots$ \\
\hline & December & 144.70 & 143.33 & 178.49 & 178.84 & 4.90 & 4.90 \\
\hline
\end{tabular}

Source: National Bank of Kazakhstan.

1/ The absence of entry indicates that trade did not take place.

2/ Tenge per Deutsche Mark up to May 2001. 
Table 17. Kazakhstan: Number of Commercial Banks and Branches, 1999-2003 (End-of-period)

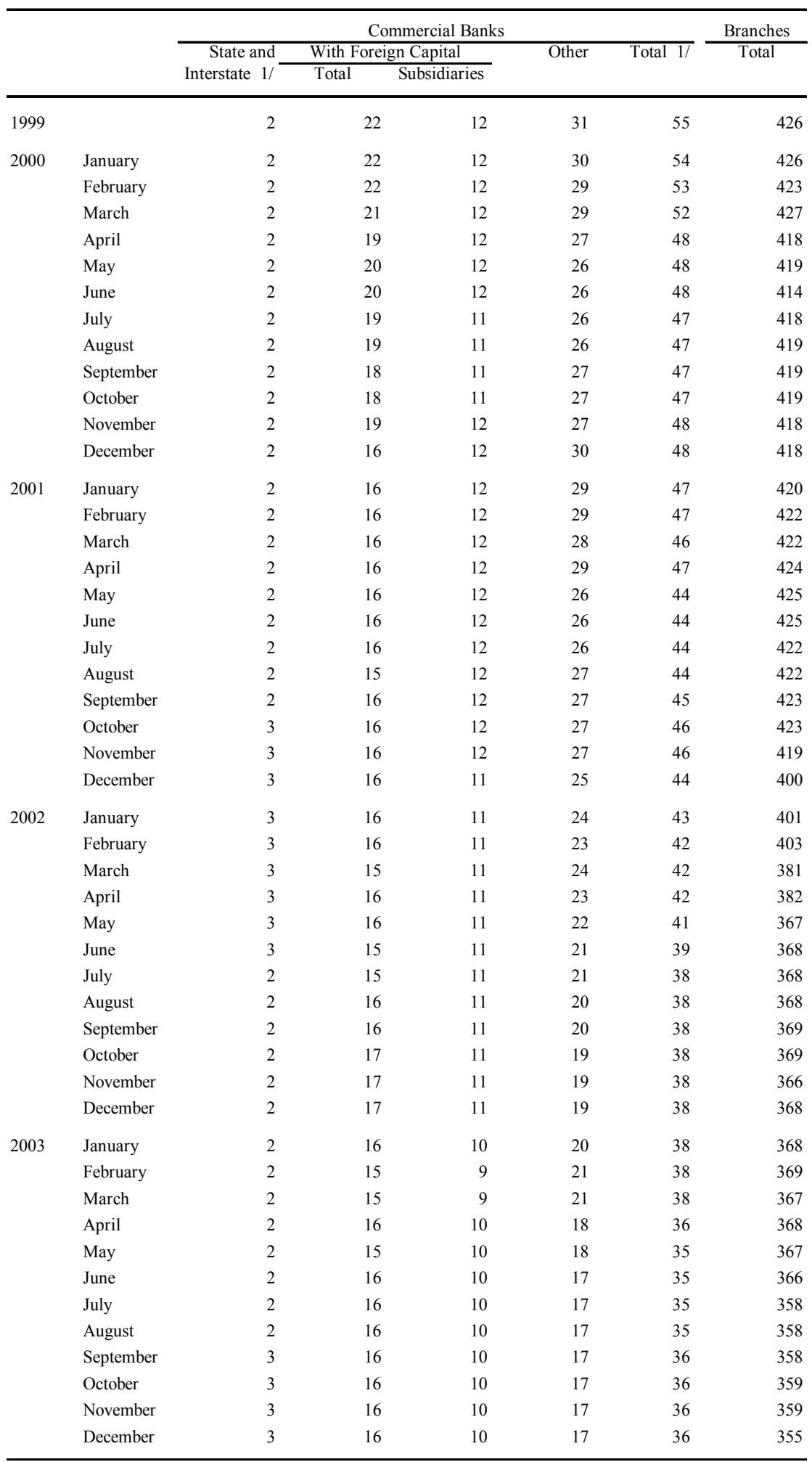

Source: National Bank of Kazakhstan.

1/ Including Development Bank, which does not take deposits from the public, and Eximbank which was privatized in February 2004. 
Table 18a. Kazakhstan: General Government Fiscal Operations, 1999-2003 (In billions of tenge)

\begin{tabular}{|c|c|c|c|c|c|c|c|c|c|c|c|}
\hline & \multirow[t]{2}{*}{1999} & \multirow[t]{2}{*}{2000} & \multirow[t]{2}{*}{2001} & \multicolumn{4}{|c|}{$20021 /$} & \multicolumn{4}{|c|}{$20031 /$} \\
\hline & & & & Q1 & Q2 & Q3 & $\overline{\mathrm{Q} 4}$ & Q1 & Q2 & Q3 & $\overline{\mathrm{Q} 4}$ \\
\hline Total revenues and grants & 354 & 565 & 834 & 177 & 381 & 607 & 848 & 270 & 547 & 823 & 1,169 \\
\hline Total revenues & 351 & 562 & 833 & 177 & 381 & 607 & 848 & 270 & 547 & 823 & 1,169 \\
\hline Oil revenues & 19 & 86 & 215 & 31 & 52 & 101 & 165 & 70 & 111 & 161 & 275 \\
\hline Non-oil revenues & 332 & 476 & 618 & 146 & 330 & 506 & 682 & 201 & 435 & 662 & 894 \\
\hline Current revenues & 350 & 560 & 825 & 176 & 380 & 601 & 838 & 269 & 544 & 815 & 1,157 \\
\hline Capital revenues & 1 & 2 & 9 & 1 & 2 & 5 & 9 & 1 & 3 & 9 & 13 \\
\hline Tax revenues & 323 & 521 & 724 & 168 & 351 & 562 & 793 & 260 & 483 & 741 & 1,071 \\
\hline Income tax & 90 & 215 & 305 & 69 & 134 & 218 & 322 & 127 & 202 & 312 & 445 \\
\hline Corporate income tax & 55 & 164 & 237 & 53 & 98 & 162 & 244 & 107 & 159 & 248 & 352 \\
\hline Oil revenues & 7 & 67 & 111 & 20 & 27 & 62 & 97 & 47 & 60 & 89 & 157 \\
\hline Non-oil revenues & 47 & 96 & 126 & 33 & 70 & 100 & 147 & 60 & 99 & 159 & 195 \\
\hline Personal income tax & 35 & 51 & 69 & 16 & 36 & 56 & 77 & 20 & 43 & 66 & 93 \\
\hline Social tax & 70 & 99 & 124 & 28 & 61 & 95 & 134 & 34 & 72 & 111 & 158 \\
\hline Property taxes & 25 & 27 & 33 & 8 & 18 & 26 & 35 & 9 & 19 & 29 & 38 \\
\hline Domestic taxes on goods, works and services & 125 & 162 & 234 & 54 & 119 & 192 & 258 & 80 & 166 & 252 & 380 \\
\hline VAT & 90 & 115 & 160 & 38 & 87 & 140 & 176 & 52 & 102 & 161 & 231 \\
\hline Excises & 19 & 19 & 22 & 6 & 12 & 19 & 25 & 5 & 13 & 20 & 27 \\
\hline Receipts from use of natural resources & 10 & 22 & 48 & 10 & 18 & 31 & 54 & 22 & 50 & 69 & 118 \\
\hline Oil revenues $2 /$ & 5 & 13 & 41 & 6 & 9 & 17 & 35 & 16 & 38 & 53 & 85 \\
\hline Non-oil revenues & 5 & 9 & 7 & 4 & 10 & 14 & 19 & 6 & 12 & 16 & 34 \\
\hline Business and professional licences & 7 & 5 & 5 & 1 & 1 & 2 & 3 & 1 & 2 & 3 & 3 \\
\hline Taxes on international trade and external transactions & 12 & 18 & 26 & 7 & 16 & 26 & 39 & 9 & 20 & 31 & 42 \\
\hline Other taxes & 1 & 1 & 1 & 2 & 3 & 5 & 5 & 2 & 4 & 6 & 8 \\
\hline Non-tax revenues $3 /$ & 27 & 39 & 100 & 8 & 29 & 39 & 46 & 9 & 61 & 74 & 86 \\
\hline Income from capital transactions & 1 & 2 & 9 & 1 & 2 & 5 & 9 & 1 & 3 & 9 & 13 \\
\hline Grants & 3 & 3 & 1 & 0 & 0 & 0 & 0 & 0 & 0 & 0 & 0 \\
\hline Total expenditures and net lending & 458 & 584 & 746 & 141 & 350 & 549 & 794 & 186 & 453 & 746 & 1,036 \\
\hline Total expenditures & 440 & 567 & 725 & 138 & 339 & 531 & 776 & 181 & 439 & 701 & 986 \\
\hline Current expenditures & 413 & 524 & 634 & 126 & 301 & 457 & 649 & 155 & 358 & 551 & 764 \\
\hline Expenditures on goods and services & $\ldots$ & $\ldots$ & 402 & $\ldots$ & 180 & 278 & 403 & 96 & 226 & 348 & 478 \\
\hline Wages & $\ldots$ & $\ldots$ & 106 & $\ldots$ & 65 & 95 & 134 & 34 & 76 & 111 & 150 \\
\hline Employers' contributions & $\ldots$ & $\ldots$ & 16 & $\ldots$ & 9 & 13 & 18 & 5 & 11 & 16 & 21 \\
\hline Goods and services & $\ldots$ & $\ldots$ & 254 & $\ldots$ & 93 & 148 & 218 & 49 & 119 & 188 & 261 \\
\hline Other expenditures on goods and services & $\ldots$ & $\ldots$ & 25 & $\ldots$ & 13 & 22 & 32 & 8 & 20 & 34 & 47 \\
\hline Interest & 19 & 35 & 38 & 5 & 19 & 24 & 39 & 4 & 18 & 22 & 35 \\
\hline Domestic & $\ldots$ & 9 & 9 & 1 & 4 & 6 & 10 & 2 & 6 & 7 & 12 \\
\hline Foreign & $\ldots$ & 26 & 29 & 4 & 15 & 18 & 29 & 1 & 12 & 14 & 23 \\
\hline Current transfers & $\ldots$ & $\ldots$ & 194 & $\ldots$ & 103 & 154 & 208 & 56 & 114 & 181 & 251 \\
\hline Capital expenditures & 27 & 43 & 91 & 13 & 38 & 74 & 126 & 26 & 81 & 150 & 222 \\
\hline Net lending & 18 & 17 & 21 & 3 & 11 & 17 & 18 & 5 & 14 & 45 & 51 \\
\hline Extending of credits; share participation & $\ldots$ & $\ldots$ & 34 & $\ldots$ & 16 & 23 & 32 & 7 & 20 & 54 & 68 \\
\hline Repayment of credits & $\ldots$ & $\ldots$ & 13 & $\ldots$ & 5 & 6 & 13 & 2 & 6 & 9 & 18 \\
\hline Overall budget balance & -101 & -20 & 88 & 36 & 31 & 58 & 54 & 84 & 93 & 78 & 133 \\
\hline Statistical discrepancy 4/ & -5 & 11 & -5 & 29 & 43 & 77 & -13 & 24 & 31 & 12 & 11 \\
\hline Financing & 95 & 31 & -93 & -6 & 11 & 19 & -67 & -60 & -62 & -66 & -122 \\
\hline Domestic financing (net) & 7 & -21 & -23 & -21 & 1 & 30 & 65 & -50 & -18 & 25 & 73 \\
\hline Banking system & 5 & -1 & -32 & -20 & 5 & 36 & 31 & -53 & -24 & 11 & 15 \\
\hline National Bank of Kazakhstan & -12 & -29 & -45 & -32 & -27 & 3 & 5 & -66 & -32 & -20 & 4 \\
\hline Commercial banks & 17 & 28 & 14 & 12 & 31 & 33 & 25 & 13 & 8 & 32 & 11 \\
\hline Non-bank financing & 3 & -20 & 9 & -2 & -4 & -6 & 34 & 3 & 6 & 14 & 58 \\
\hline Foreign financing, net & 51 & 30 & 29 & 0 & -2 & -3 & -56 & 5 & 4 & 8 & 8 \\
\hline Disbursements & 86 & 41 & 45 & 4 & 7 & 10 & 18 & 9 & 14 & 23 & 30 \\
\hline Amortization & -35 & -11 & -16 & -4 & -9 & -13 & -74 & -4 & -10 & -15 & -22 \\
\hline Privatization receipts & 37 & 22 & 82 & 18 & 18 & 19 & 19 & 31 & 58 & 59 & 59 \\
\hline National Fund of the Republic of Kazakhstan & 0 & 0 & -181 & -3 & -6 & -28 & -95 & -46 & -106 & -150 & -255 \\
\hline Other capital operations & 0 & 0 & 0 & 0 & 0 & 0 & 0 & 0 & 0 & -8 & -8 \\
\hline \multicolumn{12}{|l|}{ Memorandum items: } \\
\hline Nominal GDP & 2,016 & 2,600 & 3,251 & 746 & 1,666 & 2,754 & 3,776 & 933 & 2,015 & 3,270 & 4,450 \\
\hline
\end{tabular}

Source: Kazakhstani authorities, and Fund staff estimates.

$1 /$ Cumulative since the end of the previous year.

2/ Oil royalties, bonuses, and production sharing agreements.

3/ Includes a US\$210 million bonus payment from a large oil company in 2001.

4/ The large discrepancy in the third quarter of 2002 is related to the repayment of a $\$ 350$ million eurobond, in which funds were transferred from the banking system to an escrow account in the third quarter, while repayment to foreign creditors did not take place until the fourth quarter. 
Table 18b. Kazakhstan: General Government Fiscal Operations, 1999-2003 (In percent of GDP)

\begin{tabular}{|c|c|c|c|c|c|c|c|c|c|c|c|}
\hline & \multirow[t]{2}{*}{1999} & \multirow[t]{2}{*}{2000} & \multirow[t]{2}{*}{2001} & \multicolumn{4}{|c|}{$20021 /$} & \multicolumn{4}{|c|}{$20031 /$} \\
\hline & & & & Q1 & Q2 & Q3 & $\overline{\mathrm{Q} 4}$ & Q1 & Q2 & Q3 & Q4 \\
\hline Total revenues and grants & 17.5 & 21.7 & 25.7 & 23.7 & 22.9 & 22.0 & 22.5 & 29.0 & 27.1 & 25.2 & 26.3 \\
\hline Total revenues & 17.4 & 21.6 & 25.6 & 23.7 & 22.9 & 22.0 & 22.5 & 29.0 & 27.1 & 25.2 & 26.3 \\
\hline Oil revenues & 1.0 & 3.3 & 6.6 & 4.1 & 3.1 & 3.7 & 4.4 & 7.5 & 5.5 & 4.9 & 6.2 \\
\hline Non-oil revenues & 16.5 & 18.3 & 19.0 & 19.6 & 19.8 & 18.4 & 18.1 & 21.5 & 21.6 & 20.2 & 20.1 \\
\hline Current revenues & 17.4 & 21.5 & 25.4 & 23.6 & 22.8 & 21.8 & 22.2 & 28.9 & 27.0 & 24.9 & 26.0 \\
\hline Capital revenues & 0.0 & 0.1 & 0.3 & 0.1 & 0.1 & 0.2 & 0.3 & 0.1 & 0.1 & 0.3 & 0.3 \\
\hline Tax revenues & 16.0 & 20.0 & 22.3 & 22.5 & 21.1 & 20.4 & 21.0 & 27.9 & 24.0 & 22.7 & 24.1 \\
\hline Income tax & 4.5 & 8.3 & 9.4 & 9.3 & 8.0 & 7.9 & 8.5 & 13.6 & 10.0 & 9.5 & 10.0 \\
\hline Corporate income tax & 2.7 & 6.3 & 7.3 & 7.1 & 5.9 & 5.9 & 6.5 & 11.4 & 7.9 & 7.6 & 7.9 \\
\hline Oil revenues & 0.4 & 2.6 & 3.4 & 2.6 & 1.6 & 2.2 & 2.6 & 5.0 & 3.0 & 2.7 & 3.5 \\
\hline Non-oil revenues & 2.3 & 3.7 & 3.9 & 4.5 & 4.2 & 3.6 & 3.9 & 6.5 & 4.9 & 4.9 & 4.4 \\
\hline Personal income tax & 1.8 & 2.0 & 2.1 & 2.2 & 2.2 & 2.0 & 2.0 & 2.2 & 2.1 & 2.0 & 2.1 \\
\hline Social tax & 3.5 & 3.8 & 3.8 & 3.7 & 3.7 & 3.5 & 3.5 & 3.6 & 3.6 & 3.4 & 3.5 \\
\hline Property taxes & 1.2 & 1.0 & 1.0 & 1.1 & 1.1 & 0.9 & 0.9 & 1.0 & 0.9 & 0.9 & 0.9 \\
\hline Domestic taxes on goods, works and services & 6.2 & 6.2 & 7.2 & 7.3 & 7.2 & 7.0 & 6.8 & 8.5 & 8.2 & 7.7 & 8.5 \\
\hline Excises & 0.9 & 0.7 & 0.7 & 0.8 & 0.7 & 0.7 & 0.7 & 0.6 & 0.6 & 0.6 & 0.6 \\
\hline Receipts from use of natural resources & 0.5 & 0.8 & 1.5 & 1.3 & 1.1 & 1.1 & 1.4 & 2.4 & 2.5 & 2.1 & 2.7 \\
\hline Oil revenues 2/ & 0.2 & 0.5 & 1.3 & 0.7 & 0.5 & 0.6 & 0.9 & 1.7 & 1.9 & 1.6 & 1.9 \\
\hline Non-oil revenues & 0.3 & 0.4 & 0.2 & 0.5 & 0.6 & 0.5 & 0.5 & 0.7 & 0.6 & 0.5 & 0.8 \\
\hline Business and professional licences & 0.3 & 0.2 & 0.1 & 0.1 & 0.1 & 0.1 & 0.1 & 0.1 & 0.1 & 0.1 & 0.1 \\
\hline Taxes on international trade and external transactions & 0.6 & 0.7 & 0.8 & 0.9 & 1.0 & 1.0 & 1.0 & 1.0 & 1.0 & 1.0 & 1.0 \\
\hline Other taxes & 0.1 & 0.0 & 0.0 & 0.2 & 0.2 & 0.2 & 0.1 & 0.2 & 0.2 & 0.2 & 0.2 \\
\hline Non-tax revenues $3 /$ & 1.3 & 1.5 & 3.1 & 1.1 & 1.7 & 1.4 & 1.2 & 1.0 & 3.0 & 2.3 & 1.9 \\
\hline Income from capital transactions & 0.0 & 0.1 & 0.3 & 0.1 & 0.1 & 0.2 & 0.3 & 0.1 & 0.1 & 0.3 & 0.3 \\
\hline Grants & 0.1 & 0.1 & 0.0 & 0.0 & 0.0 & 0.0 & 0.0 & 0.0 & 0.0 & 0.0 & 0.0 \\
\hline Total expenditure and net lending & 22.7 & 22.5 & 23.0 & 18.9 & 21.0 & 19.9 & 21.0 & 19.9 & 22.5 & 22.8 & 23.3 \\
\hline Total expenditures & 21.8 & 21.8 & 22.3 & 18.6 & 20.4 & 19.3 & 20.5 & 19.4 & 21.8 & 21.4 & 22.2 \\
\hline Current expenditures & 20.5 & 20.2 & 19.5 & 16.8 & 18.1 & 16.6 & 17.2 & 16.6 & 17.8 & 16.8 & 17.2 \\
\hline Expenditures on goods and services & $\ldots$ & $\ldots$ & 12.4 & $\ldots$ & 10.8 & 10.1 & 10.7 & 10.3 & 11.2 & 10.7 & 10.7 \\
\hline Wages & $\ldots$ & $\ldots$ & 3.3 & $\ldots$ & 3.9 & 3.5 & 3.5 & 3.6 & 3.8 & 3.4 & 3.4 \\
\hline Employers' contributions & $\ldots$ & $\ldots$ & 0.5 & $\ldots$ & 0.6 & 0.5 & 0.5 & 0.5 & 0.5 & 0.5 & 0.5 \\
\hline Goods and services & $\ldots$ & $\ldots$ & 7.8 & $\ldots$ & 5.6 & 5.4 & 5.8 & 5.2 & 5.9 & 5.8 & 5.9 \\
\hline Other expenditures on goods and services & $\ldots$ & $\ldots$ & 0.8 & $\ldots$ & 0.8 & 0.8 & 0.9 & 0.9 & 1.0 & 1.0 & 1.0 \\
\hline Current transfers & $\ldots$ & $\ldots$ & 6.0 & $\ldots$ & 6.2 & 5.6 & 5.5 & 6.0 & 5.7 & 5.5 & 5.6 \\
\hline Capital expenditures & 1.4 & 1.6 & 2.8 & 1.7 & 2.3 & 2.7 & 3.3 & 2.8 & 4.0 & 4.6 & 5.0 \\
\hline Net lending & 0.9 & 0.7 & 0.7 & 0.3 & 0.6 & 0.6 & 0.5 & 0.6 & 0.7 & 1.4 & 1.1 \\
\hline Extending of credits; share participation & $\ldots$ & $\ldots$ & 1.0 & $\ldots$ & 0.9 & 0.8 & 0.8 & 0.8 & 1.0 & 1.7 & 1.5 \\
\hline Repayment of credits & $\ldots$ & $\ldots$ & 0.4 & $\cdots$ & 0.3 & 0.2 & 0.4 & 0.2 & 0.3 & 0.3 & 0.4 \\
\hline Overall budget balance & -5.0 & -0.8 & 2.7 & 4.8 & 1.9 & 2.1 & 1.4 & 9.0 & 4.6 & 2.4 & 3.0 \\
\hline Statistical discrepancy 4/ & -0.3 & 0.4 & -0.2 & 3.4 & 2.0 & 2.9 & -0.4 & 2.6 & 1.6 & 0.4 & 0.2 \\
\hline Financing & 4.7 & 1.2 & -2.9 & -1.3 & 0.2 & 0.8 & -1.8 & -6.4 & -3.1 & -2.0 & -2.7 \\
\hline Domestic financing (net) & 0.4 & -0.8 & -0.7 & -2.9 & 0.1 & 1.1 & 1.7 & -5.4 & -0.9 & 0.8 & 1.6 \\
\hline Banking system & 0.2 & 0.0 & -1.0 & -2.6 & 0.3 & 1.3 & 0.8 & -5.7 & -1.2 & 0.3 & 0.3 \\
\hline National Bank of Kazakhstan & -0.6 & -1.1 & -1.4 & -4.3 & -1.6 & 0.1 & 0.1 & -7.1 & -1.6 & -0.6 & 0.1 \\
\hline Commercial banks & 0.8 & 1.1 & 0.4 & 1.7 & 1.9 & 1.2 & 0.7 & 1.4 & 0.4 & 1.0 & 0.2 \\
\hline Non-bank financing & 0.1 & -0.8 & 0.3 & -0.2 & -0.2 & -0.2 & 0.9 & 0.3 & 0.3 & 0.4 & 1.3 \\
\hline Foreign financing, net & 2.6 & 1.2 & 0.9 & 0.0 & -0.1 & -0.1 & -1.5 & 0.6 & 0.2 & 0.2 & 0.2 \\
\hline Disbursements & 4.3 & 1.6 & 1.4 & 0.5 & 0.4 & 0.4 & 0.5 & 1.0 & 0.7 & 0.7 & 0.7 \\
\hline Amortization & -1.7 & -0.4 & -0.5 & -0.5 & -0.5 & -0.5 & -2.0 & -0.4 & -0.5 & -0.5 & -0.5 \\
\hline Privatization receipts & 1.8 & 0.8 & 2.5 & 2.4 & 1.1 & 0.7 & 0.5 & 3.3 & 2.9 & 1.8 & 1.3 \\
\hline National Fund of the Republic of Kazakhstan & 0.0 & 0.0 & -5.6 & -0.9 & -0.9 & -0.9 & -2.5 & -4.9 & -5.3 & -4.6 & -5.7 \\
\hline Other capital operations & $\ldots$ & 0.0 & 0.0 & 0.0 & 0.0 & 0.0 & 0.0 & 0.0 & 0.0 & -0.2 & -0.2 \\
\hline \multicolumn{12}{|l|}{ Memorandum items: } \\
\hline Nominal GDP (in billions of tenge) & 2,016 & 2,600 & 3,251 & 746 & 1,666 & 2,754 & 3,776 & 933 & 2,015 & 3,270 & 4,450 \\
\hline
\end{tabular}

Source: Kazakhstani authorities, Fund staff estimates.

$1 /$ Cumulative since the end of the previous year.

$2 /$ Oil royalties, bonuses, and production sharing agreements.

3 / Includes a US\$210 million bonus payment from a large oil company in 2001

4/ The large discrepancy in the third quarter of 2002 is related to the repayment of a $\$ 350$ million eurobond, in which funds were transferred from the banking system to an escrow account in the third quarter, while repayment to foreign creditors did not take place until the fourth quarter. 
Table 19a. Kazakhstan: Central Government Fiscal Operations, 1999-2003 (In billions of tenge)

\begin{tabular}{|c|c|c|c|c|c|c|c|c|c|c|c|}
\hline & \multirow[t]{2}{*}{1999} & \multirow[t]{2}{*}{2000} & \multirow[t]{2}{*}{2001} & \multicolumn{4}{|c|}{$20021 /$} & \multicolumn{4}{|c|}{$20031 /$} \\
\hline & & & & Q1 & Q2 & Q3 & $\mathrm{Q} 4$ & Q1 & Q2 & Q3 & Q4 \\
\hline Total revenues and grants & 172 & 297 & 497 & 111 & 239 & 389 & 545 & 193 & 381 & 568 & 814 \\
\hline Total revenues & 169 & 294 & 497 & 111 & 239 & 389 & 545 & 193 & 381 & 568 & 814 \\
\hline Oil revenues & 8 & 46 & 203 & 28 & 44 & 89 & 149 & 67 & 106 & 152 & 262 \\
\hline Non-oil revenues & 161 & 249 & 294 & 83 & 194 & 300 & 397 & 126 & 275 & 416 & 552 \\
\hline Current revenues & 168 & 292 & 491 & 111 & 238 & 386 & 540 & 193 & 380 & 563 & 807 \\
\hline Capital revenues & 1 & 2 & 6 & 0 & 0 & 3 & 6 & 0 & 1 & 4 & 6 \\
\hline Tax revenues & 152 & 263 & 402 & 104 & 212 & 350 & 498 & 185 & 322 & 472 & 727 \\
\hline Income tax & 28 & 83 & 158 & 53 & 98 & 162 & 244 & 107 & 159 & 224 & 352 \\
\hline Corporate income tax & 27 & 82 & 158 & 53 & 98 & 162 & 244 & 107 & 159 & 211 & 273 \\
\hline Oil revenues & 4 & 34 & 111 & 20 & 27 & 62 & 97 & 47 & 60 & 89 & 157 \\
\hline Non-oil revenues & 24 & 48 & 48 & 33 & 70 & 100 & 147 & 60 & 99 & 122 & 116 \\
\hline Personal income tax & 1 & 1 & 0 & 0 & 0 & 0 & 0 & 0 & 0 & 0 & 0 \\
\hline Social tax & 2 & 20 & 0 & 0 & 0 & 0 & 0 & 0 & 0 & 0 & 0 \\
\hline Property taxes & 1 & 1 & 0 & 0 & 0 & 0 & 0 & 0 & 0 & 0 & 0 \\
\hline Domestic taxes on goods, works and services & 108 & 141 & 216 & 44 & 97 & 159 & 213 & 68 & 142 & 215 & 331 \\
\hline VAT & 81 & 103 & 151 & 36 & 83 & 134 & 167 & 50 & 98 & 154 & 222 \\
\hline Excises & 15 & 15 & 18 & 1 & 1 & 3 & 4 & 1 & 3 & 4 & 6 \\
\hline Receipts from the use of natural resources & 9 & 21 & 47 & 7 & 12 & 22 & 42 & 17 & 41 & 56 & 102 \\
\hline Oil revenues $2 /$ & 4 & $\ldots$ & 41 & 6 & 9 & 17 & 35 & 16 & 38 & 47 & 79 \\
\hline Non-oil revenues & 5 & $\ldots$ & 6 & 1 & 3 & 5 & 7 & 1 & 3 & 9 & 23 \\
\hline Business and professional licences & 3 & 1 & 1 & 0 & 0 & 0 & 1 & 0 & 0 & 0 & 0 \\
\hline Taxes on international trade and external transactions & 11 & 18 & 26 & 7 & 16 & 26 & 39 & 9 & 20 & 31 & 42 \\
\hline Other taxes & 1 & 1 & 1 & 1 & 2 & 2 & 2 & 0 & 1 & 2 & 2 \\
\hline Non-tax revenues $3 /$ & 16 & 29 & 89 & 7 & 26 & 36 & 42 & 8 & 58 & 91 & 80 \\
\hline Income from capital transactions & 1 & 2 & 6 & 0 & 0 & 3 & 6 & 0 & 1 & 4 & 6 \\
\hline Grants & 2 & 3 & 0 & 0 & 0 & 0 & 0 & 0 & 0 & 0 & 0 \\
\hline Total expenditures and net lending and transfers & 281 & 327 & 406 & 92 & 215 & 337 & 484 & 127 & 303 & 506 & 688 \\
\hline Total expenditures and net lending & 288 & 339 & 450 & 85 & 208 & 327 & 469 & 119 & 288 & 487 & 661 \\
\hline Total expenditures & 266 & 322 & 435 & 79 & 197 & 311 & 453 & 112 & 275 & 442 & 610 \\
\hline Current expenditures & 249 & 310 & 376 & $\ldots$ & 181 & 275 & 390 & 91 & 213 & 333 & 461 \\
\hline Expenditures on goods and services & $\ldots$ & $\ldots$ & 180 & $\ldots$ & 78 & 125 & 183 & 41 & 102 & 162 & 219 \\
\hline Wages & $\ldots$ & $\ldots$ & 36 & $\ldots$ & 19 & 30 & 43 & 10 & 21 & 34 & 46 \\
\hline Employers' contributions & $\ldots$ & $\ldots$ & 4 & $\ldots$ & 2 & 3 & 4 & 1 & 2 & 3 & 4 \\
\hline Goods and services & $\ldots$ & $\ldots$ & 117 & $\ldots$ & 46 & 72 & 107 & 24 & 60 & 94 & 126 \\
\hline Other current expenditures & $\ldots$ & $\ldots$ & 23 & $\ldots$ & 12 & 20 & 30 & 5 & 15 & 23 & 31 \\
\hline Interest & 21 & 35 & 37 & $\ldots$ & 19 & 24 & 38 & 3 & 17 & 21 & 34 \\
\hline Domestic & $\ldots$ & $\ldots$ & 8 & $\ldots$ & 4 & 5 & 9 & 2 & 5 & 7 & 11 \\
\hline Foreign & $\ldots$ & $\ldots$ & 29 & $\ldots$ & 15 & 18 & 29 & 1 & 12 & 14 & 23 \\
\hline Current transfers & $\ldots$ & $\ldots$ & 159 & $\ldots$ & 84 & 126 & 169 & 46 & 94 & 150 & 208 \\
\hline Capital expenditures & 17 & 13 & 59 & $\ldots$ & 16 & 36 & 63 & 21 & 62 & 109 & 149 \\
\hline of which: Capital transfers to other levels of government & $\ldots$ & $\ldots$ & 8 & $\ldots$ & 5 & 10 & 13 & 6 & 21 & 33 & 38 \\
\hline Net lending & 21 & 17 & 15 & 6 & 11 & 16 & 16 & 8 & 13 & 45 & 51 \\
\hline Extending of credits; share participation & & & 27 & 8 & 15 & 20 & 27 & 10 & 19 & 52 & 66 \\
\hline Repayment of credits & & & 12 & 2 & 4 & 4 & 11 & 2 & 6 & 7 & 15 \\
\hline Net transfers to other levels of government & -7 & -12 & -44 & 7 & 7 & 10 & 14 & 7 & 15 & 19 & 27 \\
\hline Transfers from other levels of government & 37 & 54 & 84 & 12 & 26 & 39 & 49 & 15 & 32 & 48 & 58 \\
\hline Transfers to other levels of government & 30 & 42 & 40 & 19 & 33 & 50 & 64 & 22 & 47 & 67 & 85 \\
\hline Capital transfer from other levels of government & $\ldots$ & $\ldots$ & $\ldots$ & $\ldots$ & 0 & 0 & 0 & 0 & 0 & 0 & 0 \\
\hline Overall budget balance (cash) & -109 & -30 & 92 & 19 & 24 & 52 & 62 & 67 & 78 & 62 & 139 \\
\hline Statistical discrepancy 4/ & 0 & 0 & -4 & 6 & 25 & 74 & 6 & 4 & 11 & -8 & 14 \\
\hline Financing & 109 & 30 & -96 & -13 & 2 & 22 & -56 & -63 & -67 & -69 & -125 \\
\hline Domestic financing (net) & 24 & -21 & -23 & -24 & 1 & 30 & 34 & -53 & -22 & 22 & 72 \\
\hline Banking system & $\ldots$ & $\ldots$ & -32 & -22 & 4 & 36 & 30 & -56 & -28 & 9 & 14 \\
\hline National Bank of Kazakhstan & $\ldots$ & $\ldots$ & -45 & -32 & -27 & 3 & 3 & -66 & -32 & -20 & 4 \\
\hline Commercial banks & $\ldots$ & $\ldots$ & 14 & 10 & 31 & 33 & 26 & 10 & 4 & 29 & 10 \\
\hline Non-bank financing & & & 9 & -2 & -4 & -6 & 4 & 3 & 6 & 14 & 58 \\
\hline Foreign financing, net & 51 & 30 & 29 & 0 & -2 & -3 & -56 & 5 & 4 & 8 & 8 \\
\hline Disbursements & $\ldots$ & $\ldots$ & $\ldots$ & 4 & 7 & 10 & 18 & 9 & 14 & 23 & $\ldots$ \\
\hline Amortization & $\ldots$ & $\ldots$ & $\ldots$ & 4 & 9 & 13 & 74 & 4 & 10 & 15 & $\ldots$ \\
\hline Privatization receipts & 34 & 21 & 81 & 18 & 18 & 18 & 18 & 31 & 57 & 58 & 58 \\
\hline National Fund of the Republic of Kazakhstan (NFRK) & 0 & 0 & -182 & -6 & -15 & -24 & -53 & -46 & -106 & -150 & -255 \\
\hline Other capital operations & 0 & 0 & 0 & 0 & 0 & 0 & 0 & 0 & 0 & -8 & -8 \\
\hline \multicolumn{12}{|l|}{ Memorandum items: } \\
\hline Nominal GDP & 2,016 & 2,600 & 3,251 & 746 & 1,666 & 2,754 & 3,776 & 933 & 2,015 & 3,270 & 4,450 \\
\hline
\end{tabular}

Source: Kazakhstani authorities, Fund staff estimates.

$1 /$ Cumulative since the end of the previous year.

2/ Oil royalties, bonuses, and production sharing agreements.

3/ Includes a US $\$ 210$ million bonus payment from a large oil company in 2001.

4/ The large discrepancy in the third quarter of 2002 is related to the repayment of a $\$ 350$ million eurobond, in which funds were transferred from the banking system to an escrow account in the third quarter, while repayment to foreign creditors did not take place until the fourth quarter. 
Table 19b. Kazakhstan: Central Government Fiscal Operations, 1999-2003 (In percent of GDP)

\begin{tabular}{|c|c|c|c|c|c|c|c|c|c|c|c|}
\hline & \multirow[t]{2}{*}{1999} & \multirow[t]{2}{*}{2000} & \multirow{2}{*}{2001} & \multicolumn{4}{|c|}{$20021 /$} & \multicolumn{4}{|c|}{$20031 /$} \\
\hline & & & & Q1 & Q2 & Q3 & $\overline{\mathrm{Q} 4}$ & Q1 & Q2 & Q3 & $\mathrm{Q} 4$ \\
\hline Total revenues and grants & 8.5 & 11.4 & 15.3 & 14.9 & 14.3 & 14.1 & 14.4 & 20.7 & 18.9 & 17.4 & 18.3 \\
\hline Total revenues & 8.4 & 11.3 & 15.3 & 14.9 & 14.3 & 14.1 & 14.4 & 20.7 & 18.9 & 17.4 & 18.3 \\
\hline Oil revenues & 0.4 & 1.8 & 6.2 & 3.7 & 2.7 & 3.2 & 3.9 & 7.2 & 5.3 & 4.6 & 5.9 \\
\hline Non-oil revenues & 8.0 & 9.6 & 9.0 & 11.2 & 11.7 & 10.9 & 10.5 & 13.5 & 13.6 & 12.7 & 12.4 \\
\hline Current revenues & 8.3 & 11.2 & 15.1 & 14.9 & 14.3 & 14.0 & 14.3 & 20.7 & 18.9 & 17.2 & 18.1 \\
\hline Capital revenues & 0.0 & 0.1 & 0.2 & 0.0 & 0.0 & 0.1 & 0.2 & 0.0 & 0.0 & 0.1 & 0.1 \\
\hline Tax revenues & 7.5 & 10.1 & 12.4 & 14.0 & 12.7 & 12.7 & 13.2 & 19.8 & 16.0 & 14.4 & 16.3 \\
\hline Income tax & 1.4 & 3.2 & 4.9 & 7.1 & 5.9 & 5.9 & 6.5 & 11.4 & 7.9 & 6.9 & 7.9 \\
\hline Corporate income tax & 1.4 & 3.1 & 4.9 & 7.1 & 5.9 & 5.9 & 6.5 & 11.4 & 7.9 & 6.4 & 6.1 \\
\hline Oil revenues & 0.2 & 1.3 & 3.4 & 2.6 & 1.6 & 2.2 & 2.6 & 5.0 & 3.0 & 2.7 & 3.5 \\
\hline Non-oil revenues & 1.2 & 1.9 & 1.5 & 4.5 & 4.2 & 3.6 & 3.9 & 6.4 & 4.9 & 3.7 & 2.6 \\
\hline Personal income tax & 0.0 & 0.0 & 0.0 & 0.0 & 0.0 & 0.0 & 0.0 & 0.0 & 0.0 & 0.0 & 0.0 \\
\hline Social tax & 0.1 & 0.8 & 0.0 & 0.0 & 0.0 & 0.0 & 0.0 & 0.0 & 0.0 & 0.0 & 0.0 \\
\hline Property taxes & 0.0 & 0.0 & 0.0 & 0.0 & 0.0 & 0.0 & 0.0 & 0.0 & 0.0 & 0.0 & 0.0 \\
\hline Domestic taxes on goods, works and services & 5.4 & 5.4 & 6.7 & 5.8 & 5.8 & 5.8 & 5.6 & 7.3 & 7.0 & 6.6 & 7.4 \\
\hline VAT & 4.0 & 4.0 & 4.6 & 4.8 & 5.0 & 4.9 & 4.4 & 5.4 & 4.8 & 4.7 & 5.0 \\
\hline Excises & 0.7 & 0.6 & 0.5 & 0.1 & 0.1 & 0.1 & 0.1 & 0.1 & 0.1 & 0.1 & 0.1 \\
\hline Receipts from the use of natural resources & 0.5 & 0.8 & 1.5 & 0.9 & 0.7 & 0.8 & 1.1 & 1.8 & 2.0 & 1.7 & 2.3 \\
\hline Oil revenues $2 /$ & 0.2 & $\ldots$ & 1.3 & 0.7 & 0.5 & 0.6 & 0.9 & 1.7 & 1.9 & 1.4 & 1.8 \\
\hline Non-oil revenues & 0.3 & $\ldots$ & 0.2 & 0.2 & 0.2 & 0.2 & 0.2 & 0.1 & 0.2 & 0.3 & 0.5 \\
\hline Business and professional licences & 0.1 & 0.0 & 0.0 & 0.0 & 0.0 & 0.0 & 0.0 & 0.0 & 0.0 & 0.0 & 0.0 \\
\hline Taxes on international trade and external transactions & 0.6 & 0.7 & 0.8 & 0.9 & 1.0 & 1.0 & 1.0 & 1.0 & 1.0 & 1.0 & 1.0 \\
\hline Other taxes & 0.0 & 0.0 & 0.0 & 0.1 & 0.1 & 0.1 & 0.1 & -0.1 & 0.0 & 0.1 & 0.0 \\
\hline Non-tax revenues $3 /$ & 0.8 & 1.1 & 2.7 & 1.0 & 1.6 & 1.3 & 1.1 & 0.9 & 2.9 & 2.8 & 1.8 \\
\hline Income from capital transactions & 0.0 & 0.1 & 0.2 & 0.0 & 0.0 & 0.1 & 0.2 & 0.0 & 0.0 & 0.1 & 0.1 \\
\hline Grants & 0.1 & 0.1 & 0.0 & 0.0 & 0.0 & 0.0 & 0.0 & 0.0 & 0.0 & 0.0 & 0.0 \\
\hline Total expenditures and net lending and transfers & 13.9 & 12.6 & 12.5 & 12.4 & 12.9 & 12.2 & 12.8 & 13.6 & 15.0 & 15.5 & 15.5 \\
\hline Total expenditures and net lending & 14.3 & 13.0 & 13.8 & 11.4 & 12.5 & 11.9 & 12.4 & 12.7 & 14.3 & 14.9 & 14.9 \\
\hline Total expenditures & 13.2 & 12.4 & 13.4 & 10.7 & 11.8 & 11.3 & 12.0 & 12.0 & 13.6 & 13.5 & 13.7 \\
\hline Current expenditures & 12.4 & 11.9 & 11.6 & $\ldots$ & 10.9 & 10.0 & 10.3 & 9.7 & 10.6 & 10.2 & 10.4 \\
\hline Expenditures on goods and services & $\ldots$ & $\ldots$ & 5.5 & $\ldots$ & 4.7 & 4.5 & 4.8 & 4.4 & 5.1 & 5.0 & 4.9 \\
\hline Wages & $\ldots$ & $\ldots$ & 1.1 & $\ldots$ & 1.1 & 1.1 & 1.1 & 1.0 & 1.1 & 1.0 & 1.0 \\
\hline Employers' contributions & $\ldots$ & $\ldots$ & 0.1 & $\ldots$ & 0.1 & 0.1 & 0.1 & 0.1 & 0.1 & 0.1 & 0.1 \\
\hline Goods and services & $\ldots$ & $\ldots$ & 3.6 & $\ldots$ & 2.7 & 2.6 & 2.8 & 2.5 & 3.0 & 2.9 & 2.8 \\
\hline Other current expenditures & $\ldots$ & $\ldots$ & 0.7 & $\ldots$ & 0.7 & 0.7 & 0.8 & 0.5 & 0.7 & 0.7 & 0.7 \\
\hline Interest & 1.1 & 1.3 & 1.1 & $\ldots$ & 1.1 & 0.9 & 1.0 & 0.4 & 0.9 & 0.6 & 0.8 \\
\hline Domestic & $\ldots$ & $\ldots$ & 0.3 & $\ldots$ & 0.2 & 0.2 & 0.2 & 0.2 & 0.3 & 0.2 & 0.2 \\
\hline Foreign & $\ldots$ & $\ldots$ & 0.9 & $\ldots$ & 0.9 & 0.7 & 0.8 & 0.2 & 0.6 & 0.4 & 0.5 \\
\hline Current transfers & $\ldots$ & $\ldots$ & 4.9 & $\ldots$ & 5.0 & 4.6 & 4.5 & 4.9 & 4.7 & 4.6 & 4.7 \\
\hline Capital expenditures & 0.8 & 0.5 & 1.8 & $\ldots$ & 1.0 & 1.3 & 1.7 & 2.2 & 3.1 & 3.3 & 3.3 \\
\hline of which: Capital transfers to other levels of government & $\ldots$ & $\ldots$ & 0.2 & $\ldots$ & 0.3 & 0.4 & 0.3 & 0.6 & 1.1 & 1.0 & 0.9 \\
\hline Net lending & 1.1 & 0.7 & 0.5 & 0.8 & 0.7 & 0.6 & 0.4 & 0.9 & 0.7 & 1.4 & 1.1 \\
\hline Extending of credits; share participation & 0.0 & 0.0 & 0.8 & 1.1 & 0.9 & 0.7 & 0.7 & 1.0 & 0.9 & 1.6 & 1.5 \\
\hline Repayment of credits & 0.0 & 0.0 & 0.4 & 0.3 & 0.2 & 0.2 & 0.3 & 0.2 & 0.3 & 0.2 & 0.3 \\
\hline Net transfers to other levels of government & -0.3 & -0.5 & -1.4 & 0.9 & 0.4 & 0.4 & 0.4 & 0.8 & 0.8 & 0.6 & 0.6 \\
\hline Transfers from other levels of government & 1.8 & 2.1 & 2.6 & 1.7 & 1.6 & 1.4 & 1.3 & 1.6 & 1.6 & 1.5 & 1.3 \\
\hline Transfers to other levels of government & 1.5 & 1.6 & 1.2 & 2.6 & 2.0 & 1.8 & 1.7 & 2.4 & 2.3 & 2.0 & 1.9 \\
\hline Capital transfer from other levels of government & $\ldots$ & $\ldots$. & $\ldots$. & $\ldots$ & 0.0 & 0.0 & 0.0 & 0.0 & 0.0 & 0.0 & 0.0 \\
\hline Overall budget balance (cash) & -5.4 & -1.2 & 2.8 & 2.5 & 1.4 & 1.9 & 1.6 & 7.2 & 3.9 & 1.9 & 3.1 \\
\hline Statistical discrepancy 4 / & 0.0 & 0.0 & -0.1 & 0.8 & 1.5 & 2.7 & 0.1 & 0.4 & 0.5 & -0.2 & 0.3 \\
\hline Financing & 5.4 & 1.2 & -2.9 & -1.7 & 0.1 & 0.8 & -1.5 & -6.7 & -3.3 & -2.1 & -2.8 \\
\hline Domestic financing (net) & 1.2 & -0.8 & -0.7 & -3.2 & 0.0 & 1.1 & 0.9 & -5.7 & -1.1 & 0.7 & 1.6 \\
\hline Banking system & $\ldots$ & $\ldots$ & -1.0 & -3.0 & 0.2 & 1.3 & 0.8 & -6.0 & -1.4 & 0.3 & 0.3 \\
\hline National Bank of Kazakhstan & $\ldots$ & $\ldots$ & -1.4 & -4.3 & -1.6 & 0.1 & 0.1 & -7.1 & -1.6 & -0.6 & 0.1 \\
\hline Commercial banks & & $\ldots$ & 0.4 & 1.4 & 1.8 & 1.2 & 0.7 & 1.1 & 0.2 & 0.9 & 0.2 \\
\hline Non-bank financing & 0.0 & 0.0 & 0.3 & -0.2 & -0.2 & -0.2 & 0.1 & 0.3 & 0.3 & 0.4 & 1.3 \\
\hline Foreign financing, net & 2.6 & 1.2 & 0.9 & 0.0 & -0.1 & -0.1 & -1.5 & 0.5 & 0.2 & 0.2 & 0.2 \\
\hline Disbursements & $\ldots$ & $\ldots$ & $\ldots$ & 0.5 & 0.4 & 0.4 & 0.5 & 1.0 & 0.7 & 0.7 & $\ldots$ \\
\hline Amortization & & . & $\ldots$ & 0.5 & 0.5 & 0.5 & 2.0 & 0.4 & 0.5 & 0.5 & $\ldots$ \\
\hline Privatization receipts & 1.7 & 0.8 & 2.5 & 2.4 & 1.1 & 0.7 & 0.5 & 3.3 & 2.8 & 1.8 & 1.3 \\
\hline National Fund of the Republic of Kazakhstan (NFRK) & 0.0 & 0.0 & -5.6 & -0.9 & -0.9 & -0.9 & -1.4 & -4.9 & -5.3 & -4.6 & -5.7 \\
\hline Other capital operations & $\ldots$ & 0.0 & 0.0 & 0.0 & 0.0 & 0.0 & 0.0 & 0.0 & 0.0 & -0.2 & -0.2 \\
\hline \multicolumn{12}{|l|}{ Memorandum items: } \\
\hline Nominal GDP (in billions of tenge) & 2,016 & 2,600 & 3,251 & 746 & 1,666 & 2,754 & 3,776 & 933 & 2,041 & 3,271 & 4,450 \\
\hline Compensation flowing from the NFRK for stabilization & $\ldots$ & $\ldots$ & 0.1 & 0.0 & 0.1 & 0.1 & 0.1 & 0.0 & 0.0 & 0.0 & 0.0 \\
\hline
\end{tabular}

Source: Kazakhstani authorities, Fund staff estimates.

1/ Cumulative since the end of the previous year.

2/ Oil royalties, bonuses, and production sharing agreements

3/ Includes a US\$210 million bonus payment from a large oil company in 2001.

4/ The large discrepancy in the third quarter of 2002 is related to the repayment of a $\$ 350$ million eurobond, in which funds were transferred from the banking system to an escrow account in the third quarter, while repayment to foreign creditors did not take place until the fourth quarter. 
Table 20a. Kazakhstan: Local Government Fiscal Operations, 1999-2003 (In billions of tenge)

\begin{tabular}{|c|c|c|c|c|c|c|c|c|c|c|c|}
\hline & \multirow[t]{2}{*}{1999} & \multirow[t]{2}{*}{2000} & \multirow[t]{2}{*}{2001} & \multicolumn{4}{|c|}{$20021 /$} & \multicolumn{4}{|c|}{$20031 /$} \\
\hline & & & & Q1 & Q2 & Q3 & $\overline{\mathrm{Q} 4}$ & $\mathrm{Q1}$ & Q2 & Q3 & $\mathrm{Q4}$ \\
\hline Total revenues and grants & 180 & 268 & 337 & 65 & 143 & 218 & 302 & 77 & 166 & 256 & 356 \\
\hline Total revenues & 182 & 268 & 336 & 65 & 143 & 218 & 302 & 77 & 166 & 256 & 356 \\
\hline Oil revenues & 11 & 40 & 12 & 3 & 7 & 12 & 16 & 3 & 6 & 9 & 13 \\
\hline Non-oil revenues & 171 & 227 & 324 & 63 & 135 & 206 & 286 & 74 & 160 & 247 & 343 \\
\hline Current revenues & 182 & 267 & 334 & 65 & 141 & 215 & 299 & 76 & 164 & 251 & 349 \\
\hline Capital revenues & 0 & 0 & 3 & 1 & 2 & 3 & 4 & 1 & 2 & 4 & 6 \\
\hline Tax revenues & 171 & 258 & 322 & 64 & 139 & 212 & 295 & 76 & 161 & 247 & 344 \\
\hline Income tax & $\ldots$ & $\ldots$ & 147 & 16 & 36 & 56 & 77 & 20 & 43 & 66 & 93 \\
\hline Corporate income tax & $\ldots$ & $\ldots$ & 78 & 0 & 0 & 0 & 0 & 0 & 0 & 0 & 0 \\
\hline Oil revenues & $\ldots$ & $\ldots$ & 0 & 0 & 0 & 0 & 0 & 0 & 0 & 0 & 0 \\
\hline Non-oil revenues & $\ldots$ & $\ldots$ & 78 & 0 & 0 & 0 & 0 & 0 & 0 & 0 & 0 \\
\hline Personal income tax & $\ldots$ & $\ldots$ & 69 & 16 & 36 & 56 & 77 & 20 & 43 & 66 & 93 \\
\hline Social tax & $\ldots$ & $\ldots$ & 124 & 28 & 61 & 95 & 134 & 34 & 72 & 111 & 158 \\
\hline Property taxes & $\ldots$ & $\ldots$ & 33 & 8 & 18 & 26 & 35 & 9 & 19 & 29 & 38 \\
\hline Domestic taxes on goods, works and services & $\ldots$ & $\ldots$ & 18 & 11 & 23 & 33 & 45 & 11 & 24 & 37 & 49 \\
\hline VAT & $\ldots$ & $\ldots$ & 9 & 2 & 5 & 6 & 10 & 2 & 4 & 7 & 9 \\
\hline Excises & $\begin{array}{l}\cdots \\
\ldots\end{array}$ & $\cdots$ & 4 & 5 & 11 & 16 & 21 & 4 & 10 & 16 & 21 \\
\hline Receipts from the use of natural resources & $\ldots$ & $\ldots$ & 1 & 3 & 6 & 9 & 12 & 5 & 9 & 12 & 16 \\
\hline Oil revenues 2/ & $\ldots$ & $\ldots$ & 0 & 0 & 0 & 0 & 0 & 0 & 0 & 0 & 0 \\
\hline Non-oil revenues & $\ldots$ & $\ldots$ & 1 & 3 & 6 & 9 & 12 & 5 & 9 & 12 & 16 \\
\hline Business and professional licences & $\ldots$ & $\ldots$ & 4 & 0 & 1 & 2 & 2 & 1 & 1 & 2 & 3 \\
\hline Taxes on international trade and external transactions & $\ldots$ & $\ldots$ & 0 & 0 & 0 & 0 & 0 & 0 & 0 & 0 & 0 \\
\hline Other taxes & $\ldots$ & $\ldots$ & 0 & 1 & 2 & 2 & 3 & 1 & 3 & 4 & 5 \\
\hline Non-tax revenues & 11 & 10 & 11 & 1 & 2 & 3 & 4 & 1 & 3 & 4 & 6 \\
\hline Income from capital transactions & 0 & 0 & 3 & 1 & 2 & 3 & 4 & 1 & 2 & 4 & 6 \\
\hline Grants & -2 & 0 & 0 & 0 & 0 & 0 & 0 & 0 & 0 & 0 & 0 \\
\hline Total expenditures and net lending and transfers & 171 & 257 & 340 & 68 & 139 & 216 & 310 & 63 & 151 & 244 & 354 \\
\hline Total expenditures and net lending & 165 & 245 & 304 & $\ldots$ & 152 & 236 & 338 & 76 & 187 & 296 & 419 \\
\hline Total expenditures & 169 & 245 & 298 & $\ldots$ & 148 & 230 & 336 & 75 & 183 & 292 & 414 \\
\hline Current expenditures & 158 & 215 & 258 & $\ldots$ & 121 & 183 & 259 & 64 & 145 & 218 & 302 \\
\hline Expenditures on goods and services & $\ldots$ & $\ldots$ & 222 & $\ldots$ & 102 & 153 & 219 & 54 & 124 & 186 & 259 \\
\hline Wages & $\ldots$ & $\ldots$ & 70 & $\ldots$ & 46 & 65 & 91 & 24 & 54 & 77 & 104 \\
\hline Employers' contributions & $\ldots$ & $\ldots$ & 13 & $\ldots$ & 8 & 11 & 15 & 4 & 9 & 13 & 17 \\
\hline Goods and services & $\ldots$ & $\ldots$ & 137 & $\ldots$ & 47 & 76 & 111 & 26 & 59 & 94 & 134 \\
\hline Other current expenditures & $\ldots$ & $\ldots$ & 3 & $\ldots$ & 1 & 2 & 3 & 1 & 2 & 3 & 4 \\
\hline Interest & $\ddot{0}$ & 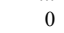 & 1 & $\cdots$ & 0 & 1 & 1 & 0 & 0 & 1 & 1 \\
\hline Domestic & $\ldots$ & $\ldots$ & 1 & $\ldots$ & 0 & 1 & 1 & 0 & 0 & 1 & 1 \\
\hline Foreign & $\ldots$ & $\ldots$ & 0 & $\ldots$ & 0 & 0 & 0 & 0 & 0 & 0 & 0 \\
\hline Current transfers & $\ldots$ & $\ldots$ & 35 & $\ldots$ & 19 & 29 & 39 & 10 & 20 & 31 & 42 \\
\hline Capital expenditures & 11 & 30 & 41 & $\ldots$ & 27 & 48 & 76 & 11 & 38 & 74 & 112 \\
\hline of which: Capital transfers to other levels of government & $\ldots$ & $\ldots$ & $\ldots$ & $\ldots$ & 0 & 0 & 0 & & & & \\
\hline Net lending & -4 & 0 & 6 & $\ldots$ & 4 & 6 & 2 & 1 & 4 & 4 & 5 \\
\hline Extending of credits; share participation & $\ldots$ & $\ldots$ & 11 & $\cdots$ & 5 & 8 & 9 & 2 & 6 & 7 & 7 \\
\hline Repayment of credits & $\ldots$ & $\ldots$ & 6 & $\ldots$ & 1 & 2 & 7 & 1 & 1 & 3 & 2 \\
\hline Net transfers to other levels of government & 7 & 12 & 36 & $\ldots$ & -12 & -20 & -27 & -13 & -36 & -52 & -65 \\
\hline Transfers from other levels of government & 30 & 42 & 40 & $\ldots$ & 33 & 50 & 64 & 22 & 47 & 67 & 85 \\
\hline Transfers to other levels of government & 37 & 54 & 84 & $\cdots$ & 26 & 39 & 49 & 15 & 32 & 48 & 58 \\
\hline Capital transfer from other levels of government & $\ldots$ & $\ldots$ & 8 & $\ldots$ & 5 & 10 & 13 & 6 & 21 & 33 & 38 \\
\hline Overall budget balance & 9 & 11 & -4 & -3 & 3 & 2 & -8 & 14 & 15 & 12 & 2 \\
\hline Statistical discrepancy & 0 & 11 & -3 & $\ldots$ & 4 & 2 & -9 & 17 & 20 & 16 & 2 \\
\hline Financing & -9 & 1 & 1 & 3 & 1 & 0 & -1 & 3 & 5 & 4 & 0 \\
\hline Domestic financing (net) & -11 & 0 & 0 & 2 & 1 & 0 & -1 & 3 & 4 & 3 & -1 \\
\hline Banking system & $\ldots$ & $\ldots$ & 0 & 2 & 1 & 0 & -1 & 3 & 4 & 3 & 1 \\
\hline National Bank of Kazakhstan & $\ldots$ & $\ldots$ & 0 & 0 & 0 & 0 & 0 & 0 & 0 & 0 & 0 \\
\hline Commercial banks & $\ldots$ & $\ldots$ & 0 & 2 & 1 & 0 & -1 & 3 & 4 & 3 & 1 \\
\hline Non-bank financing & $\cdots$ & $\cdots$ & 0 & 0 & 0 & 0 & 0 & 0 & 0 & 0 & 0 \\
\hline Foreign financing, net & 0 & 0 & 0 & 0 & 0 & 0 & 0 & 0 & 0 & 0 & 0 \\
\hline Disbursements & $\ldots$ & $\ldots$ & $\ldots$ & $\ldots$ & $\ldots$ & $\ldots$ & $\ldots$ & $\ldots$ & $\ldots$ & $\ldots$ & $\ldots$ \\
\hline Amortization & $\ldots$ & $\ldots$ & $\ldots$ & $\ldots$ & $\ldots$ & $\ldots$ & $\ldots$ & $\ldots$ & $\ldots$ & $\ldots$ & $\ldots$ \\
\hline Privatization receipts & 3 & 1 & 1 & 0 & 0 & 0 & 0 & 0 & 1 & 1 & 1 \\
\hline National Fund of the Republic of Kazakhstan (NFRK) & $\ldots$ & $\ldots$ & $\ldots$ & $\ldots$ & $\ldots$ & $\ldots$ & $\ldots$ & .. &.. & .. & .. \\
\hline \multicolumn{12}{|l|}{ Memorandum items: } \\
\hline Nominal GDP & 2,016 & 2,600 & 3,251 & 746 & 1,666 & 2,754 & 3,776 & 933 & 2,041 & 3,271 & 4,450 \\
\hline Compensation flowing from the NFRK for stabilization 2/ & $\ldots$ & $\ldots$ & 2.8 & 0.0 & 0.0 & 0.0 & 0.0 & 0.0 & 0.0 & 0.0 & 0.0 \\
\hline
\end{tabular}

Source: Kazakhstani authorities, Fund staff estimates.

1 / Cumulative since the end of the previous year.

2/ Oil royalties, bonuses, and production sharing agreements. 
Table 20b. Kazakhstan: Local Government Fiscal Operations, 1999-2003 (In percent of GDP)

\begin{tabular}{|c|c|c|c|c|c|c|c|c|c|c|c|}
\hline & \multirow[t]{2}{*}{1999} & \multirow[t]{2}{*}{2000} & \multirow[t]{2}{*}{2001} & \multicolumn{4}{|c|}{$20021 /$} & \multicolumn{4}{|c|}{$20031 /$} \\
\hline & & & & Q1 & Q2 & Q3 & $\overline{\mathrm{Q} 4}$ & & & & \\
\hline Total revenues and grants & 8.9 & 10.3 & 10.4 & 8.8 & 8.6 & 7.9 & 8.0 & 8.2 & 8.1 & 7.8 & 8.0 \\
\hline Total revenues & 9.0 & 10.3 & 10.3 & 8.8 & 8.6 & 7.9 & 8.0 & 8.2 & 8.1 & 7.8 & 8.0 \\
\hline Oil revenues & 0.5 & 1.5 & 0.4 & 0.4 & 0.4 & 0.4 & 0.4 & 0.3 & 0.3 & 0.3 & 0.3 \\
\hline Non-oil revenues & 8.5 & 8.7 & 10.0 & 8.4 & 8.1 & 7.5 & 7.6 & 7.9 & 7.9 & 7.6 & 7.7 \\
\hline Current revenues & 9.0 & 10.3 & 10.3 & 8.7 & 8.5 & 7.8 & 7.9 & 8.1 & 8.0 & 7.7 & 7.8 \\
\hline Capital revenues & 0.0 & 0.0 & 0.1 & 0.1 & 0.1 & 0.1 & 0.1 & 0.1 & 0.1 & 0.1 & 0.1 \\
\hline Tax revenues & 8.5 & 9.9 & 9.9 & 8.5 & 8.3 & 7.7 & 7.8 & 8.1 & 7.9 & 7.6 & 7.7 \\
\hline Income tax & $\ldots$ & $\ldots$ & 4.5 & 2.2 & 2.2 & 2.0 & 2.0 & 2.1 & 2.1 & 2.0 & 2.1 \\
\hline Corporate income tax & $\ldots$ & $\ldots$ & 2.4 & 0.0 & 0.0 & 0.0 & 0.0 & 0.0 & 0.0 & 0.0 & 0.0 \\
\hline Oil revenues & $\ldots$ & $\ldots$ & 0.0 & 0.0 & 0.0 & 0.0 & 0.0 & 0.0 & 0.0 & 0.0 & 0.0 \\
\hline Non-oil revenues & $\ldots$ & $\ldots$ & 2.4 & 0.0 & 0.0 & 0.0 & 0.0 & 0.0 & 0.0 & 0.0 & 0.0 \\
\hline Personal income tax & $\ldots$ & $\ldots$ & 2.1 & 2.2 & 2.2 & 2.0 & 2.0 & 2.1 & 2.1 & 2.0 & 2.1 \\
\hline Social tax & $\ldots$ & $\ldots$ & 3.8 & 3.7 & 3.7 & 3.5 & 3.5 & 3.6 & 3.5 & 3.4 & 3.6 \\
\hline Property taxes & $\ldots$ & $\ldots$ & 1.0 & 1.1 & 1.1 & 0.9 & 0.9 & 1.0 & 0.9 & 0.9 & 0.9 \\
\hline Domestic taxes on goods, works and services & $\ldots$ & $\ldots$ & 0.6 & 1.4 & 1.4 & 1.2 & 1.2 & 1.2 & 1.2 & 1.1 & 1.1 \\
\hline VAT & $\ldots$ & $\ldots$ & 0.3 & 0.3 & 0.3 & 0.2 & 0.3 & 0.2 & 0.2 & 0.2 & 0.2 \\
\hline Excises & $\ldots$ & $\ldots$ & 0.1 & 0.7 & 0.6 & 0.6 & 0.6 & 0.4 & 0.5 & 0.5 & 0.5 \\
\hline Receipts from the use of natural resources & $\ldots$ & $\ldots$ & 0.0 & 0.4 & 0.4 & 0.3 & 0.3 & 0.5 & 0.4 & 0.4 & 0.4 \\
\hline Oil revenues 2/ & $\ldots$ & $\ldots$ & 0.0 & 0.0 & 0.0 & 0.0 & 0.0 & 0.0 & 0.0 & 0.0 & 0.0 \\
\hline Non-oil revenues & $\ldots$ & $\ldots$ & 0.0 & 0.4 & 0.4 & 0.3 & 0.3 & 0.5 & 0.4 & 0.4 & 0.4 \\
\hline Business and professional licences & $\ldots$ & $\ldots$ & 0.1 & 0.1 & 0.1 & 0.1 & 0.1 & 0.1 & 0.0 & 0.1 & 0.1 \\
\hline Taxes on international trade and external transactions & $\ldots$ & $\ldots$ & 0.0 & 0.0 & 0.0 & 0.0 & 0.0 & 0.0 & 0.0 & 0.0 & 0.0 \\
\hline Other taxes & $\ldots$ & $\ldots$ & 0.0 & 0.1 & 0.1 & 0.1 & 0.1 & 0.1 & 0.1 & 0.1 & 0.1 \\
\hline Non-tax revenues & 0.5 & 0.4 & 0.4 & 0.1 & 0.1 & 0.1 & 0.1 & 0.1 & 0.1 & 0.1 & 0.1 \\
\hline Income from capital transactions & 0.0 & 0.0 & 0.1 & 0.1 & 0.1 & 0.1 & 0.1 & 0.1 & 0.1 & 0.1 & 0.1 \\
\hline Grants & -0.1 & 0.0 & 0.0 & 0.0 & 0.0 & 0.0 & 0.0 & 0.0 & 0.0 & 0.0 & 0.0 \\
\hline Total expenditure and net lending and transfers & 8.5 & 9.9 & 10.5 & 9.1 & 8.4 & 7.8 & 8.2 & 6.8 & 7.4 & 7.4 & 8.0 \\
\hline Total expenditures and net lending & 8.2 & 9.4 & 9.4 & $\ldots$ & 9.1 & 8.6 & 8.9 & 8.1 & 9.2 & 9.0 & 9.4 \\
\hline Total expenditures & 8.4 & 9.4 & 9.2 & $\ldots$ & 8.9 & 8.4 & 8.9 & 8.0 & 9.0 & 8.9 & 9.3 \\
\hline Current expenditures & 7.9 & 8.3 & 7.9 & $\ldots$ & 7.2 & 6.6 & 6.9 & 6.9 & 7.1 & 6.7 & 6.8 \\
\hline Expenditures on goods and services & $\ldots$ & $\ldots$ & 6.8 & $\ldots$ & 6.1 & 5.6 & 5.8 & 5.8 & 6.1 & 5.7 & 5.8 \\
\hline Wages & $\ldots$ & $\ldots$ & 2.1 & $\ldots$ & 2.7 & 2.4 & 2.4 & 2.6 & 2.6 & 2.4 & 2.3 \\
\hline Employers' contributions & $\ldots$ & $\ldots$ & 0.4 & $\ldots$ & 0.5 & 0.4 & 0.4 & 0.4 & 0.4 & 0.4 & 0.4 \\
\hline Goods and services & $\ldots$ & $\ldots$ & 4.2 & $\ldots$ & 2.8 & 2.7 & 2.9 & 2.8 & 2.9 & 2.9 & 3.0 \\
\hline Other current expenditures & $\ldots$ & $\ldots$ & 0.1 & $\ldots$ & 0.1 & 0.1 & 0.1 & 0.1 & 0.1 & 0.1 & 0.1 \\
\hline Interest & 0.0 & 0.0 & 0.0 & $\ldots$ & 0.0 & 0.0 & 0.0 & 0.0 & 0.0 & 0.0 & 0.0 \\
\hline Domestic & .. & .. & 0.0 & $\ldots$ & 0.0 & 0.0 & 0.0 & 0.0 & 0.0 & 0.0 & 0.0 \\
\hline Foreign & .. & .. & 0.0 & $\ldots$ & 0.0 & 0.0 & 0.0 & 0.0 & 0.0 & 0.0 & 0.0 \\
\hline Current transfers &.. &.. & 1.1 & $\ldots$ & 1.1 & 1.0 & 1.0 & 1.1 & 1.0 & 0.9 & 0.9 \\
\hline Capital expenditures & 0.5 & 1.1 & 1.2 & $\ldots$ & 1.6 & 1.7 & 2.0 & 1.2 & 1.9 & 2.3 & 2.5 \\
\hline of which: Capital transfers to other levels of government & $\ldots$ & $\cdots$ & $\ldots$ & $\ldots$ & 0.0 & 0.0 & 0.0 & 0.0 & 0.0 & 0.0 & 0.0 \\
\hline Net lending & -0.2 & 0.0 & 0.2 & $\ldots$ & 0.2 & 0.2 & 0.1 & 0.1 & 0.2 & 0.1 & 0.1 \\
\hline Extending of credits; share participation & $\ldots$ & $\ldots$ & 0.4 & $\ldots$ & 0.3 & 0.3 & 0.2 & 0.2 & 0.3 & 0.2 & 0.2 \\
\hline Repayment of credits & $\ldots$ & $\ldots$ & 0.2 & $\ldots$ & 0.1 & 0.1 & 0.2 & 0.1 & 0.0 & 0.1 & 0.0 \\
\hline Net transfers to other levels of government & 0.3 & 0.5 & 1.1 & $\ldots$ & -0.7 & -0.7 & -0.7 & -1.4 & -1.8 & -1.6 & -1.5 \\
\hline Transfers from other levels of government & 1.5 & 1.6 & 1.2 & $\ldots$ & 2.0 & 1.8 & 1.7 & 2.4 & 2.3 & 2.0 & 1.9 \\
\hline Transfers to other levels of government & 1.8 & 2.1 & 2.6 & $\ldots$ & 1.6 & 1.4 & 1.3 & 1.6 & 1.6 & 1.5 & 1.3 \\
\hline Capital transfer from other levels of government & $\ldots$ & $\ldots$ & 0.2 & $\ldots$ & 0.3 & 0.4 & 0.3 & 0.6 & 1.0 & 1.0 & 0.9 \\
\hline Overall budget balance & 0.4 & 0.4 & -0.1 & -0.3 & 0.2 & 0.1 & -0.2 & 1.5 & 0.7 & 0.4 & 0.0 \\
\hline Statistical discrepancy & 0.0 & 0.4 & -0.1 & $\ldots$ & 0.3 & 0.1 & -0.2 & 1.8 & 1.0 & 0.5 & 0.0 \\
\hline Financing & -0.4 & 0.0 & 0.0 & 0.3 & 0.1 & 0.0 & 0.0 & 0.3 & 0.2 & 0.1 & 0.0 \\
\hline Domestic financing (net) & -0.6 & 0.0 & 0.0 & 0.3 & 0.0 & 0.0 & 0.0 & 0.3 & 0.2 & 0.1 & 0.0 \\
\hline Banking system & $\ldots$ & $\ldots$ & 0.0 & 0.3 & 0.0 & 0.0 & 0.0 & 0.3 & 0.2 & 0.1 & 0.0 \\
\hline National Bank of Kazakhstan & $\ldots$ & $\ldots$ & 0.0 & 0.0 & 0.0 & 0.0 & 0.0 & 0.0 & 0.0 & 0.0 & 0.0 \\
\hline Commercial banks & $\ldots$ & $\ldots$ & 0.0 & 0.3 & 0.0 & 0.0 & 0.0 & 0.3 & 0.2 & 0.1 & 0.0 \\
\hline Non-bank financing & & $\ldots$ & 0.0 & 0.0 & 0.0 & 0.0 & 0.0 & 0.0 & 0.0 & 0.0 & 0.0 \\
\hline Foreign financing, net & 0.0 & 0.0 & 0.0 & 0.0 & 0.0 & 0.0 & 0.0 & 0.0 & 0.0 & 0.0 & 0.0 \\
\hline Disbursements & .. & .. & $\ldots$ & $\ldots$ & $\ldots$ & $\ldots$ & $\ldots$ & $\ldots$ & $\ldots$ & $\ldots$ & $\ldots$ \\
\hline Amortization & & & $\ldots$ & & & & & $\ldots$ & $\ldots$ & & \\
\hline Privatization receipts & 0.1 & 0.0 & 0.0 & 0.0 & 0.0 & 0.0 & 0.0 & 0.0 & 0.0 & 0.0 & 0.0 \\
\hline National Fund of the Republic of Kazakhstan (NFRK) & $\ldots$ & $\cdots$ & $\cdots$ & $\cdots$ & $\cdots$ & $\cdots$ & $\cdots$ & $\ldots$ & $\cdots$ & $\ldots$ & $\cdots$ \\
\hline \multicolumn{12}{|l|}{ Memorandum items: } \\
\hline Nominal GDP (in billions of tenge) & 2,016 & 2,600 & 3,251 & 746 & 1,666 & 2,754 & 3,776 & 933 & 2,041 & 3,271 & 4,450 \\
\hline
\end{tabular}

Source: Kazakhstani authorities, Fund staff estimates.

$1 /$ Cumulative since the end of the previous year.

2/ Oil royalties, bonuses, and production sharing agreements. 
Table 21. Kazakhstan: General Government Expenditure by Functional Classification, 1999-2003

\begin{tabular}{|c|c|c|c|c|c|c|c|c|c|c|c|}
\hline & \multirow[t]{2}{*}{1999} & \multirow[t]{2}{*}{2000} & \multirow[t]{2}{*}{2001} & \multicolumn{4}{|c|}{$20021 /$} & \multicolumn{4}{|c|}{$20031 /$} \\
\hline & & & & Q1 & Q2 & Q3 & Q4 & Q1 & Q2 & Q3 & Q4 \\
\hline & \multicolumn{11}{|c|}{ (In billions of tenge) } \\
\hline Expenditure and net lending & 458.3 & 584.2 & 746.2 & 141.0 & 350.2 & 548.7 & 794.0 & 186.0 & 453.0 & 745.5 & $1,036.0$ \\
\hline Expenditure & 440.3 & 567.0 & 724.8 & 138.4 & 339.4 & 531.4 & 775.6 & 180.8 & 439.3 & 700.8 & 985.7 \\
\hline General government services & 28.9 & 34.8 & 50.8 & 8.5 & 19.0 & 30.0 & 45.1 & 12.5 & 26.3 & 45.3 & 64.0 \\
\hline Defense & 17.2 & 20.5 & 32.5 & 5.1 & 13.5 & 22.4 & 37.7 & 7.1 & 18.4 & 31.5 & 47.5 \\
\hline Public order and security & 32.5 & 46.9 & 64.3 & 13.5 & 32.5 & 52.4 & 77.7 & 18.5 & 43.3 & 67.0 & 91.6 \\
\hline Education & 78.5 & 84.6 & 106.4 & 23.8 & 58.9 & 85.9 & 121.1 & 32.1 & 73.5 & 108.7 & 148.9 \\
\hline Health & 44.8 & 54.2 & 62.3 & 12.7 & 31.0 & 50.3 & 71.1 & 16.8 & 39.4 & 64.5 & 89.8 \\
\hline Social insurance and social security & 159.1 & 171.0 & 186.7 & 46.1 & 98.4 & 149.2 & 201.3 & 52.2 & 108.2 & 171.4 & 239.2 \\
\hline Housing and public utilities & 6.0 & 22.0 & 30.5 & 3.9 & 9.0 & 15.4 & 24.5 & 4.2 & 13.0 & 22.2 & 34.0 \\
\hline Culture, sports, and information space & 12.2 & 17.5 & 18.1 & 4.0 & 10.6 & 15.9 & 22.8 & 6.8 & 15.9 & 24.8 & 33.8 \\
\hline Fuel and energy complex, and subsoil use & 0.0 & 0.0 & 5.5 & 0.6 & 2.3 & 5.2 & 6.8 & 1.9 & 5.6 & 7.2 & 8.5 \\
\hline Agriculture, water industry, forestry, and fishery & 6.9 & 11.4 & 23.2 & 1.2 & 10.3 & 18.2 & 27.7 & 3.4 & 18.1 & 34.6 & 45.5 \\
\hline Industry and construction & 2.9 & 7.2 & 4.8 & 0.1 & 0.7 & 2.2 & 4.5 & 0.3 & 1.1 & 2.1 & 3.9 \\
\hline Transportation and communications & 12.9 & 33.3 & 44.2 & 3.3 & 12.3 & 28.5 & 49.6 & 13.6 & 38.9 & 64.2 & 81.2 \\
\hline Other expenditure & 19.1 & 28.1 & 58.0 & 10.3 & 22.0 & 31.2 & 46.6 & 7.7 & 19.6 & 35.4 & 62.5 \\
\hline Debt servicing & 19.4 & 35.4 & 37.7 & 5.1 & 19.0 & 24.5 & 39.0 & 3.8 & 18.0 & 21.8 & 35.4 \\
\hline Net lending & 18.0 & 17.2 & 21.4 & 2.6 & 10.8 & 17.3 & 18.4 & 5.2 & 13.7 & 44.8 & 50.7 \\
\hline Lending & 21.0 & 25.7 & 34.1 & 5.2 & 15.6 & 23.3 & 31.7 & 7.1 & 20.1 & 54.0 & 68.3 \\
\hline \multirow[t]{2}{*}{ Repayments } & -3.0 & -8.5 & -12.7 & -2.6 & -4.8 & -6.0 & -13.3 & 1.8 & 6.4 & 9.2 & 17.7 \\
\hline & \multicolumn{11}{|c|}{ (In percent of GDP) } \\
\hline Expenditure and net lending & 22.7 & 22.5 & 23.0 & 18.3 & 21.0 & 19.9 & 21.2 & 19.9 & 22.2 & 22.8 & 23.3 \\
\hline Expenditure & 21.8 & 21.8 & 22.3 & 17.9 & 20.3 & 19.3 & 20.7 & 19.4 & 21.5 & 21.4 & 22.2 \\
\hline General government services & 1.4 & 1.3 & 1.6 & 1.1 & 1.1 & 1.1 & 1.2 & 1.3 & 1.3 & 1.4 & 1.4 \\
\hline Defense & 0.9 & 0.8 & 1.0 & 0.7 & 0.8 & 0.8 & 1.0 & 0.8 & 0.9 & 1.0 & 1.1 \\
\hline Public order and security & 1.6 & 1.8 & 2.0 & 1.8 & 1.9 & 1.9 & 2.1 & 2.0 & 2.1 & 2.0 & 2.1 \\
\hline Education & 3.9 & 3.3 & 3.3 & 3.1 & 3.5 & 3.1 & 3.2 & 3.4 & 3.6 & 3.3 & 3.3 \\
\hline Health & 2.2 & 2.1 & 1.9 & 1.6 & 1.9 & 1.8 & 1.9 & 1.8 & 1.9 & 2.0 & 2.0 \\
\hline Social insurance and social security & 7.9 & 6.6 & 5.7 & 6.0 & 5.9 & 5.4 & 5.4 & 5.6 & 5.3 & 5.2 & 5.4 \\
\hline Housing and public utilities & 0.3 & 0.8 & 0.9 & 0.5 & 0.5 & 0.6 & 0.7 & 0.5 & 0.6 & 0.7 & 0.8 \\
\hline Culture, sports, and information space & 0.6 & 0.7 & 0.6 & 0.5 & 0.6 & 0.6 & 0.6 & 0.7 & 0.8 & 0.8 & 0.8 \\
\hline Fuel and energy complex, and subsoil use & 0.0 & 0.0 & 0.2 & 0.1 & 0.1 & 0.2 & 0.2 & 0.2 & 0.3 & 0.2 & 0.2 \\
\hline Agriculture, water industry, forestry, and fishery & 0.3 & 0.4 & 0.7 & 0.2 & 0.6 & 0.7 & 0.7 & 0.4 & 0.9 & 1.1 & 1.0 \\
\hline Industry and construction & 0.1 & 0.3 & 0.1 & 0.0 & 0.0 & 0.1 & 0.1 & 0.0 & 0.1 & 0.1 & 0.1 \\
\hline Transport and communications & 0.6 & 1.3 & 1.4 & 0.4 & 0.7 & 1.0 & 1.3 & 1.5 & 1.9 & 2.0 & 1.8 \\
\hline Other expenditure & 0.9 & 1.1 & 1.8 & 1.3 & 1.3 & 1.1 & 1.2 & 0.8 & 1.0 & 1.1 & 1.4 \\
\hline Debt servicing & 1.0 & 1.4 & 1.2 & 0.7 & 1.1 & 0.9 & 1.0 & 0.4 & 0.9 & 0.7 & 0.8 \\
\hline Net lending & 0.9 & 0.7 & 0.7 & 0.3 & 0.6 & 0.6 & 0.5 & 0.6 & 0.7 & 1.4 & 1.1 \\
\hline Lending & 1.0 & 1.0 & 1.0 & 0.7 & 0.9 & 0.8 & 0.8 & 0.8 & 1.0 & 1.6 & 1.5 \\
\hline \multirow[t]{2}{*}{ Repayments } & -0.1 & -0.3 & -0.4 & -0.3 & -0.3 & -0.2 & -0.4 & 0.2 & 0.3 & 0.3 & 0.4 \\
\hline & \multicolumn{11}{|c|}{ (In percent of total) } \\
\hline Expenditure and net lending & 100.0 & 100.0 & 100.0 & 100.0 & 100.0 & 100.0 & 100.0 & 100.0 & 100.0 & 100.0 & 100.0 \\
\hline Expenditure & 96.1 & 97.1 & 97.1 & 98.2 & 96.9 & 96.9 & 97.7 & 97.2 & 97.0 & 94.0 & 95.1 \\
\hline General government services & 6.3 & 6.0 & 6.8 & 6.0 & 5.4 & 5.5 & 5.7 & 6.7 & 5.8 & 6.1 & 6.2 \\
\hline Defense & 3.8 & 3.5 & 4.4 & 3.6 & 3.9 & 4.1 & 4.7 & 3.8 & 4.1 & 4.2 & 4.6 \\
\hline Public order and security & 7.1 & 8.0 & 8.6 & 9.6 & 9.3 & 9.6 & 9.8 & 9.9 & 9.6 & 9.0 & 8.8 \\
\hline Education & 17.1 & 14.5 & 14.3 & 16.9 & 16.8 & 15.7 & 15.3 & 17.2 & 16.2 & 14.6 & 14.4 \\
\hline Health & 9.8 & 9.3 & 8.4 & 9.0 & 8.8 & 9.2 & 9.0 & 9.0 & 8.7 & 8.7 & 8.7 \\
\hline Social insurance and social security & 34.7 & 29.3 & 25.0 & 32.7 & 28.1 & 27.2 & 25.4 & 28.0 & 23.9 & 23.0 & 23.1 \\
\hline Housing and public utilities & 1.3 & 3.8 & 4.1 & 2.8 & 2.6 & 2.8 & 3.1 & 2.3 & 2.9 & 3.0 & 3.3 \\
\hline Culture, sports, and information space & 2.7 & 3.0 & 2.4 & 2.8 & 3.0 & 2.9 & 2.9 & 3.6 & 3.5 & 3.3 & 3.3 \\
\hline Fuel and energy complex, and subsoil use & 0.0 & 0.0 & 0.7 & 0.5 & 0.7 & 1.0 & 0.9 & 1.0 & 1.2 & 1.0 & 0.8 \\
\hline Agriculture, water industry, forestry, and fishery & 1.5 & 2.0 & 3.1 & 0.9 & 2.9 & 3.3 & 3.5 & 1.8 & 4.0 & 4.6 & 4.4 \\
\hline Industry and construction & 0.6 & 1.2 & 0.6 & 0.1 & 0.2 & 0.4 & 0.6 & 0.2 & 0.3 & 0.3 & 0.4 \\
\hline Transport and communications & 2.8 & 5.7 & 5.9 & 2.4 & 3.5 & 5.2 & 6.2 & 7.3 & 8.6 & 8.6 & 7.8 \\
\hline Other expenditure & 4.2 & 4.8 & 7.8 & 7.3 & 6.3 & 5.7 & 5.9 & 4.2 & 4.3 & 4.8 & 6.0 \\
\hline Debt servicing & 4.2 & 6.1 & 5.0 & 3.6 & 5.4 & 4.5 & 4.9 & 2.0 & 4.0 & 2.9 & 3.4 \\
\hline Net lending & 3.9 & 2.9 & 2.9 & 1.8 & 3.1 & 3.1 & 2.3 & 2.8 & 3.0 & 6.0 & 4.9 \\
\hline Lending & 4.6 & 4.4 & 4.6 & 3.7 & 4.4 & 4.2 & 4.0 & 3.8 & 4.4 & 7.2 & 6.6 \\
\hline Repayments & -0.7 & -1.5 & -1.7 & -1.9 & -1.4 & -1.1 & -1.7 & 1.0 & 1.4 & 1.2 & 1.7 \\
\hline \multicolumn{12}{|l|}{ Memorandum items: } \\
\hline Nominal GDP (in billions of tenge) & 2,016 & 2,600 & 3,251 & 746 & 1,666 & 2,754 & 3,776 & 933 & 2,041 & 3,271 & 4,450 \\
\hline
\end{tabular}

Source: Kazakhstani authorities.

$1 /$ Cumulative since the end of the previous year. 
Table 22. Kazakhstan: Balance of Payments, 1999-2003

(In millions of U.S.dollars, unless otherwise indicated)

\begin{tabular}{|c|c|c|c|c|c|}
\hline & 1999 & 2000 & 2001 & 2002 & $\begin{array}{c}2003 \\
\text { Preliminary }\end{array}$ \\
\hline Current account & -9 & 768 & -896 & -862 & -69 \\
\hline Trade balance & 478 & 2,620 & 1,516 & 2,301 & 4,088 \\
\hline Exports (f.o.b.) & 6,123 & 9,468 & 9,124 & 10,027 & 13,233 \\
\hline Oil and gas condensate & 2,174 & 4,429 & 4,463 & 5,037 & 7,023 \\
\hline Non-oil exports & 3,948 & 5,039 & 4,661 & 4,990 & 6,209 \\
\hline Imports (f.o.b.) & $-5,645$ & $-6,848$ & $-7,607$ & $-7,726$ & $-9,144$ \\
\hline Services and income balance & -644 & $-2,101$ & $-2,644$ & $-3,276$ & $-3,992$ \\
\hline Services, net & -171 & -872 & $-1,518$ & $-2,153$ & $-2,251$ \\
\hline Income, net & -472 & $-1,229$ & $-1,126$ & $-1,123$ & $-1,741$ \\
\hline of which: Income to direct investors & -274 & $-1,046$ & $-1,051$ & $-1,007$ & $-1,424$ \\
\hline Current transfers & 157 & 249 & 232 & 114 & -165 \\
\hline Capital and financial account & 903 & 811 & 2,207 & 1,241 & 2,700 \\
\hline Capital transfers, net & -234 & -291 & -194 & -120 & -29 \\
\hline Foreign direct investment & 1583 & 1,278 & 3,071 & 2,163 & 2,188 \\
\hline Amortization of intra-company liabilities & -212 & $-1,411$ & $-1,792$ & $-1,518$ & $-2,261$ \\
\hline Portfolio investment, net & -46 & -55 & $-1,317$ & -927 & $-1,845$ \\
\hline of which: National Fund 1/ & $\ldots$ & $\ldots$ & $-1,270$ & -327 & -964 \\
\hline Public sector Eurobonds, net & 34 & 11 & -103 & -449 & 54 \\
\hline Other investment & -400 & -121 & 648 & 125 & 2,386 \\
\hline Medium- and long-term loans and credits, net & 192 & 243 & 385 & 725 & 2,112 \\
\hline Short-term and other capital, net & -592 & -364 & 263 & -601 & 274 \\
\hline Errors and omissions & -641 & -994 & -926 & 156 & $-1,098$ \\
\hline Overall balance & 254 & 585 & 385 & 535 & 1,534 \\
\hline Financing & -254 & -585 & -385 & -535 & $-1,534$ \\
\hline Net international reserves of the NBK (increase -) & -254 & -585 & -385 & -535 & $-1,534$ \\
\hline \multicolumn{6}{|l|}{ Memorandum items } \\
\hline Current account (in percent of annual GDP) & -0.1 & 4.2 & -4.0 & -3.5 & -0.2 \\
\hline Exports in percent of GDP & 36.1 & 51.8 & 41.2 & 40.8 & 44.5 \\
\hline of which: Oil exports & 12.8 & 24.2 & 20.2 & 20.5 & 23.6 \\
\hline Imports in percent of GDP & 33.3 & 37.4 & 34.4 & 31.4 & 30.8 \\
\hline \multicolumn{6}{|l|}{ Annual growth rate (in percent) } \\
\hline Exports & 4.3 & 54.6 & -3.6 & 9.9 & 32.0 \\
\hline Non-oil exports & -6.4 & 27.6 & -7.5 & 7.1 & 24.4 \\
\hline Imports & -15.4 & 21.3 & 11.1 & 1.6 & 18.4 \\
\hline Exports of crude oil and gas condensate (in millions of tons) & 23.7 & 27.7 & 32.4 & 39.3 & 44.3 \\
\hline NBK gross international reserves & 2,003 & 2,096 & 2,508 & 3,136 & 4,961 \\
\hline In months of imports of goods and non-factor services & 3.6 & 2.8 & 2.9 & 3.3 & 4.5 \\
\hline National Fund (incl. interest), end of period stock & $\ldots$ & $\ldots$ & 1,270 & 1,917 & 3,603 \\
\hline External debt in percent of GDP & 71.3 & 69.3 & 68.5 & 74.1 & 76.9 \\
\hline Excluding intra-company loans & 35.0 & 31.4 & 28.4 & 30.5 & 36.4 \\
\hline Public external debt service in percent of exports of gnfs & 11.8 & 8.5 & 4.8 & 7.9 & 3.2 \\
\hline World oil price (U.S. dollars per barrel) & 18.0 & 28.2 & 24.3 & 24.9 & 28.9 \\
\hline
\end{tabular}

Sources: Kazakhstani authorities; and Fund staff estimates.

1/ For 2001 and 2002 the numbers also reflect the effect of market valuation, in addition to transactions. The start-up assets in 2001 included $\$ 660$ million from the sale of a 5 percent government share in TCO. The remaining balance was from oil revenue. 


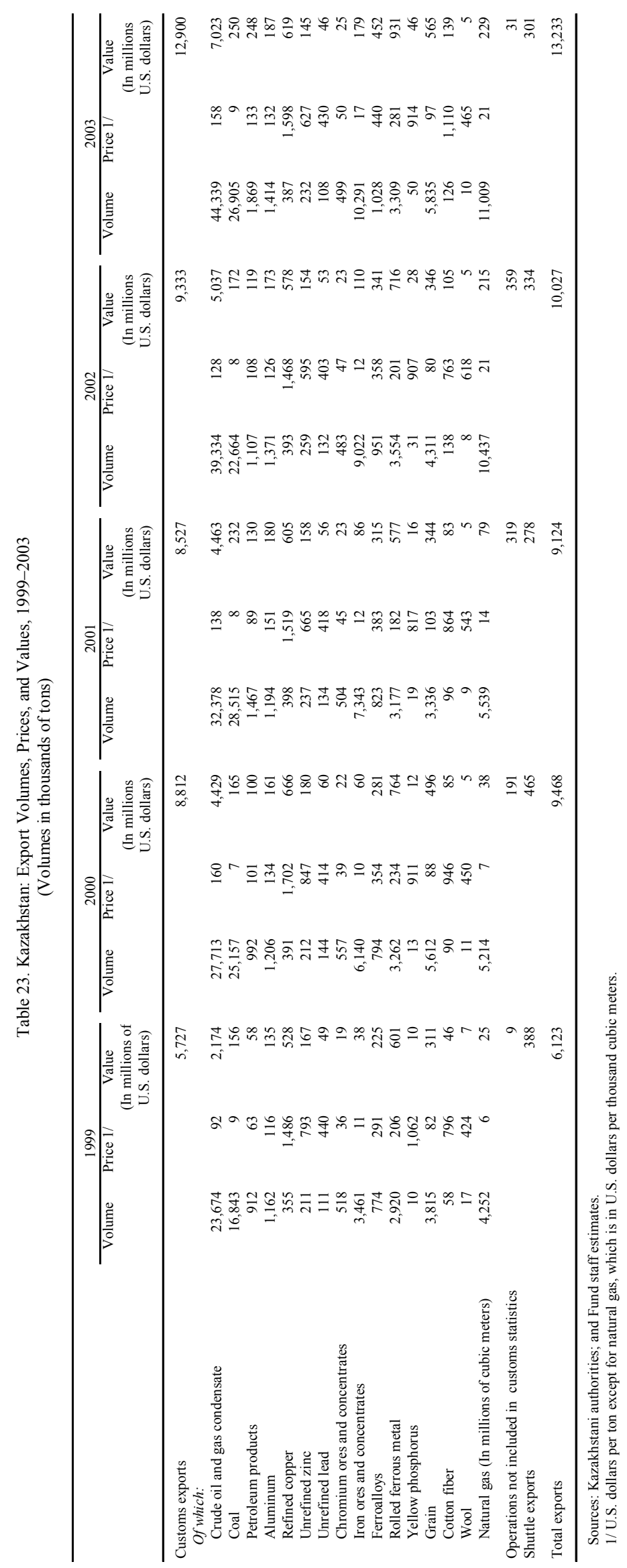




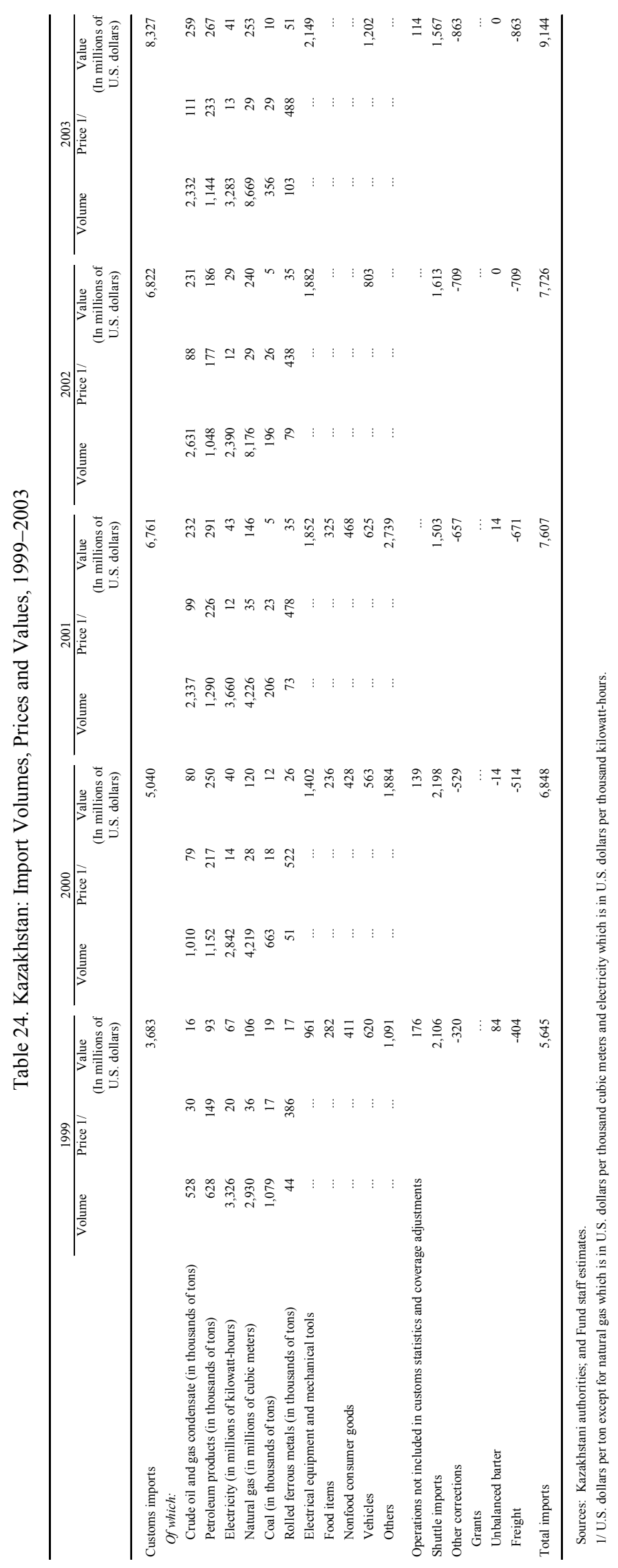


Table 25. Kazakhstan: Geographical Distribution of Exports, 1999-2003 (In percent of total)

\begin{tabular}{|c|c|c|c|c|c|}
\hline & 1999 & 2000 & 2001 & 2002 & 2003 \\
\hline BRO Countries & 29.4 & 27.6 & 31.4 & 23.4 & 24.4 \\
\hline Armenia & 0.1 & 0.0 & 0.0 & 0.0 & 0.8 \\
\hline Azerbaijan & 0.5 & 0.5 & 0.8 & 1.2 & 0.9 \\
\hline Belarus & 0.2 & 0.2 & 0.1 & 0.1 & 0.1 \\
\hline Estonia & 1.9 & 0.1 & 0.1 & 0.1 & 0.1 \\
\hline Georgia & 0.1 & 0.1 & 0.0 & 0.1 & 0.1 \\
\hline Kyrgyz Republic & 1.0 & 0.7 & 1.0 & 1.1 & 1.2 \\
\hline Latvia & 0.4 & 0.8 & 0.5 & 0.6 & 0.6 \\
\hline Lithuania & 1.5 & 0.2 & 0.1 & 0.0 & 0.0 \\
\hline Moldova & 0.0 & 0.0 & 0.0 & 0.0 & 0.3 \\
\hline Russia & 19.5 & 19.9 & 20.4 & 15.5 & 15.2 \\
\hline Tajikistan & 0.7 & 0.6 & 0.7 & 0.5 & 0.6 \\
\hline Turkmenistan & 0.2 & 0.1 & 0.2 & 0.2 & 0.3 \\
\hline Ukraine & 2.2 & 2.9 & 5.7 & 3.0 & 3.2 \\
\hline Uzbekistan & 1.1 & 1.5 & 1.7 & 1.0 & 1.0 \\
\hline Non-BRO Countries & 70.6 & 72.4 & 68.6 & 76.6 & 75.6 \\
\hline Austria & 0.0 & 0.0 & 0.0 & 0.0 & 0.0 \\
\hline Afghanistan & 0.2 & 0.7 & 0.2 & 0.3 & 0.4 \\
\hline Belgium & 0.6 & 0.1 & 0.0 & 0.1 & 0.1 \\
\hline China & 8.0 & 7.6 & 7.6 & 10.6 & 12.8 \\
\hline Czech Republic & 0.1 & 0.1 & 0.0 & 0.2 & 0.2 \\
\hline Finland & 0.7 & 0.8 & 0.6 & 0.5 & 0.9 \\
\hline Greece & 0.0 & 0.0 & 0.1 & 0.3 & 0.1 \\
\hline Germany & 6.0 & 6.3 & 5.8 & 2.3 & 1.1 \\
\hline Hungary & 0.1 & 0.0 & 0.1 & 0.1 & 0.2 \\
\hline Italy & 7.3 & 10.4 & 11.1 & 9.4 & 7.9 \\
\hline Japan & 0.4 & 0.1 & 0.2 & 0.2 & 0.1 \\
\hline Netherlands & 2.8 & 2.6 & 1.7 & 1.3 & 1.4 \\
\hline Oman & 0.0 & 0.0 & 0.0 & 0.0 & 0.0 \\
\hline Poland & 1.6 & 0.6 & 1.9 & 3.3 & 1.6 \\
\hline South Korea & 0.6 & 0.4 & 0.5 & 0.5 & 0.4 \\
\hline Switzerland & 5.6 & 5.1 & 4.7 & 8.2 & 13.0 \\
\hline Sweden & 0.4 & 0.4 & 0.1 & 0.1 & 0.0 \\
\hline Thailand & 0.9 & 0.2 & 0.0 & 0.2 & 0.1 \\
\hline Turkey & 0.6 & 0.7 & 0.9 & 1.0 & 0.8 \\
\hline United Kingdom & 3.2 & 2.6 & 3.4 & 1.4 & 1.1 \\
\hline United States & 1.4 & 2.4 & 1.8 & 1.2 & 0.8 \\
\hline Yugoslavia & 0.0 & 0.0 & 0.0 & 0.0 & 0.0 \\
\hline Other countries & 30.1 & 31.3 & 27.9 & 35.4 & 32.6 \\
\hline
\end{tabular}

Source: Kazakhstani authorities. 
Table 26. Kazakhstan: Geographical Distribution of Imports, 1999-2003

(In percent of total)

\begin{tabular}{|c|c|c|c|c|c|}
\hline & 1999 & 2000 & 2001 & 2002 & 2003 \\
\hline BRO Countries & 44.2 & 54.6 & 51.6 & 46.5 & 47.6 \\
\hline Armenia & 0.0 & 0.0 & 0.0 & 0.0 & 0.0 \\
\hline Azerbaijan & 0.1 & 0.2 & 0.2 & 0.2 & 0.2 \\
\hline Belarus & 1.0 & 0.8 & 0.7 & 0.8 & 1.1 \\
\hline Estonia & 0.0 & 0.0 & 0.0 & 0.1 & 0.1 \\
\hline Georgia & 0.0 & 0.1 & 0.1 & 0.1 & 0.1 \\
\hline Kyrgyz Republic & 0.9 & 0.6 & 0.5 & 0.5 & 0.6 \\
\hline Latvia & 0.1 & 0.1 & 0.1 & 0.2 & 0.2 \\
\hline Lithuania & 0.2 & 0.2 & 0.1 & 0.1 & 0.2 \\
\hline Moldova & 0.1 & 0.1 & 0.1 & 0.1 & 0.1 \\
\hline Russia & 37.0 & 48.4 & 44.9 & 38.7 & 39.3 \\
\hline Tajikistan & 0.1 & 0.1 & 0.0 & 0.0 & 0.1 \\
\hline Turkmenistan & 0.5 & 0.9 & 1.2 & 1.1 & 0.6 \\
\hline Ukraine & 1.6 & 1.6 & 2.4 & 3.3 & 3.9 \\
\hline Uzbekistan & 2.5 & 1.4 & 1.3 & 1.3 & 1.1 \\
\hline Non-BRO Countries & 55.9 & 45.5 & 48.5 & 53.5 & 52.4 \\
\hline Austria & 0.5 & 0.4 & 0.5 & 0.7 & 1.0 \\
\hline Canada & 0.4 & 0.5 & 0.6 & 0.7 & 0.7 \\
\hline China & 2.2 & 3.0 & 2.7 & 4.8 & 6.0 \\
\hline Cuba & 0.5 & 0.6 & 0.1 & 0.3 & 0.2 \\
\hline Czech Republic & 0.7 & 0.7 & 0.7 & 0.8 & 0.5 \\
\hline Finland & 1.2 & 1.1 & 1.1 & 1.1 & 1.2 \\
\hline Germany & 7.7 & 6.7 & 7.6 & 8.9 & 8.9 \\
\hline Hungary & 1.0 & 0.5 & 0.5 & 0.6 & 0.5 \\
\hline India & 0.8 & 0.9 & 0.7 & 0.8 & 0.9 \\
\hline Italy & 2.9 & 3.1 & 4.2 & 3.3 & 2.9 \\
\hline Japan & 3.2 & 2.1 & 2.2 & 2.5 & 2.4 \\
\hline Poland & 1.7 & 1.2 & 1.0 & 1.1 & 1.4 \\
\hline Switzerland & 1.1 & 1.1 & 1.0 & 0.9 & 0.8 \\
\hline Sweden & 0.7 & 0.5 & 0.7 & 0.8 & 1.5 \\
\hline United Kingdom & 6.2 & 4.4 & 3.9 & 3.9 & 3.0 \\
\hline United States & 9.4 & 5.5 & 5.4 & 7.0 & 5.6 \\
\hline Yugoslavia & 0.0 & 0.0 & 0.0 & 0.0 & 0.0 \\
\hline Other countries & 15.7 & 13.2 & 15.5 & 15.3 & 14.9 \\
\hline
\end{tabular}

Source: Kazakhstani authorities. 
Table 27. Kazakhstan: Geographical Distribution of Exports of Energy Products to the Baltics, Russia, and Other States of the Former Soviet Union, 1999-2003

\begin{tabular}{|c|c|c|c|c|c|}
\hline & 1999 & 2000 & 2001 & 2002 & 2003 \\
\hline & \multicolumn{5}{|c|}{ (In thousands of metric tons) } \\
\hline $\begin{array}{l}\text { Oil and gas condensate } \\
\text { of which: }\end{array}$ & $7,751.7$ & $7,943.5$ & $9,076.9$ & $8,828.8$ & $7,229.6$ \\
\hline Russia & $5,385.5$ & $6,178.0$ & $5,346.4$ & $6,349.1$ & $5,714.8$ \\
\hline Ukraine & $1,661.5$ & $1,737.9$ & $3,730.5$ & $2,475.7$ & $1,287.5$ \\
\hline \multirow[t]{2}{*}{ Uzbekistan } & $\cdots$ & $\ldots$ & $\cdots$ & $\cdots$ & 210.6 \\
\hline & \multicolumn{5}{|c|}{ (In millions of cubic meters) } \\
\hline $\begin{array}{l}\text { Natural gas } \\
\text { of which: }\end{array}$ & $3,776.2$ & $4,934.3$ & $4,784.6$ & $9,378.6$ & $9,382.0$ \\
\hline Azerbaijan & $\ldots$ & $\ldots$ & $\ldots$ & $1,910.8$ & 718.5 \\
\hline Armenia & $\ldots$ & $\ldots$ & $\ldots$ & 50.6 & 197.5 \\
\hline Georgia & 127.4 & 271.7 & 10.0 & 73.1 & 115.2 \\
\hline Kyrgyz Republic & $\ldots$ & $\ldots$ & $\ldots$ & 22.4 & 31.0 \\
\hline Moldova & $\ldots$ & $\ldots$ & $\ldots$ & 35.0 & 782.5 \\
\hline Russia & $3,648.8$ & $4,662.6$ & $4,774.6$ & $7,167.1$ & $7,057.3$ \\
\hline Ukraine & $\ldots$ & $\ldots$ & $\ldots$ & 80.3 & 480.0 \\
\hline \multirow[t]{2}{*}{ Uzbekistan } & $\ldots$ & $\ldots$ & $\ldots$ & 39.3 & $\ldots$ \\
\hline & \multicolumn{5}{|c|}{ (In thousands of metric tons) } \\
\hline $\begin{array}{l}\text { Gasoline } \\
\text { of which: }\end{array}$ & 39.8 & 59.2 & 91.1 & 102.4 & 143.6 \\
\hline Kyrgyz Republic & 36.3 & 35.0 & 65.9 & 84.0 & 104.6 \\
\hline Russia & 0.0 & 19.8 & $\ldots$ & $\ldots$ & $\ldots$ \\
\hline Tajikistan & 2.0 & 4.4 & 24.2 & 15.9 & 38.1 \\
\hline Uzbekistan & 1.5 & 0.0 & 1.0 & 1.3 & $\ldots$ \\
\hline $\begin{array}{l}\text { Diesel fuel } \\
\text { of which: }\end{array}$ & 50.8 & 19.9 & 189.2 & 55.3 & 99.9 \\
\hline Kyrgyz Republic & 41.1 & 19.7 & 30.2 & 43.4 & 51.0 \\
\hline Russia & 8.6 & 0.2 & 158.7 & $\ldots$ & 1.7 \\
\hline Tajikistan & 1.1 & $\ldots$ & 0.2 & 4.6 & 45.8 \\
\hline Uzbekistan & $\ldots$ & $\ldots$ & 0.1 & 1.3 & 1.4 \\
\hline $\begin{array}{l}\text { Heavy furnace fuel } \\
\text { of which: }\end{array}$ & 90.2 & 46.4 & 153.8 & 8.4 & 59.4 \\
\hline Georgia & $\ldots$ & $\ldots$ & $\ldots$ & $\ldots$ & 4.2 \\
\hline Kyrgyz Republic & 32.7 & 0.0 & 11.2 & 8.4 & 35.0 \\
\hline Lithuania & 56.9 & 20.4 & $\ldots$ & $\ldots$ & $\ldots$ \\
\hline Russia & 0.6 & 25.0 & 126.5 & $\ldots$ & 0.4 \\
\hline Tajikistan & $\ldots$ & $\ldots$ & 3.4 & $\ldots$ & 14.8 \\
\hline Ukraine & $\ldots$ & 1.0 & 12.9 & $\ldots$ & 5.0 \\
\hline $\begin{array}{l}\text { Coking coal } \\
\text { of which: }\end{array}$ & 2.5 & 91.4 & 937.6 & 252.2 & $1,165.7$ \\
\hline Russia & 2.5 & 91.4 & 935.5 & 240.5 & $1,004.8$ \\
\hline Uzbekistan & 0.0 & 0.0 & $\ldots$ & 0.8 & $\ldots$ \\
\hline Ukraine & 0.0 & 0.0 & 2.1 & 10.9 & 160.9 \\
\hline
\end{tabular}

Source: Kazakhstani authorities. 
Table 28. Kazakhstan: Breakdown of Foreign Direct Investment by Country of Origin, 1999-2003 (In percent of total)

\begin{tabular}{|c|c|c|c|c|c|}
\hline Country & 1999 & 2000 & 2001 & 2002 & 2003 \\
\hline Canada & 0.5 & 5.7 & 10.8 & 4.0 & 0.2 \\
\hline China & 2.7 & 3.2 & 4.7 & 1.6 & 5.4 \\
\hline Germany & 0.8 & 2.5 & 1.1 & 0.9 & 1.4 \\
\hline Iceland & 0.0 & 0.0 & 0.0 & 0.0 & 0.0 \\
\hline Indonesia & 0.0 & 2.4 & 1.0 & 0.0 & 0.0 \\
\hline South Korea & 1.6 & 2.0 & 1.5 & 1.1 & 1.8 \\
\hline Switzerland & 1.3 & 0.7 & 7.9 & 12.7 & 13.7 \\
\hline Turkey & 1.7 & 1.1 & 1.3 & 1.7 & 2.3 \\
\hline United Kingdom & 8.6 & 17.3 & 13.2 & 15.2 & 12.9 \\
\hline United States & 48.9 & 34.2 & 32.1 & 24.6 & 23.6 \\
\hline Others & 33.9 & 30.7 & 26.5 & 38.2 & 38.7 \\
\hline Total & 100.0 & 100.0 & 100.0 & 100.0 & 100.0 \\
\hline
\end{tabular}

Source: National Bank of Kazakhstan. 
Table 29. Kazakhstan: Breakdown of Foreign Direct Investment by Industry, 1999-2003

(In percent of total)

\begin{tabular}{|c|c|c|c|c|c|}
\hline Sector & 1999 & 2000 & 2001 & 2002 & 2003 \\
\hline Agriculture, Hunting, and Related Services & 0.0 & 0.1 & 0.1 & 0.1 & 0.0 \\
\hline $\begin{array}{l}\text { Mining and Quarrying } \\
\text { of which: }\end{array}$ & 75.7 & 73.2 & 67.8 & 51.7 & 47.6 \\
\hline Coal and lignite; extraction of peat & 0.1 & 0.6 & 0.0 & 0.2 & 0.1 \\
\hline Crude petroleum, natural gas, and related service activities & 74.1 & 72.0 & 67.1 & 50.4 & 46.0 \\
\hline Mining of uranium, metal, and thorium ores & 1.6 & 0.3 & 0.5 & 1.0 & 1.0 \\
\hline $\begin{array}{l}\text { Manufacturing } \\
\text { of which: }\end{array}$ & 9.1 & 8.9 & 14.1 & 20.3 & 21.6 \\
\hline Farm products & 4.2 & 1.5 & 2.0 & 1.6 & 0.8 \\
\hline Coke, refined petroleum products, and nuclear fuel & 0.9 & 1.1 & 1.4 & 2.6 & 4.3 \\
\hline Chemicals and chemical products & 0.3 & 0.3 & 0.2 & 0.5 & 0.1 \\
\hline Basic metals and fabricated metal products, except machinery and equipment & 1.9 & 3.6 & 8.5 & 14.0 & 13.6 \\
\hline $\begin{array}{l}\text { Office, accounting, and communication equipment; medical, precision, and } \\
\text { instruments; watches and clocks }\end{array}$ & 1.5 & 1.7 & 1.5 & 1.1 & 1.5 \\
\hline Electricity, gas, and water supply & 1.2 & 1.5 & 0.7 & 0.5 & 1.5 \\
\hline Construction & 0.1 & 0.4 & 0.7 & 1.1 & 1.2 \\
\hline Wholesale and retail trade, and repair of vehicles and household goods & 1.3 & 1.7 & 1.4 & 2.5 & 3.6 \\
\hline Hotels and restaurants & 0.3 & 0.4 & 0.6 & 0.3 & 0.2 \\
\hline $\begin{array}{l}\text { Transport, storage, and communications } \\
\text { of which: }\end{array}$ & 1.1 & 3.2 & 3.5 & 2.3 & 1.6 \\
\hline Land & 0.8 & 2.7 & 2.8 & 1.4 & 1.0 \\
\hline Air & 0.0 & 0.1 & 0.2 & 0.1 & 0.1 \\
\hline Post and telecommunications & 0.2 & 0.1 & 0.3 & 0.1 & 0.1 \\
\hline Financial intermediation & 2.1 & 1.1 & 1.0 & 0.3 & 1.1 \\
\hline Real estate, renting, and business activities & 8.8 & 9.3 & 10.0 & 20.6 & 21.5 \\
\hline Public administration, education, health, and social work & 0.1 & 0.2 & 0.1 & 0.3 & 0.1 \\
\hline
\end{tabular}

Source: National Bank of Kazakhstan. 
Table 30. Kazakhstan: Stock of External Debt, 1999-2003

(In millions of U.S. dollars)

\begin{tabular}{|c|c|c|c|c|c|}
\hline & 1999 & 2000 & 2001 & 2002 & 2003 \\
\hline Total external debt & 12,089 & 12,684 & 15,158 & 18,221 & 22,859 \\
\hline Total public external debt & 4,057 & 3,929 & 3,800 & 3,501 & 3,631 \\
\hline IMF credit & 454 & 0 & 0 & 0 & 0 \\
\hline Government and government guaranteed debt & 3,602 & 3,929 & 3,800 & 3,501 & 3,631 \\
\hline Loans to the government & 2,896 & 3,578 & 3,577 & 2,944 & 3,073 \\
\hline Multilateral creditors & 1,472 & 1,508 & 1,549 & 1,568 & 1,544 \\
\hline World Bank & 1,106 & 1,122 & 1,164 & 1,175 & 1,143 \\
\hline EBRD & 49 & 45 & 41 & 36 & 36 \\
\hline ADB & 307 & 329 & 331 & 328 & 330 \\
\hline Islamic Development Bank & 10 & 11 & 13 & 29 & 35 \\
\hline Bilateral creditors $1 /$ & 774 & 776 & 713 & 435 & 601 \\
\hline Germany (KfW) & 5 & 7 & 9 & 17 & 21 \\
\hline Korea (EXIM bank) & 5 & 7 & 5 & 4 & 2 \\
\hline Japan (JEXIM) & 262 & 0 & 0 & 0 & 0 \\
\hline JBIC & 0 & 450 & 379 & 401 & 548 \\
\hline Austria & 4 & 3 & 3 & 3 & 3 \\
\hline Sweden & 3 & 3 & 3 & 3 & 3 \\
\hline $\mathrm{OECF} / \mathrm{JCB}$ & 191 & 0 & 0 & 0 & 0 \\
\hline Others & & & & 7 & 24 \\
\hline Foreign commercial banks and companies & 0 & 294 & 315 & 292 & 279 \\
\hline Eurobonds & 650 & 1,000 & 1,000 & 650 & 650 \\
\hline Loans guaranteed by the government (including medium- and long-term trade credits) & 707 & 351 & 223 & 557 & 558 \\
\hline Non-guaranteed external debt & 8,032 & 8,755 & 11,358 & 14,720 & 19,228 \\
\hline Intra-company loans & 6,162 & 6,931 & 8,879 & 10,709 & 12,029 \\
\hline Liabilities to unaffiliated creditors & 1,863 & 1,824 & 2,478 & 4,011 & 7,207 \\
\hline of which: Short-term & 1,678 & 814 & 951 & 1,637 & 2,628 \\
\hline \multicolumn{6}{|l|}{ Memorandum items: } \\
\hline \multicolumn{6}{|l|}{ Government and government guaranteed debt by creditor (in percent) } \\
\hline Multilateral creditors, excluding IMF & 41 & 38 & 41 & 45 & 43 \\
\hline Bilateral creditors & 22 & 20 & 19 & 21 & 24 \\
\hline Eurobonds & 18 & 25 & 26 & 19 & 18 \\
\hline Loans guaranteed by the government & 19 & 17 & 14 & 16 & 15 \\
\hline
\end{tabular}

Sources: Kazakhstani authorities; and Fund staff estimates.

1 / Numbers before 2002 include debt to commercial banks and companies and debt not covered elsewhere. 